\title{
Type-II quadrupole topological insulators
}

\author{
Yan-Bin Yang $\odot,{ }^{1} \mathrm{Kai} \mathrm{Li},{ }^{1}$ L.-M. Duan, ${ }^{1}$ and Yong Xu $\odot{ }^{1,2, *}$ \\ ${ }^{1}$ Center for Quantum Information, IIIS, Tsinghua University, Beijing 100084, People's Republic of China \\ ${ }^{2}$ Shanghai Qi Zhi Institute, Shanghai 200030, People's Republic of China
}

(Received 30 January 2020; revised 29 April 2020; accepted 16 June 2020; published 7 July 2020)

\begin{abstract}
Modern theory of electric polarization is formulated by the Berry phase, which, when quantized, leads to topological phases of matter. Such a formulation has recently been extended to higher electric multipole moments, through the discovery of the so-called quadupole topological insulator. It has been established by a classical electromagnetic theory that in a two-dimensional material the quantized properties for the quadupole topological insulator should satisfy a basic relation. Here we discover a new type of quadrupole topological insulator (dubbed type-II) that violates this relation due to the breakdown of the correspondence that a Wannier band and an edge energy spectrum close their gaps simultaneously. We find that, similarly to the previously discovered (referred to as type-I) quadrupole topological insulator, the type-II hosts topologically protected corner states carrying fractional corner charges. However, the edge polarizations only occur at a pair of boundaries in the type-II insulating phase, leading to the violation of the classical constraint. We demonstrate that such new topological phenomena can appear from quench dynamics in non-equilibrium systems, which can be experimentally observed in ultracold atomic gases. We also propose an experimental scheme with electric circuits to realize such a new topological phase of matter. The existence of the new topological insulating phase means that new multipole topological insulators with distinct properties can exist in broader contexts beyond classical constraints.
\end{abstract}

DOI: 10.1103/PhysRevResearch.2.033029

\section{INTRODUCTION}

Recently, the formulation of electric polarization based on the Berry phase has been extended to higher electric multipole moments, such as quadrupole moments and octupole moments $[1,2]$. Similarly to electric dipole moments, these multipole moments can be quantized due to crystalline symmetries, such as reflection symmetries, giving rise to multipole topological insulators. For a quadrupole topological insulator (QTI), besides the quantized quadrupole moment, the quantized edge polarization and fractional corner charge arise. Such fractional charges are associated with the appearance of the topologically protected corner states. The QTI has ignited an intensive study of higher-order topological insulators with $(n-m)$-dimensional edge states with $m>1$ for a $n$-dimensional system [1-23], in stark contrast to the conventional first-order topological insulators with $m=1$. Recently, the QTI has been experimentally observed [24-26].

For a two-dimensional (2D) square classical system with bulk quadrupole moments $q_{x y}$, a classical electromagnetic theory based on the multipole expansion of an electric potential shows that equal amplitude edge polarizations

\footnotetext{
*yongxuphy@tsinghua.edu.cn

Published by the American Physical Society under the terms of the Creative Commons Attribution 4.0 International license. Further distribution of this work must maintain attribution to the author(s) and the published article's title, journal citation, and DOI.
}

$p_{x, y}^{\text {edge } \alpha}$ and corner charges $Q^{\text {corner } \alpha, \beta}$ can be induced so that $\left|q_{x y}\right|=\left|p_{x}^{\text {edge } \pm y}\right|=\left|p_{y}^{\text {edge } \pm x}\right|=\left|Q^{\text {corner } \pm x, \pm y}\right|[1,2]$. Here, $p_{x}^{\text {edge } \beta}\left(p_{y}^{\text {edge } \alpha}\right)$ describes the edge polarization per unit length along the $x(y)$ direction at the $y$-normal (x-normal) boundaries. The edges perpendicular to $y(x)$ are labeled by the Greek letters $\beta= \pm y(\alpha= \pm x)$ with the sign denoting their relative positions. The currently discovered quantum QTIs indeed respect these relations [1,2] [see Fig. 1(a)]. Provided that a system has bulk-independent boundary dipole moments besides the bulk quadrupole moments, the relation is summarized as $Q^{\text {corner }+x,+y}=p_{y}^{\text {edge }+x}+p_{x}^{\text {edge }+y}-q_{x y}$. Previous research [27] has shown that a Wannier band and an edge energy spectrum should close their gaps simultaneously, giving rise to edge polarizations of equal amplitude at the $x$ normal and $y$-normal boundaries. The result is consistent with a previously established theory [28]. This simultaneous gap closing ensures that the classical relation has to be respected.

However, we find that the vanishing of the gap of the Wannier band is not necessarily associated with the vanishing of the gap of the edge energy spectrum. As a result, the edge polarization along one direction changes while that along the other direction remains, leading to a type of quadrupole topological insulating phase where $q_{x y}=$ $\left|Q^{\text {corner } \pm x, \pm y}\right|=\left|p_{x}^{\text {edge } \pm y}\right|=e / 2$, but $p_{y}^{\text {edge } \pm x}=0$, which is not equal to $p_{x}^{\text {edge } \pm y}$, violating the classical relation [see Fig. 1(b)]. It is worthwhile to note that the type-II quadrupole insulating phase is fundamentally different from the insulator with pure edge polarizations along one direction but without bulk quadrupole moments, where the classical relation 

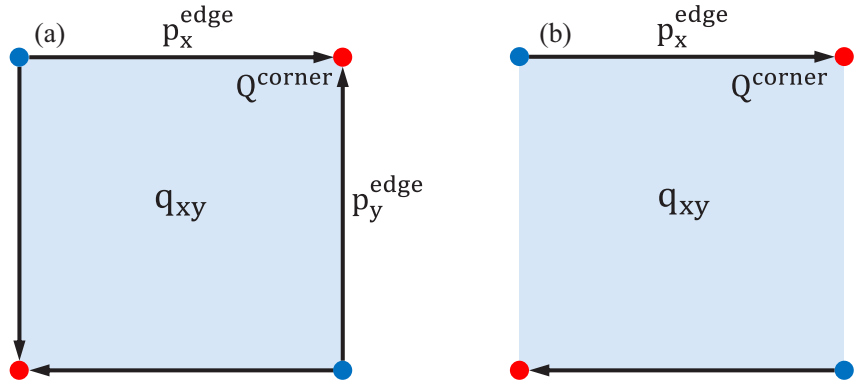

FIG. 1. Schematics of edge polarizations and corner charges. (a) Edge dipole moments exist at all boundaries in type-I QTI and (b) they exist only at the boundaries perpendicular to $y$ in type-II QTIs. Corner charges $Q^{\text {corner }}= \pm e / 2$ (marked by different colors) appear in both phases.

is also respected. It is also fundamentally different from a topologically trivial bulk insulator attached with a pair of Su-Schrieffer-Heeger (SSH) models. In this case, the bulk is trivial with zero quadrupole moments while the type-II quadrupole insulator exhibits nonzero quadrupole moments in the bulk as detailed in Appendix A.

Furthermore, we find anomalous quadrupole topological phases which have the zero Berry phase of the Wannier bands (referred to as Wannier-sector polarization) but the nonzero edge polarization. This tells us that the previously introduced nested Wilson loop formalism [1,2] cannot be used to characterize these insulating phases. Such phases arise because the Wannier Hamiltonian is fundamentally different from a static system Hamiltonian, given that the energy spectrum of the former is periodic, reminiscent of that of the effective Hamiltonian in a periodically driven system [16]. This allows the Wannier bands to close their gaps at either $v=0$ or $v= \pm 1 / 2$. When the Wannier spectrum under open boundary conditions exhibit both edge states at $v=0, \pm 1 / 2$, the Berry phase vanishes; this resembles a periodically driven system, where although the traditional topological invariant of a Hamiltonian vanishes, the edge state persists [29]. While such anomalous phenomena have also been found in a model with eight bands, the Berry phase can still be used to characterize the edge polarization [16]. But that does not work for our minimal quadrupole model. Here we introduce a new topological invariant for a Wilson line to characterize the edge polarization. The Wannier gap closing at either $v=0$ or $v= \pm 1 / 2$ can be reflected by the change of the topological invariants.

While we demonstrate the existence of the type-II QTI based on a particular model, we further show from a general perspective that it generically occurs in systems with reflection symmetries and the particle-hole or chiral symmetry in the presence of long-range hopping; such long-range hopping widely exists in various systems, such as photonic systems [30,31], polar molecules and Rydberg atoms [32-36], Shiba lattices [37-40], and shaken lattices where the effective hopping strength ratio can be tuned in a wide range [41-43]. Particularly, a new type of corner modes has recently been observed in a photonic kagome crystals with long-range interactions [31]. Based on this understanding, we further construct significantly simplified models that support the
type-II QTI. Furthermore, the breakdown of the correspondence between the Wannier spectrum gap and the edge spectrum gap suggests that the Wannier band alone can induce topological phase transitions despite the absence of the energy band gap closing. Indeed, we find another new topological phase with quantized edge polarizations but without zeroenergy corner modes and quadrupole moments. This transition arises from the relevant Wannier gap closing characterized by the change of the winding number for a Wilson line.

Similarly to the Chern insulator that exhibits a winding of the Berry phase, Ref. [1] introduces a three-dimensional higher-order topological insulator by breaking the reflection symmetries so that the quadrupole moment, edge polarizations at all boundaries and corner charges all exhibit a winding, which is associated with the presence of chiral hinge modes. Analogously, based on the type-II QTI, we find two types of new three-dimensional higher-order topological insulators. In one insulator, the winding of the quadrupole moment, corner charges and edge polarizations at a pair of boundaries appears associated with chiral hinge modes. In the other one, only the winding for the edge polarizations at a pair of boundaries happens.

Nonequilibrium dynamics under unitary time evolution between distinct topological phases have been studied in cold atoms from various aspects [44-60]. Given that the unitary evolution does not change the energy spectra of a parent Hamiltonian [56], the topology of the evolving states remains unchanged if symmetries of the parent Hamiltonian are preserved during unitary time evolution $[44,45,56]$. Specifically, let us start with a ground state $\left|\psi_{\mathbf{k}}\right\rangle$ of an initial Hamiltonian $H_{i}(\mathbf{k})$ and then suddenly change the Hamiltonian to $H_{f}(\mathbf{k})$ by tuning system parameters. The state then evolves under the final Hamiltonian, i.e., $\left|\psi_{\mathbf{k}}(t)\right\rangle=e^{-i H_{f}(\mathbf{k}) t}\left|\psi_{\mathbf{k}}\right\rangle$. Since the evolving state is an eigenstate of a parent Hamiltonian $H_{p}(\mathbf{k})=e^{-i H_{f}(\mathbf{k}) t} H_{i}(\mathbf{k}) e^{i H_{f}(\mathbf{k}) t}$, the topological properties of the evolving states are dictated by the parent Hamiltonian. If relevant symmetries of $H_{p}$ do not change as time progresses, then it has been shown that $H_{p}$ and $H_{i}$ share the same topological property given that the bulk energy gap of $H_{p}$ remains the same as $H_{i}$ during the time evolution [56]. On the other hand, a physical quantity, such as the Berry phase, can change continuously if symmetries of the parent Hamiltonian are allowed to change during unitary time evolution $[57,58]$. We here perform an investigation of the quench dynamics across distinct phases in the Benalcazar-Bernevig-Hughes (BBH) model and find that the type-II QTI phase emerges as time evolves, even though the symmetries are preserved during the time evolution. This shows that new topological phases can arise due to the Wannier gap closing in nonequilibrim dynamics.

The paper is organized as follows. In Sec. II, we demonstrate the existence of the type-II anomalous QTI (AQTI) by studying the topological properties of a tight-binding model. In Sec. III, we introduce a topological invariant for a Wilson line to characterize the edge polarization change due to the Wannier band gap closing and bulk energy gap closing. In Sec. IV, we provide a general analysis for the existence of the type-II QTI and show that the type-II phase generically appears in systems with reflection symmetries and the particle-hole or chiral symmetry in the presence of long-range 
hopping. Based on this analysis, we construct several significantly simplified models supporting the type-II QTI. We also present another topological phase with quantized edge polarizations but without zero-energy corner modes and quadrupole moments. In Sec. $\mathrm{V}$, we study the pumping phenomenon and three-dimensional higher-order topological insulators. In Sec. VI, we show that the type-II QTI can appear in quench dynamics through unitary time evolution. In Sec. VII, we demonstrate that the quench dynamics can be experimentally realized in cold atoms and further propose an experimental scheme using electric circuits to realize these new topological phenomena. Finally, the conclusion is presented in Sec. VIII.

\section{THE TYPE-II QTI}

To generate the type-II quadrupole topological insulating phase, we consider a 2D crystal with four sites in each unit cell and long-range hopping between unit cells. We enforce two reflection symmetries $M_{x}: x \rightarrow-x$ and $M_{y}: y \rightarrow-y$, in order to maintain the quantization of the quadrupole moment, corner charges and edge polarizations. Specifically, the system is described by the following Hamiltonian

$$
H=\sum_{\mathbf{R}} \sum_{d_{x}, d_{y}} \hat{c}_{\mathbf{R}+d_{x} \mathbf{e}_{x}+d_{y} \mathbf{e}_{y}} h_{\left(d_{x} d_{y}\right)} \hat{c}_{\mathbf{R}},
$$

where $\hat{c}_{\mathbf{R}}^{\dagger}=\left(\begin{array}{llll}\hat{c}_{\mathbf{R}, 1}^{\dagger} & \hat{c}_{\mathbf{R}, 2}^{\dagger} & \hat{c}_{\mathbf{R}, 3}^{\dagger} & \hat{c}_{\mathbf{R}, 4}^{\dagger}\end{array}\right)$ with $\hat{c}_{\mathbf{R}, \alpha}^{\dagger}\left(\hat{c}_{\mathbf{R}, \alpha}\right)$ creating (annihilating) an electron at a sublattice denoted by the index $\alpha=1,2,3,4$ within a unit cell denoted by a lattice vector $\mathbf{R}=R_{x} \mathbf{e}_{x}+R_{y} \mathbf{e}_{y}$ with $R_{x}$ and $R_{y}$ being integers. The sum over $d_{x}$ and $d_{y}$ depicts the on-site potential and the electron tunneling between distinct sublattices within a unit cell when $d_{x}=d_{y}=0$ and the tunneling between neighboring unit cells otherwise. For simplicity, we choose the lattice constant $a_{x, y}=1$. Here, we consider the case with long-range hopping including up to the hopping with $\left(d_{x}= \pm 1, d_{y}= \pm 2\right)$ and $\left(d_{x}= \pm 2, d_{y}= \pm 1\right)$. To be specific, we choose $h_{(00)}=\gamma\left(\tau_{1} \sigma_{0}+\tau_{2} \sigma_{2}\right)+\Delta \tau_{3} \sigma_{2}+\delta \tau_{3} \sigma_{0}$, $h_{(10)}=t_{1}\left(\tau_{1} \sigma_{0}-i \tau_{2} \sigma_{3}\right)+t_{1}^{\prime} \tau_{3} \sigma_{2}, h_{(01)}=-i t_{1} \tau_{2} \sigma_{-}+t_{1}^{\prime} \tau_{1} \sigma_{0}$, $h_{(11)}=t_{2}\left(-i \tau_{0} \sigma_{3}-i \tau_{3} \sigma_{-}+\tau_{1} \sigma_{0}-i \tau_{2} \sigma_{3}\right)-i t_{2}^{\prime} \tau_{2} \sigma_{-}, h_{(20)}=$ $t_{2}\left(-i \tau_{3} \sigma_{3}+i \tau_{0} \sigma_{1}+\tau_{3} \sigma_{2}\right), h_{(02)}=-i t_{2} \tau_{2} \sigma_{-}-i t_{2}^{\prime} \tau_{3} \sigma_{-}$, $h_{(21)}=t_{2}^{\prime}\left(-\tau_{1} \sigma_{0}+i \tau_{2} \sigma_{3}\right)$, and $h_{(12)}=i t_{2}^{\prime} \tau_{2} \sigma_{-}$, where $\sigma, \tau$ denote Pauli matrices for the degrees of freedom within a unit cell and $\sigma_{ \pm}=\sigma_{1} \pm i \sigma_{2}$. The other tunneling matrices for $d_{x} \neq 0$ and $d_{y} \neq 0$ can be obtained by $h_{\left(-d_{x}-d_{y}\right)}=\left(h_{\left(d_{x} d_{y}\right)}\right)^{\dagger}$, $\hat{m}_{x} h_{\left(d_{x} d_{y}\right)} \hat{m}_{x}^{\dagger}=h_{\left(-d_{x} d_{y}\right)}$, and $\hat{m}_{y} h_{\left(d_{x} d_{y}\right)} \hat{m}_{y}^{\dagger}=h_{\left(d_{x}-d_{y}\right)}$ required by the Hermiticity and reflection symmetries of the system with $\hat{m}_{x}=\tau_{1} \sigma_{3}$ and $\hat{m}_{y}=\tau_{1} \sigma_{1}$ for zero $\delta$. Specifically, we set $\Delta=$ $t_{1}=0.3, t_{1}^{\prime}=0.2, t_{2}=0.15$, and $t_{2}^{\prime}=0.1$. The Hamiltonian in momentum space can be found in Appendix B. When $\delta=$ 0 , this Hamiltonian also respects the particle-hole symmetry to protect the zero-energy corner modes, i.e., $\mathrm{CHC}^{-1}=H$ with $\mathcal{C} \hat{c}_{\mathbf{R}, \alpha} \mathcal{C}^{-1}=\sum_{\beta=1}^{4}\left(\tau_{3} \sigma_{0}\right)_{\alpha \beta} \hat{c}_{\mathbf{R}, \beta}^{\dagger}$. The expression for the particle-hole symmetry in momentum space can be found in Appendix B. Compared with the model in Ref. [1], our model does not preserve the time-reversal symmetry $\Theta=\kappa(\kappa$ is the complex conjugation operator) and thus the system does not have the $P T=\hat{m}_{x} \hat{m}_{y} \kappa$ symmetry, which guarantees the double degeneracy of the energy bands. Our model breaks this symmetry, lifting the energy degeneracy.

Our numerical computation shows the presence of an energy gap in momentum space energy spectra with respect to $\gamma$ unless $\gamma=-0.69$ and $\gamma=0.61$, where the energy gap vanishes. This implies that the bulk of the system exhibits the insulating property in the gapped regions at half filling. The insulating feature can also be seen in the energy spectra under open boundary conditions along both $x$ and $y$ directions [see Fig. 2(c)]. However, for $-0.69<\gamma<0.34$ and $0.61<\gamma<$ 1.03, imposing open boundaries render the appearance of four zero-energy states localized at the corners corresponding to a second-order topological insulator, where corner states exist at the boundaries of boundaries as shown in Fig. 2(d). In other parameter regions, we do not find zero-energy corner states.

These corner states give rise to fractional charges $\pm e / 2$ localized at the corners. Such corner charges are numerically calculated by performing the integration of the charge density over a quadrant of the system [2],

$$
Q^{\text {corner }-x,-y}=\sum_{R_{x}=1}^{N_{x} / 2} \sum_{R_{y}=1}^{N_{y} / 2} \rho(\mathbf{R}),
$$

where $\rho(\mathbf{R})=2 e-e \sum_{n=1}^{N_{\text {occ }}} \sum_{\alpha=1}^{4}\left|\left[u^{n}\right]^{\mathbf{R}, \alpha}\right|^{2}$ is the charge density with the first term contributed by the atomic positive charges and the second term by the electron distribution described by the $n$th occupied eigenstate $\left|u^{n}\right\rangle$ of our Hamiltonian under open boundary conditions with $\left[u^{n}\right]^{\mathbf{R}, \alpha}$ being the component at the site $\mathbf{R}$ with orbital index $\alpha$. To calculate the corner charge, we include a small $\delta$ so that the fourfold degeneracy of the zero-energy states is lifted, leading to two corner states with positive energy and the other two with negative energy. At half filling, only two corner states are occupied. Suppose the atoms contribute $+2 e$ charge in each unit cell. This gives us the corner-localized fractional charges $\pm e / 2$ in the limit $\delta \rightarrow 0$.

To show that the insulator with zero-energy corner states is a QTI, we calculate their quadrupole moments based on the following formula $[61,62]$

$$
q_{x y}=\frac{1}{2 \pi} \operatorname{Im}\left[\log \left\langle\Psi_{G}\left|\hat{U}_{2}\right| \Psi_{G}\right\rangle\right]
$$

where $\hat{U}_{2}=e^{2 \pi i \sum_{\mathbf{r}} \hat{q}_{x y}(\mathbf{r})}$ with $\hat{q}_{x y}(\mathbf{r})=x y \hat{n}(\mathbf{r}) /\left(L_{x} L_{y}\right)$ being the quadrupole moment per unit cell measured with respect to $x=y=0$ at the site $\mathbf{r}, L_{x}$ and $L_{y}$ are the length of the system along $x$ and $y$ directions, respectively, the sum is over $(x, y) \in\left(0, L_{x}\right] \times\left(0, L_{y}\right], \hat{n}(\mathbf{r})$ is the number of electrons at the site $\mathbf{r}$ and $\left|\Psi_{G}\right\rangle$ is the many-body ground state of a system. Our calculation is performed under periodic boundary conditions. Note that the atomic positive charge contribution has been deducted.

Our numerical results show that the system has a quantized quadrupole moment $q_{x y}=e / 2$ (protected by the reflection symmetry) in the region where the zero-energy corner modes exist, as shown in Fig. 2(b). The change of the quadrupole moment is associated with the vanishing of either a bulk energy gap or an edge energy gap, reflecting the topological properties of the quadrupole insulating phase. We note that while Ref. [63] points out some difficulties for evaluating the 
(a)

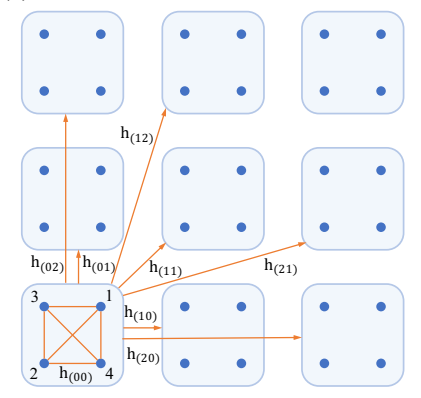

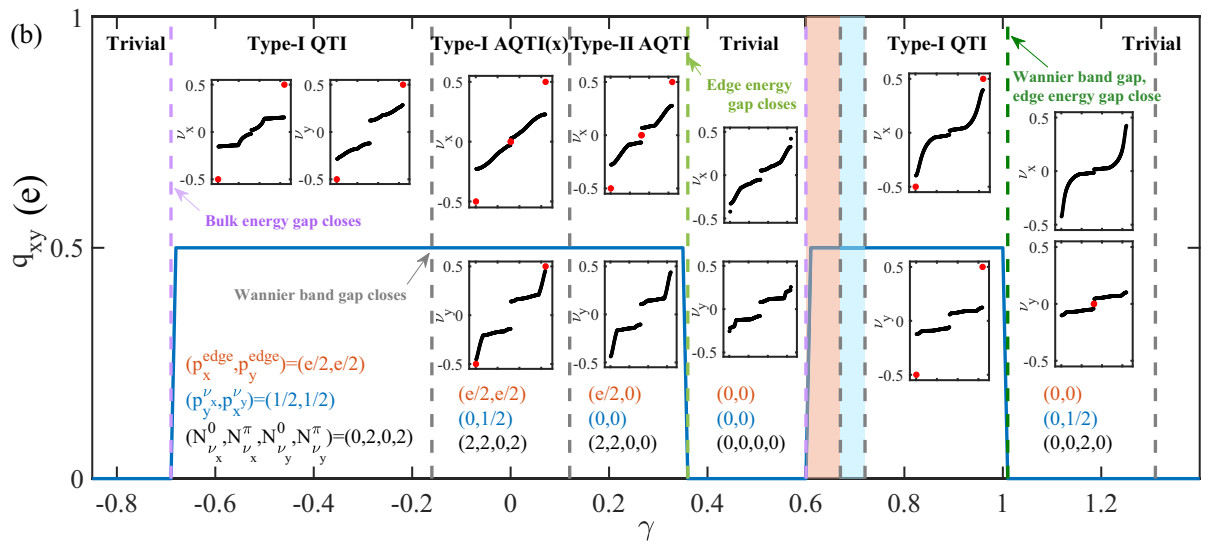

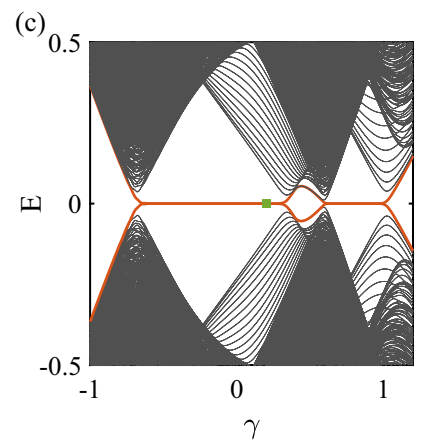

(d)
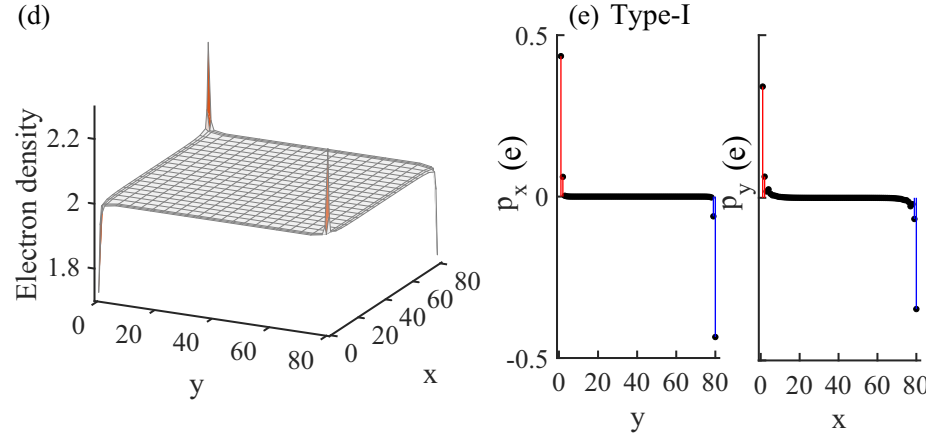

(f) Type-II

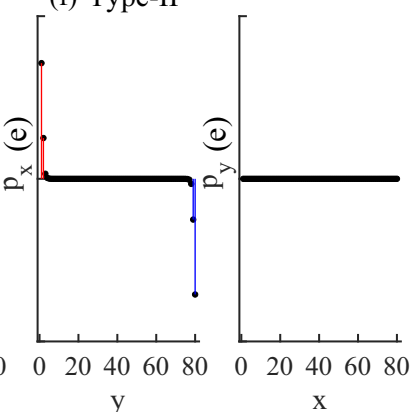

FIG. 2. Schematics of our model, phase diagram and topological properties. (a) Schematics of the tunneling in our tight-binding model. (b) Phase diagram with respect to a system parameter $\gamma$. The quadrupole moment evaluated for a $80 \times 80$ system is plotted as a blue line. In the phase diagram, we observe the topologically trivial insulator, the type-I QTI, the type-I anomalous quadrupole topological insulator (AQTI) (anomalousness exists in the Wannier band $v_{x}$ ) and the type-II AQTI. The subsets display the Wannier spectrum $v_{x}\left(v_{y}\right)$ in a cylinder geometry with periodic boundaries along $x(y)$ and open ones along $y(x)$ with the isolated Wannier centers highlighted by red circles. The edge polarization ( $p_{x}^{\text {edge }}, p_{y}^{\text {edge }}$ ) calculated using the formula (6), the Wannier-sector polarization $\left(p_{y}^{v_{x}}, p_{x}^{v_{y}}\right)$, the number of edge states of the Wannier Hamiltonian $N_{v} \equiv\left(N_{v_{x}}^{0}, N_{v_{x}}^{\pi}, N_{v_{y}}^{0}, N_{v_{y}}^{\pi}\right)$ are also shown. The vertical dashed lines represent the critical points where the bulk energy gap, the edge energy gap, or the Wannier spectrum gap vanish. The light red and blue regions denote the type-I AQTI(xy) (anomalousness exists in both Wannier band $v_{x}$ and $v_{y}$ ) and type-I AQTI(x). Richer phase diagram for the topologically trivial phase can be found in Appendix C. (c) The energy spectrum as a function of $\gamma$ for open boundary conditions along both $x$ and $y$ directions with zero-energy corner modes being highlighted by a red line. (d) The electron density distribution in a typical type-II AQTI phase with the zero-energy corner modes marked by the green square in (c). Here, a very small $\delta$ is imposed so that two corner states are occupied. [(e) and (f)] The edge polarization profiles for a type-I AQTI state at $\gamma=-0.1$ and a type-II AQTI state at $\gamma=0.2$, respectively.

quadrupole moment in a generic system using the formula proposed in Ref. [61,62], the calculated quadrupole moments in our model are reasonable as verified in Appendix A.

To characterize the edge polarization (for example, the polarization along $x$ ), we consider the Wilson loop

$$
\mathcal{W}_{x}=F_{x, k_{x}+\left(N_{x}-1\right) \delta k_{x}} \cdots F_{x, k_{x}}
$$

and similarly for $\mathcal{W}_{y}$, where the subscript $x(y)$ indicates that the Wilson loop is defined following the path along $x(y)$. Here $\left[F_{x, k_{x}}\right]^{m n}=\left\langle u_{k_{x}+\delta k_{x}}^{m} \mid u_{k_{x}}^{n}\right\rangle$, where $\delta k_{x}=2 \pi / N_{x}$ with $N_{x}$ being the number of unit cells along $x$, and $k_{x}$ is the quasimomentum along $x$ due to the imposed periodic boundary condition along that direction $[1,2]$. For a system with periodic boundaries along $y,\left|u_{k_{x}}^{n}\right\rangle=\left|u_{k_{x}, k_{y}}^{n}\right\rangle$ refers to the occupied eigenstate of a system Hamiltonian in momentum space with $n$ being the band index. Yet, for a system with open boundaries, $\left|u_{k_{x}}^{n}\right\rangle$ refers to the occupied eigenstate of the Hamiltonian with open boundaries along $y$. The Wannier Hamiltonian $H_{\mathcal{W}_{x}}$ is defined by $\mathcal{W}_{x} \equiv e^{i H_{\mathcal{W}_{x}}}$ with its eigenvalues $2 \pi v_{x}$ referred to as the Wannier spectrum, where $v_{x}$ is the Wannier center that determines the polarization that each state contributes. Here the reflection symmetry maintains the vanishing of the total polarization in the bulk [1,2]. Let us first consider a cylinder geometry with open boundaries along $y$. In this case, when the Wannier spectrum exhibits isolated eigenvalues at $v_{x}= \pm 1 / 2$, with the corresponding eigenstates being localized at two opposite $y$-normal boundaries, the system has the boundary polarization $p_{x}^{\text {edge }}$. The appearance of the edge polarization stems from the topological property of the bulk. There are two routes to the emergence of the edge states in the Wannier spectrum. One is through the change of the topological property of the Wannier bands, the eigenvalues $v_{x}\left(k_{y}\right)$ of $H_{\mathcal{W}_{x}}\left(k_{y}\right)$, under periodic boundary conditions along $y$, by closing the Wannier band gap at $v_{x}= \pm 1 / 2$. An alternative route is provided by closing either the bulk energy gap or edge energy gap, resulting in an abrupt change of the quadrupole moment.

To distinguish between the type-I and type-II QTIs, it is necessary to characterize their edge polarization by the Wilson loop. In a torus geometry, the eigenvalues of the Wilson loop $\mathcal{W}_{x}\left(k_{y}\right)$ [similarly for $\mathcal{W}_{y}\left(k_{x}\right)$ ] takes the form of $e^{i 2 \pi v_{x}^{j}\left(k_{y}\right)}$ 
with $j=1, \ldots, N_{\text {occ }}$ and $N_{\text {occ }}$ being the number of occupied bands since $\mathcal{W}_{x}\left(k_{y}\right)$ is unitary, implying that $H_{\mathcal{W}_{x}}\left(k_{y}\right)$ has eigenvalues $2 \pi v_{x}^{j}\left(k_{y}\right)$. Because $e^{i 2 \pi v_{x}^{j}\left(k_{y}\right)}$ repeats over intervals of 1 for $v_{x}^{j}\left(k_{y}\right)$, we restrict $v_{x}^{j}\left(k_{y}\right)$ to $(-0.5,0.5]$. Because of the reflection symmetry $M_{x}: x \rightarrow-x, \pm 2 \pi v_{x}^{j}\left(k_{y}\right)$ are both eigenvalues of $H_{\mathcal{W}_{x}}\left(k_{y}\right)$, so that the Wannier centers appear in pairs $\left[-v_{x}^{j}\left(k_{y}\right), v_{x}^{j}\left(k_{y}\right)\right]$, maintaining the vanishing of the bulk dipole moments in our model. The Wannier bands can be gapped with one band $v_{x}^{-}\left(k_{y}\right) \in(-0.5,0)$ and the other $v_{x}^{+}\left(k_{y}\right) \in(0,0.5)$ similarly to a conventional band. However, it turns out that there are two gaps for the Wannier bands: one is around $v_{x}=0$ and the other around $v_{x}= \pm 1 / 2$; the gaps can close at either $v_{x}=0$ or $v_{x}= \pm 1 / 2$.

Figure 2 illustrates that in the type-I phase, both Wannier spectra $v_{x}$ and $v_{y}$ under corresponding open boundary conditions (open along $y$ and $x$, respectively) exhibit isolated eigenvalues at $v_{x}= \pm 1 / 2$, which disappear under periodic boundary conditions, implying that they are contributed by the boundary states. Their emergence indicates the presence of the dipole moments at all the four boundaries. Remarkably, in the type-II phase, only $v_{x}= \pm 1 / 2$ occurs but not for $v_{y}$, implying that the dipole moments only exist at the $y$-normal edge but not at the $x$-normal one, as shown in Fig. 1(b).

To show that the dipole moments are localized at boundaries, we calculate the polarization distribution by

$$
p_{x}\left(R_{y}\right)=\sum_{j= \pm} \rho^{j}\left(R_{y}\right) v_{x}^{j}
$$

where $\rho^{j}\left(R_{y}\right)=\frac{1}{N_{x}} \sum_{k_{x}, \alpha}\left|\sum_{n=1}^{N_{\text {occ }}}\left[u_{k_{x}}^{n}\right]^{R_{y}, \alpha}\left[v_{k_{x}}^{j}\right]^{n}\right|^{2}$ is the probability density of the hybrid Wannier functions [1,2], $\left[v_{k_{x}}^{j}\right]^{n}$ is the $n$th entry of the $j$ th eigenvector $\left|v_{k_{x}}^{j}\right\rangle$ of the Wannier Hamiltonian corresponding to the Wannier center $v_{x}^{j}$ in a cylinder geometry with open boundaries along $y$, and $\left[u_{k_{x}}^{n}\right]^{R_{y}, \alpha}$ describes the collection of entries of the $n$th occupied eigenstate of our Hamiltonian in the same boundary configuration. The edge polarization is defined as the sum of $p_{x}\left(R_{y}\right)$ over a half along $y$, i.e.,

$$
\begin{aligned}
p_{x}^{\text {edge }-y} & =\sum_{R_{y}=1}^{N_{y} / 2} p_{x}\left(R_{y}\right) \\
& =-p_{x}^{\text {edge }+y}=-\sum_{R_{y}=N_{y} / 2+1}^{N_{y}} p_{x}\left(R_{y}\right),
\end{aligned}
$$

which is quantized. The formulation of the edge polarization along $y$ is similar.

Figures 2(e) and 2(f) show that the polarization, if exists, is indeed exponentially localized at the boundaries and opposite boundaries have opposite polarizations. While the polarization has a distribution along the direction perpendicular to a boundary, their total value for an edge is quantized, i.e., $p_{x, y}^{\text {edge }}= \pm e / 2$. Despite the presence of the edge dipole moments, the total polarization vanishes as opposite boundaries have opposite edge polarizations. In the type-II phase, the polarization along $y$ remains zero, in stark contrast to the corresponding nonzero edge polarization in the type-I phase.
Although the Wannier Hamiltonian can have the edge states at $v_{x}= \pm 1 / 2$ or $v_{x}=0$, only the former contributes to the edge polarization. In fact, both of these states at $v_{x}=$ $\pm 1 / 2$ or $v_{x}=0$ can appear simultaneously. In that case, we will show that the Wannier-sector polarization is zero and thus cannot be used to characterize these edge states. We refer to such a topological insulating phase as an anomalous QTI.

The number of the edge states of the Wannier Hamiltonian $H_{\mathcal{W}_{x}}\left(H_{\mathcal{W}_{y}}\right)$ changes when the gap of the bulk energy spectrum $E\left(k_{x}, k_{y}\right)$, edge energy spectrum $E^{\text {edge, } y}\left(k_{x}\right)$ $\left[E^{\text {edge, } x}\left(k_{y}\right)\right]$ at the $y$-normal $(x$-normal) edges or Wannier band $v_{x}\left(k_{y}\right)\left[v_{y}\left(k_{x}\right)\right]$ closes, reflecting the topological feature of the edge polarization. Associated with the vanishing of the bulk energy gap or edge energy gap is the change of the number of the edge states of the Wannier Hamiltonian corresponding to both $v_{x}=0$ and $v_{x}= \pm 1 / 2$. However, when the Wannier bands close their gap at $v_{x}=0$ or $v_{x}= \pm 1 / 2$, only the number of the edge states with the same eigenvalue as that where the gap vanishes changes. Specifically, the bulk energy gap closes at $\gamma=-0.69$ and $\gamma=0.61$, leading to the phase transition between the topologically trivial phase with $N_{v}=(2,0,2,0)$ and type-I quadrupole insulating phase with $N_{v}=(0,2,0,2)$, and the transition between a trivial phase with $N_{v}=(0,0,0,0)$ and the type-I anomalous phase with $N_{v}=(2,2,2,2)$, respectively. Here, $N_{v} \equiv\left(N_{v_{x}}^{0}, N_{v_{x}}^{\pi}, N_{v_{y}}^{0}, N_{v_{y}}^{\pi}\right)$ with $N_{v_{\lambda}}^{\epsilon}(\lambda=x, y)$ denoting the number of the edge sates of the Wannier Hamiltonian $\mathcal{W}_{\lambda}$ corresponding to the eigenvalue $\epsilon=0, \pi$. The vanishing gap of the Wannier bands divides the quadrupole insulating phase for $-0.69<\gamma<0.34$ into three regions: type-I QTI, type-I AQTI, and type-II AQTI; each gap closure gives rise to the change of the number of the corresponding edge states, as shown in Fig. 2(b). The edge energy spectrum at the $y$-normal boundary closes its gap at $\gamma=0.34$, resulting in the phase transition between a trivial phase with $N_{v}=(0,0,0,0)$ and the type-II AQTI with $N_{v}=(2,2,0,0)$

Our results show that the Wannier bands $v_{x}\left(k_{y}\right)\left[v_{y}\left(k_{x}\right)\right]$ do not necessarily close their gap at $v= \pm 1 / 2$ at the same time as the edge energy spectrum localized at the $x$-normal ( $y$-normal) boundaries (see Sec. IV for details). If their gaps always vanish simultaneously, there should be equal number of the edge states of the Wannier Hamiltonian $\mathcal{W}_{x}$ and $\mathcal{W}_{y}$ with eigenvalues $v= \pm 1 / 2$, giving rise to the same amplitude edge polarization at the $x$-normal and $y$-normal boundaries [see the case for $\gamma=1.03$ in Fig. 2(b)] [27]. With this violation in our model, we find the type-II phase where the dipole moments only exist at the boundaries vertical to $y$.

\section{A TOPOLOGICAL INVARIANT FOR A WILSON LINE}

The Wannier-sector polarization for the Wannier band $v_{x}^{ \pm}$ (similarly for $v_{y}^{ \pm}$) is defined as $[1,2]$

$$
p_{y}^{v_{x}^{ \pm}}=-\frac{1}{(2 \pi)^{2}} \int_{B Z} d^{2} \mathbf{k} \mathcal{A}_{y, \mathbf{k}}^{ \pm},
$$

where $\mathcal{A}_{y, \mathbf{k}}=-i\left\langle w_{x, \mathbf{k}}^{ \pm}\left|\partial_{k_{y}}\right| w_{x, \mathbf{k}}^{ \pm}\right\rangle$is the Berry connection over the Wannier bands $v_{x}^{ \pm}$, respectively. $\left|w_{x, \mathbf{k}}^{ \pm}\right\rangle=$ $\sum_{n=1,2}\left|u_{\mathbf{k}}^{n}\right\rangle\left[v_{x, \mathbf{k}}^{ \pm}\right]^{n}$ with $\left|u_{\mathbf{k}}^{n}\right\rangle$ being the $n$th occupied eigenstate of our Hamiltonian in momentum space and $\left[v_{x, \mathbf{k}}^{ \pm}\right]^{n}$ being the 
$n$th entry of the eigenstate $\left|v_{x, \mathbf{k}}^{ \pm}\right\rangle$of the Wannier Hamiltonian in a torus geometry.

The Wannier-sector polarizations were previously introduced to characterize the edge polarizations of the type-I QTI. However, when it becomes anomalous, we find that a corresponding Wannier-sector polarization vanishes [see their values $\left(p_{y}^{v_{x}}, p_{x}^{v_{y}}\right)$ in Fig. 2(b)], suggesting that the Wanniersector polarization cannot uniquely identify the edge dipole moments.

To characterize the edge polarization along $x$ (similarly along $y$ ), we will introduce a topological invariant based on the Wilson line with respect to $\epsilon$ defined as

$$
\mathcal{W}_{k_{x} \leftarrow 0}^{\epsilon}\left(k_{y}\right) \equiv \mathcal{W}_{k_{x} \leftarrow 0}\left(k_{y}\right) e^{-i H_{W_{x}}^{\epsilon}\left(k_{y}\right) k_{x} /(2 \pi)},
$$

where $\mathcal{W}_{k_{x} \leftarrow 0}\left(k_{y}\right)=F_{x,\left(k_{x}, k_{y}\right)} F_{x,\left(k_{x}-\delta k_{x}, k_{y}\right)} \cdots F_{x,\left(0, k_{y}\right)}$ is the Wilson line and $H_{W_{x}}^{\epsilon}\left(k_{y}\right) \equiv-i \log _{\epsilon} \mathcal{W}_{2 \pi \leftarrow 0}\left(k_{y}\right)$ is the Wannier Hamiltonian with respect to $\epsilon$ with $\log _{\epsilon}\left(e^{i \phi}\right)=i \phi$ with $\epsilon \leqslant$ $\phi<\epsilon+2 \pi$. The reflection symmetry leads to (the details are presented in Appendix D):

$$
\begin{gathered}
S \mathcal{W}_{\pi \leftarrow 0}^{\epsilon=0}\left(k_{y}\right) S^{\dagger}=-\mathcal{W}_{\pi \leftarrow 0}^{\epsilon=0}\left(k_{y}\right), \\
S \mathcal{W}_{\pi \leftarrow 0}^{\epsilon=\pi}\left(k_{y}\right) S^{\dagger}=\mathcal{W}_{\pi \leftarrow 0}^{\epsilon=\pi}\left(k_{y}\right),
\end{gathered}
$$

where $S=\sigma_{z}$. In the basis consisting of eigenvectors of $S$,

$$
\mathcal{W}_{\pi \leftarrow 0}^{\epsilon=0}\left(k_{y}\right)=\left[\begin{array}{cc}
0 & U_{+}^{\epsilon=0}\left(k_{y}\right) \\
U_{-}^{\epsilon=0}\left(k_{y}\right) & 0
\end{array}\right]
$$

and

$$
\mathcal{W}_{\pi \leftarrow 0}^{\epsilon=\pi}\left(k_{y}\right)=\left[\begin{array}{cc}
U_{+}^{\epsilon=\pi}\left(k_{y}\right) & 0 \\
0 & U_{-}^{\epsilon=\pi}\left(k_{y}\right)
\end{array}\right] .
$$

Hence, we can define a winding number at $\epsilon=0, \pi$ as

$$
W_{v_{x}}^{\epsilon}=\frac{1}{2 \pi i} \int_{0}^{2 \pi} d k_{y} \partial_{k_{y}} \log U_{+}^{\epsilon}\left(k_{y}\right) .
$$

As presented in Appendix D, the winding number can change under a gauge transformation for the occupied eigenstates, since the Wilson line is defined by these eigenstates, in sharp contrast to the Floquet case, where the winding number is defined for an evolution operator [64,65]. It suggests that a definite physical quantity is the change of the winding number as a system parameter $\gamma$ varies. During this change, the Berry phase of the occupied bands should vary continuously, as discussed in Appendix D. Fortunately, the quantized dipole moment is a $Z_{2}$ quantity, so that the change of the winding number is sufficient to characterize the edge polarization.

The winding number changes as the bulk energy gap and Wannier band gap close, but does not respond to the closure of the edge energy gap, which is reasonable as the Wannier bands are constructed from the wave functions without any edge. However, the closure of the edge energy gap is associated with the change of the quadrupole moment. Taking into account the edge energy gap closure, we define

$$
\begin{aligned}
p_{x}^{\text {edge }}\left(\gamma_{1}\right)-p_{x}^{\text {edge }}\left(\gamma_{0}\right)= & e\left[\left(W_{\nu_{x}}^{\epsilon=\pi}\left(\gamma_{1}\right)-W_{v_{x}}^{\epsilon=\pi}\left(\gamma_{0}\right)\right.\right. \\
& \left.\left.-\Delta N_{q, x}\right) / 2\right] \bmod (1),
\end{aligned}
$$

where $\Delta N_{q, x}$ represents the number of times that the quadrupole moment changes due to the gap closure of the edge energy spectrum at the boundaries perpendicular to $y$,

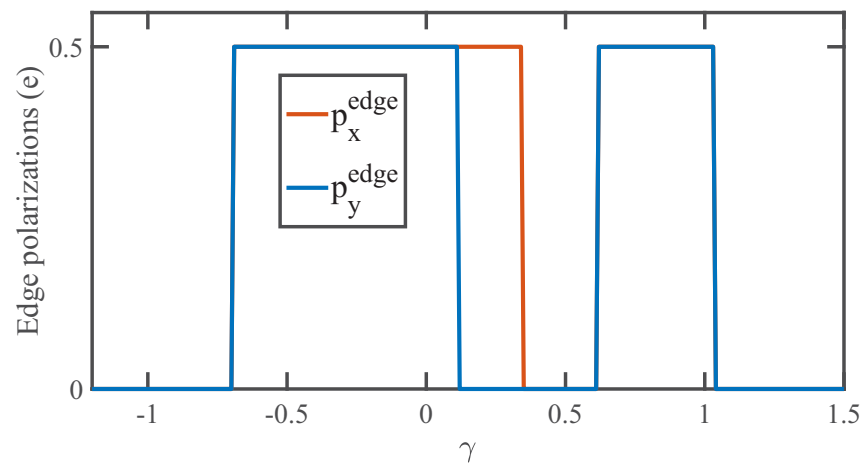

FIG. 3. The edge polarizations calculated using the formula (15) by choosing a gauge such that $W_{v_{x}}^{\epsilon=\pi}\left(\gamma_{0}=-1\right)=W_{v_{y}}^{\epsilon=\pi}\left(\gamma_{0}=\right.$ $-1)=0$. The edge polarization $p_{x}^{\text {edge }}$ and $p_{y}^{\text {edge }}$ in units of $e$ are plotted as the red and blue lines, respectively.

when we vary $\gamma$ from $\gamma_{0}$ to $\gamma_{1}$. This shows that the topology of the bulk spectrum dictates the edge polarization as the right sides are determined by the bulk property. In fact, $\Delta N_{q, x}$ is also associated with the number of times of the change of a parity (eigenvalue of $\hat{m}_{x}$ ) at the high-symmetric points $k_{x}=0$ or $k_{x}=\pi$ for a state localized at one boundary perpendicular to $y$ (see more detailed discussion in the following section). Provided that we start from a topologically trivial phase, i.e., $p_{x}^{\text {edge }}\left(\gamma_{0}\right)=0$, the formula can be reduced to

$$
p_{x}^{\text {edge }}\left(\gamma_{1}\right)=e\left[\left(W_{v_{x}}^{\epsilon=\pi}\left(\gamma_{1}\right)-\Delta N_{q, x}\right) / 2\right] \bmod (1)
$$

by choosing a gauge such that $W_{v_{x}}^{\epsilon=\pi}\left(\gamma_{0}\right)=0$.

In Fig. 3, we plot the edge polarizations $p_{x, y}^{\text {edge }}$ as a function of $\gamma$, calculated based on the formula (15) by choosing a gauge such that $W_{v_{x}}^{\epsilon=\pi}\left(\gamma_{0}=-1\right)=W_{v_{y}}^{\epsilon=\pi}\left(\gamma_{0}=-1\right)=0$ given that the phase is topologically trivial when $\gamma=-1$. The results are consistent with the Wannier spectrum in a cylinder geometry and the edge polarization calculated using the hybrid Wannier functions.

\section{INEQUIVALENCE BETWEEN WANNIER AND EDGE ENERGY SPECTRA DUE TO LONG-RANGE HOPPING}

\section{A. A general analysis}

As we have already discussed that the type-II QTI arises from the fact that the Wannier band and edge energy gaps do not vanish simultaneously. In this subsection, we will demonstrate from a general perspective that the breakdown occurs when the next-nearest-neighbor intercell hopping is appropriately included.

We now consider a generic four band Hamiltonian $H_{g}\left(k_{x}, k_{y}\right)$ in momentum space to describe a QTI. The Hamiltonian is required to respect two reflection symmetries $\hat{m}_{x}$ and $\hat{m}_{y}$ to maintain the vanishing of the bulk polarization as well as the quantization of quadrupole moments, corner charges and edge polarizations. In addition, either the particle-hole or chiral symmetry is enforced to ensure that the corner modes have zero energy. We note that the breakdown of the correspondence between Wannier and edge spectra was also found in a system with neither particle-hole nor chiral symmetry [66]. To have the gapped Wannier bands, we further require 
that $\hat{m}_{x}$ and $\hat{m}_{y}$ anticommute, and at each high-symmetric line, e.g., $k_{x}=k^{*}\left(k_{y}=k^{*}\right)$ with $k^{*}=0, \pi$, the eigenvalues of $\hat{m}_{x}\left(\hat{m}_{y}\right)$ of two occupied bands should occur in pairs as $(1,-1)[1,67]$. We also require that these parities for two unoccupied bands also occur in pairs as $(1,-1)$. Due to the reflection symmetry, we can write the Hamiltonian at the highsymmetric lines $k_{x}=k^{*}$ (similarly for $k_{y}=k^{*}$ ) as a direct sum of two submatrices in a basis $\beta=\beta_{1} \cup \beta_{-1}$ consisting of eigenvectors of $\hat{m}_{x}$,

$$
\left[H_{g}\left(k_{x}=k^{*}, k_{y}\right)\right]_{\beta}=H_{1}\left(k_{y}\right) \oplus H_{2}\left(k_{y}\right),
$$

where $H_{1}\left(k_{y}\right)$ and $H_{2}\left(k_{y}\right)$ are the matrix representation of $H_{g}$ with respect to the basis $\beta_{1}$ and $\beta_{-1}$, respectively. Here $\beta_{1}$ and $\beta_{-1}$ are the bases of the eigenspaces of $\hat{m}_{x}$ with the corresponding eigenvalues being 1 and -1 , respectively. In this basis, the representation of the reflection symmetry along $x$ can be chosen as $\hat{m}_{x}=\sigma_{z} \otimes \sigma_{0}$. For convenience, we use $\sigma$ instead of $\tau$ and explicitly write out the tensor product operation $\otimes$ throughout this section. To enforce the anticommutation relation for $\hat{m}_{x}$ and $\hat{m}_{y}$, i.e., $\left\{\hat{m}_{x}, \hat{m}_{y}\right\}=0$, we require $\hat{m}_{y}=\sigma_{\mu} \otimes \sigma_{\lambda}$ with $\mu=1,2$ and $\lambda=0,1,2,3$. Now we have $H_{2}\left(k_{y}\right)=\sigma_{\lambda} H_{1}\left(-k_{y}\right) \sigma_{\lambda}$. Clearly, the Berry phases of the occupied band of $H_{1}$ and $H_{2}$ take the opposite values. These Berry phases also determine the Wannier spectra $v_{y}\left(k_{x}\right)$ at $k_{x}=k^{*}$. In addition, the particle-hole or chiral symmetry forbids the existence of the terms $\sigma_{0}$ and $\cos k_{y} \sigma_{0}$ in $H_{1}$ but allows the existence of the term $\sin k_{y} \sigma_{0}$ in it.

We now discuss how the parity of an edge state localized at a boundary changes. Suppose that at a critical point, $H_{1}$ has zero-energy modes under open boundary conditions but its energy spectra under periodic boundary conditions remain gapped, implying the existence of the states localized at two boundaries. Away from this point where the gap is opened, only one edge state of $H_{1}$ is occupied, say, without loss of generality, the state localized at the top boundary. Then, due to the reflection symmetry along $y$, the occupied edge state of $H_{2}$ should be localized at the bottom boundary. Evidently, the parity of the state localized at the top (bottom) edge is 1 $(-1)$. When the edge energy gap for $H_{1}$ and $H_{2}$ closes and then reopens, if the position of the occupied edge state of $H_{1}$ changes from the top to the bottom, then the position of the occupied edge state of $\mathrm{H}_{2}$ changes from the bottom to the top. As a result, the parity of the state localized at the top (bottom) edge changes from $1(-1)$ to -1 (1), leading to the change of the edge polarization $p_{x}^{\text {edge }}$ and the quadrupole moment.

We are now in a position to ask two questions:

(1) If $C_{1}=\pi$, then does $H_{1}$ always exhibit zero-energy edge modes in a geometry with open boundaries?

(2) Conversely, if $H_{1}$ has zero-energy edge modes under open boundary conditions, then does $C_{1}=\pi$ always hold?

Here $C_{1}$ (modulo $2 \pi$ ) denotes the Berry phase of the occupied band of $H_{1}$.

If the answers to these questions are both yes, then the Wannier gap closing of $H_{g}$ at $v_{y}= \pm 1 / 2$ is always associated with the $y$-normal boundary spectra gap closing. For instance, provided that $H_{1}$ has either the particle-hole or chiral symmetry, it is a well-known fact that $H_{1}$ exhibits zero-energy edge modes under open boundary conditions if $C_{1}=\pi$.
To show that both of the two questions are answered yes for the BBH model [1], let us write the model in the following form:

$$
H_{\mathrm{BBH}}=\sigma_{0} \otimes H_{\mathrm{SSH}}\left(k_{y}, t_{y}\right)+H_{\mathrm{SSH}}\left(k_{x}, t_{x}\right) \otimes \sigma_{3},
$$

where $H_{\mathrm{SSH}}(k, t)=(t+\cos k) \sigma_{1}+\sin k \sigma_{2}$ is the $\mathrm{SSH}$ Hamiltonian. This model respects the reflection symmetries $\hat{m}_{x}=\sigma_{1} \otimes \sigma_{0}$ and $\hat{m}_{y}=\sigma_{3} \otimes \sigma_{1}$, the chiral symmetry $\Pi=\sigma_{3} \otimes \sigma_{3}$, the particle-hole symmetry $\Xi=\sigma_{3} \otimes \sigma_{3} \kappa$ and the time-reversal symmetry $\Theta=\kappa$. At the high-symmetric momenta for $k_{x}$ (similarly for $k_{y}$ ), the Hamiltonian can be written in a basis $\beta$ consisting of eigenvectors of $\hat{m}_{x}$ as

$$
\left[H_{\mathrm{BBH}}\left(k_{x}=k^{*}, k_{y}\right)\right]_{\beta}=H_{1} \oplus H_{2} \text {. }
$$

Specifically, we choose $\beta=\left\{\left|\uparrow_{1} \uparrow_{3}\right\rangle,\left|\uparrow_{1} \downarrow_{3}\right\rangle,\left|\downarrow_{1} \uparrow_{3}\right\rangle,\left|\downarrow_{1} \downarrow_{3}\right\rangle\right\}$ as the basis, where $\left|\uparrow_{\lambda}\right\rangle$ and $\left|\downarrow_{\lambda}\right\rangle$ are eigenvectors of $\sigma_{\lambda}$ with $\lambda=1,2,3$. In this basis, $H_{1}=H_{\mathrm{SSH}}\left(k_{y}, t_{y}\right)+\left(t_{x}+\cos k^{*}\right) \sigma_{3}$ and $H_{2}=H_{\mathrm{SSH}}\left(k_{y}, t_{y}\right)-\left(t_{x}+\cos k^{*}\right) \sigma_{3}$. Here $H_{1}$ has zeroenergy modes localized at boundaries in a geometry with open boundaries if and only if $C_{1}=\pi$ for $H_{1}$. This occurs only when $t_{x}=-\cos k^{*}$ so that $H_{1}=H_{\mathrm{SSH}}$ that preserves the timereversal symmetry, the particle-hole symmetry and the chiral symmetry. In other words, the Wannier band $v_{y}\left(k_{x}\right)$ and the $y$-normal edge energy spectra close their gaps simultaneously at $k_{x}=\pi\left(k_{x}=0\right)$ when $t_{x}=1\left(t_{x}=-1\right)$ and $\left|t_{y}\right|<1$. In addition, if we add a term $c \sin k_{y} \sigma_{1} \otimes \sigma_{0}$ with $c$ being a real number, which preserves two reflection symmetries and the chiral symmetry, it results in a new term $c \sin k_{y} \sigma_{0}$ in $H_{1}$. This term still respects the particle-hole symmetry and the $P T=\sigma_{1} \kappa$ symmetry so that the correspondence between the Berry phase of $\pi$ and the existence of zero-energy edge modes persists $[68,69]$.

However, remarkably, we find that the correspondence does not necessarily hold when the next-nearest-neighbor intercell hopping is involved. For instance, consider the following model:

$$
\begin{aligned}
H_{\mathrm{NNN}}\left(k_{y}\right)= & \left(\gamma+\cos k_{y}\right) \sigma_{3}+\left(\sin k_{y}+b_{2} \sin 2 k_{y}\right) \sigma_{1} \\
& +b_{2} \cos 2 k_{y} \sigma_{2},
\end{aligned}
$$

where $\gamma$ and $b_{2}$ are real parameters with $b_{2}$ denoting the strength of the next-nearest-neighbor intercell hopping. When $b_{2}=0$, the Hamiltonian can be obtained by applying a unitary transformation to $H_{\mathrm{SSH}}$, and hence zero-energy edge modes arise if and only if the Berry phase is equal to $\pi$. However, when $b_{2} \neq 0$, we find that both of these two questions can be answered no. Figure 4 illustrates that when $C_{1}=\pi$, the energy spectra of the boundary states remain gapped, and when the edge energy gap vanishes, $C_{1} \neq \pi$. This suggests that the correspondence between the Wannier band and edge energy gaps may break down when the long-range hopping is involved in a QTI, leading to the emergence of the type-II QTI.

\section{B. Simplified models for the type-II QTI}

By enforcing the reflection symmetries $\hat{m}_{x}=\sigma_{1} \otimes \sigma_{3}$, $\hat{m}_{y}=\sigma_{1} \otimes \sigma_{1}$ and the particle-hole symmetry $\Xi=\sigma_{3} \otimes \sigma_{0} \kappa$, we can construct a quadrupole topological model based on 

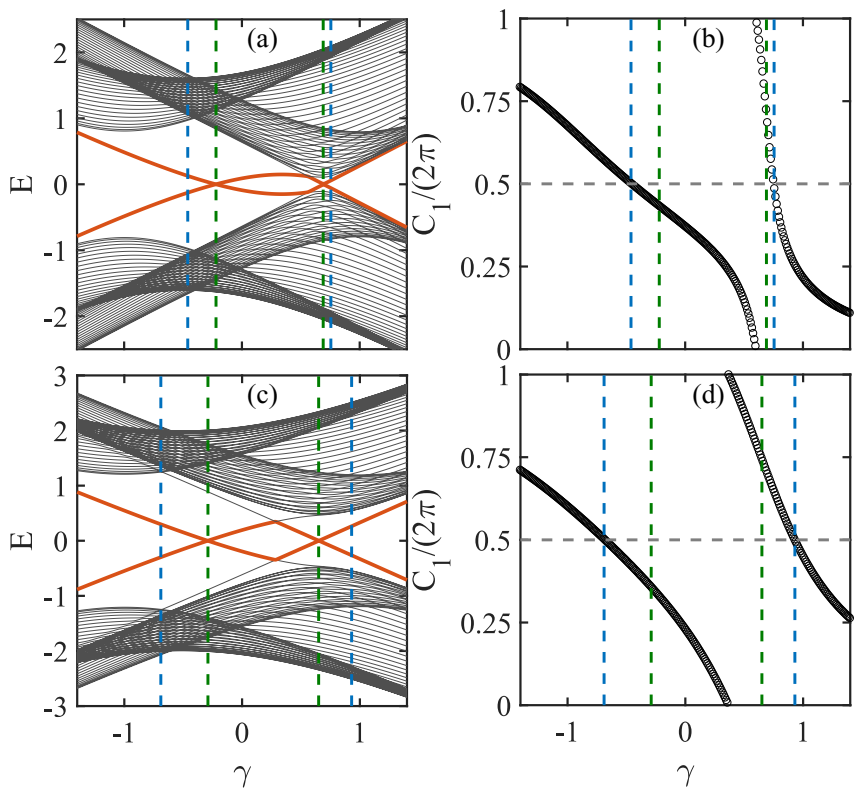

FIG. 4. [(a) and (c)] The energy spectra of the Hamiltonian $H_{\mathrm{NNN}}$. [(b) and (d)] The Berry phase of the occupied band for the corresponding Hamiltonian. In (a) and (b) $b_{2}=0.8$ and in (c) and (d) $b_{2}=1.2$. The green lines show the value of $\gamma$ where zero-energy edge modes arise, and the blue lines show the value of $\gamma$ where the Berry phase is equal to $\pi$. These lines do not coincide, implying that the correspondence between $C_{1}=\pi$ and the existence of zero-energy edge states breaks down.

$H_{\mathrm{NNN}}$ and a transformed SSH model as

$$
H_{I I}=H_{L}+\sigma_{2} \otimes H_{\mathrm{SSH}}^{\prime}\left(k_{x}\right)
$$

where

$$
\begin{aligned}
H_{L}= & \left(\gamma+\cos k_{y}\right) \sigma_{1} \otimes \sigma_{0}+\left(\sin k_{y}+b_{2} \sin 2 k_{y}\right) \sigma_{3} \otimes \sigma_{1} \\
& +b_{2} \cos 2 k_{y} \sigma_{3} \otimes \sigma_{2},
\end{aligned}
$$

and $H_{\mathrm{SSH}}^{\prime}\left(k_{x}\right)=\left(\gamma+t_{x}-g_{0}+t_{x} \cos k_{x}\right) \sigma_{2}+t_{x} \sin k_{x} \sigma_{3}$ can be obtained by applying a unitary transformation to $H_{\mathrm{SSH}}\left(k_{x}\right)$. Here we set $t_{x}=1$. The Hamiltonian (20) has exactly the same reflection symmetries and the particle-hole symmetry as the Hamiltonian (1). Yet, compared to the latter, this Hamiltonian is significantly simplified.

When $k_{x}=\pi$, the Hamiltonian can be written as

$$
\left[H_{I I}\right]_{\beta}=\left[\begin{array}{cc}
H_{\mathrm{NNN}}\left(k_{y}\right)+t_{0} \sigma_{1} & 0_{2 \times 2} \\
0_{2 \times 2} & \sigma_{3} H_{\mathrm{NNN}}\left(-k_{y}\right) \sigma_{3}-t_{0} \sigma_{1}
\end{array}\right]
$$

in a basis consisting of eigenvectors of $\hat{m}_{x}$, i.e., $\beta=$ $\left\{\left|\uparrow_{x} \uparrow_{z}\right\rangle,\left|\downarrow_{x} \downarrow_{z}\right\rangle,\left|\uparrow_{x} \downarrow_{z}\right\rangle,\left|\downarrow_{x} \uparrow_{z}\right\rangle\right\}$. Here $t_{0}=\gamma-g_{0}$. Adding the term $t_{0} \sigma_{1}$ into $H_{\mathrm{NNN}}\left(k_{y}\right)$ only shifts the critical value of $\gamma$ where zero-energy edge modes appear. For simplicity and clarity, we therefore set $g_{0}=\gamma_{c}$ so that $t_{0}=0$ when $\gamma=\gamma_{c}$ where there exist zero-energy edge modes for $H_{\mathrm{NNN}}$ under open boundary conditions. For example, when $b_{2}=0.8\left(b_{2}=\right.$ $1.2)$, we set $g_{0}=-0.22\left(g_{0}=-0.29\right)$ as shown in Fig. 4. Now we can clearly see that for this model while the $y$-normal edge energy gap vanishes when $\gamma=\gamma_{c}$, the Wannier band gap does not close at $v_{y}= \pm 1 / 2$.
To see that the type-II QTI indeed emerges in this simplified model, we numerically compute the quadrupole moment, the energy spectra under open boundary conditions along all directions and the edge polarizations, displaying them in Fig. 5. Evidently, when $\gamma$ decreases across $\gamma_{c}=-0.22$, the zero-energy corner modes appear due to the $y$-normal edge energy gap closing, showing that the system enters into a higher-order topological insulating phase. Meanwhile, the quadrupole moment suddenly jumps to $0.5 e$ at $\gamma_{n c}=-0.26$ for a $N \times N$ system with $N=240$ denoting the number of unit cells along $x$ (or $y$ ). The discrepancy from the edge energy gap closing point $\gamma_{c}=-0.22$ is attributed to the finite-size effects while calculating the quadrupole moment in real space. Specifically, in Fig. 5(e), we display the finite-size scaling between $\left|\gamma_{n c}-\gamma_{c}\right|$ and the system size $N$, where $\gamma_{c}$ and $\gamma_{n c}$ denote the point where the edge energy gap closes and the phase transition point determined by the quadrupole moment for a finite system, respectively. The relation exhibits a power law decaying, i.e., $\left|\gamma_{n c}-\gamma_{c}\right| \propto N^{-\delta}$ with $\delta=0.636$, implying that $\gamma_{n c}=\gamma_{c}$ in the thermodynamic limit. This tells us that the higher-order topological insulator is a quadrupole topological insulating phase. Such an edge energy gap closing also leads to the emergence of the edge polarizations $p_{x}^{\text {edge }}$ at the boundaries vertical to $y$ [see the inset in Fig. 5(a) and Fig. 5(d)]. But the edge polarizations $p_{y}^{\text {edge }}$ at the $x$-normal boundaries remain zero because the Wannier bands $v_{y}$ remain gapped at $v_{y}=$ $\pm 1 / 2$. As a consequence, the type-II quadrupole insulating phase arises. Compared to the type-II phase introduced in Sec. II, it is normal in the sense that the Wannier spectra $v_{x}$ have edge states only at $v_{x}= \pm 1 / 2$. We also calculate the edge polarizations based on the formula (15) by choosing a gauge such that $W_{v_{x}}^{\epsilon=\pi}\left(\gamma_{0}=-0.8\right)=W_{v_{y}}^{\epsilon=\pi}\left(\gamma_{0}=-0.8\right)=0$, showing that the winding number can correctly characterize the edge polarization change due to the Wannier band closing or the bulk energy gap closing. For the edge polarization $p_{x}^{\text {edge }}$, it changes at $\gamma_{c}=-0.22$ because of the edge energy gap closing associated with a one time change of a parity (eigenvalue of $\hat{m}_{x}$ ) at a $y$-normal boundary and the quadrupole moment.

Similarly, we can construct another model

$$
H_{I I I}=H_{L}-\sigma_{3} \otimes H_{\mathrm{SSH}}^{\prime}\left(k_{x}\right),
$$

which respects the reflection symmetries $\hat{m}_{x}$ and $\hat{m}_{y}$, the chiral symmetry $\Pi_{2}$, i.e., $\Pi_{2} H_{I I I} \Pi_{2}^{-1}=-H_{I I I}$ with $\Pi_{2}=\sigma_{2} \otimes \sigma_{0}$ and the particle-hole symmetry $\Xi$. We also find the type-II quadrupole topological insulating phase in this model (see the Appendix E for the phase diagram). If we add a term $\sin k_{x} \sigma_{1} \otimes \sigma_{1}$ which breaks the particle-hole symmetry but preserves the chiral symmetry, then the type-II QTI also arises (see the Appendix E for the phase diagram). The above results demonstrate that the type-II QTI generically exists in systems with reflection symmetries as well as the particle-hole or chiral symmetry in the presence of long-range hopping.

\section{A new topological phase with nonzero quantized edge polarizations but without zero-energy corner modes}

We have demonstrated that the Wannier gap closing can drive the topological phase transition from the type-II QTI 

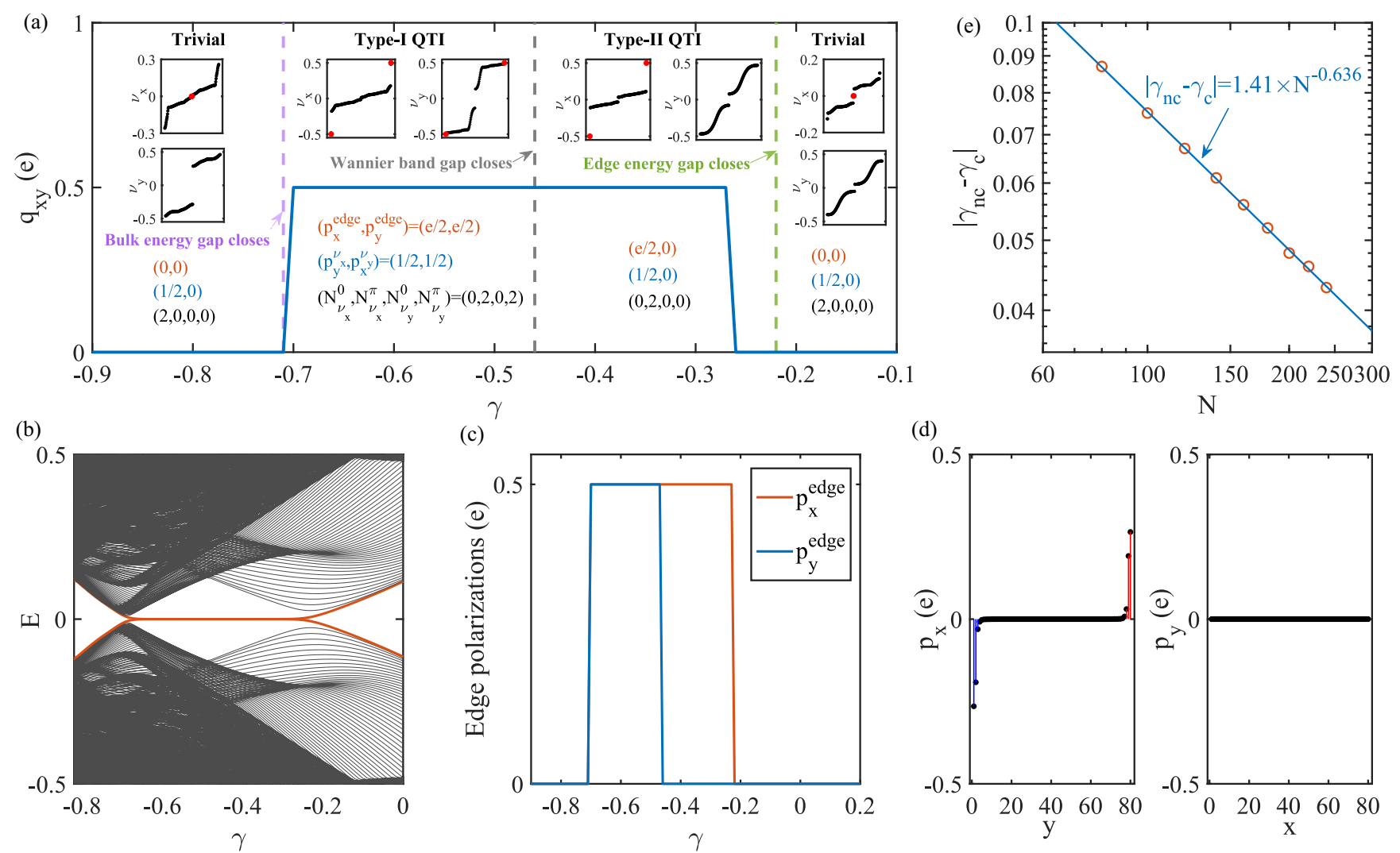

FIG. 5. (a) Phase diagram for the Hamiltonian $H_{I I}$ with respect to a system parameter $\gamma$, where the quadrupole moment is plotted as a blue line. In the phase diagram, we observe the topologically trivial insulator, the type-I QTI and the type-II QTI. The subsets display the same quantities as in Fig. 2. (b) The energy spectrum as a function of $\gamma$ for open boundary conditions along all directions with zero-energy corner modes being highlighted by a red line. (c) The edge polarizations calculated based on the formula (15) by choosing a gauge such that $W_{v_{x}}^{\epsilon=\pi}\left(\gamma_{0}=-0.9\right)=W_{v_{v}}^{\epsilon=\pi}\left(\gamma_{0}=-0.9\right)=0$ given that the phase is topologically trivial when $\gamma=-0.9$. (d) The spatial profiles of the edge polarizations in a type-II QTI with $\gamma=-0.35$. (e) $\left|\gamma_{n c}-\gamma_{c}\right|$ with respect to the system size $N$ in the logarithmic scale, showing $\left|\gamma_{n c}-\gamma_{c}\right| \propto$ $N^{-\delta}$ with $\delta=0.636 . \gamma_{c}$ and $\gamma_{n c}$ denote the point where the edge energy gap closes and the phase transition point determined by the quandruple moment for a finite system, respectively. Here $b_{2}=0.8$. See Appendix E for the phase diagram for $b_{2}=1.2$.

to the type-I and vice versa, suggesting that the topological property change of the Wannier band can lead to new topological phases. In contrast to the QTI, one may wonder whether the Wannier band gap closing can result in a new topological phase with nonzero quantized edge polarizations but without quadrupole moments and zero-energy corner modes. In the following, we will show the existence of this phase by considering the Hamiltonian in momentum space

$$
\begin{aligned}
H_{I V}= & \left(\gamma+\cos k_{y}\right) \sigma_{1} \otimes \sigma_{0}+\left(\sin k_{y}+b_{2} \sin 2 k_{y}\right) \sigma_{2} \otimes \sigma_{1} \\
& +\sigma_{2} \otimes\left[\left(1+b_{2} \cos 2 k_{y}+t_{x} \cos k_{x}\right) \sigma_{2}+t_{x} \sin k_{x} \sigma_{3}\right],
\end{aligned}
$$

which respects the reflection symmetries $\hat{m}_{x}$ and $\hat{m}_{y}$, the timereversal symmetry $\Theta$, the particle-hole symmetry $\Xi$ and the chiral symmetry $\Pi$. Here we set $t_{x}=1$.

Figure 6 illustrates that the Wannier spectra $v_{y}$ close their gap at $v_{y}= \pm 1 / 2$ when $\gamma=-0.45$ and the Wannier spectra $v_{x}$ remain gapped. This gap closure results in edge states in the Wannier bands at $v_{y}= \pm 1 / 2$ under open boundary conditions, giving rise to the quantized edge polarization $p_{y}^{\text {edge }}= \pm e / 2$ [their spatial distributions are shown in the inset of Fig. 6(c)]. However, the energy spectra of the system with open bound- aries in all directions remain gapped until $\gamma=-0.22$. This means that a new topological phase arises when $-0.45<\gamma<$ -0.22 . In this phase, despite the vanishing of the quadrupole moment and the absence of the zero-energy corner modes, nonzero quantized edge polarizations exist. As $\gamma$ increases across -0.22 , the quadrupole moment and the corner modes appear, leading to the type-I QTI. In addition, we display the edge polarizations in Fig. 6(c) evaluated based on the formula (15) by choosing a gauge such that $W_{v_{x}}^{\epsilon=\pi}\left(\gamma_{0}=-0.8\right)=$ $W_{v_{y}}^{\epsilon=\pi}\left(\gamma_{0}=-0.8\right)=0$, implying that the winding number correctly characterizes the change of the edge polarizations across the topological phase transition at $\gamma=-0.45$.

Interestingly, when we include a term $\sin k_{x} \sigma_{1} \otimes \sigma_{1}$ that breaks the time-reversal symmetry and the particle-hole symmetry but preserves the chiral symmetry, or a term $\sin k_{x} \sigma_{3} \otimes$ $\sigma_{3}$ that breaks the time-reversal symmetry and the chiral symmetry but preserves the particle-hole symmetry, we can still observe the new topological phase transition (see Appendix E for details).

The discovery of the type-II QTI and the new topological phase discussed above suggests that the gap closing of the Wannier spectra can induce topological phase transitions without involving any energy gap closing. 

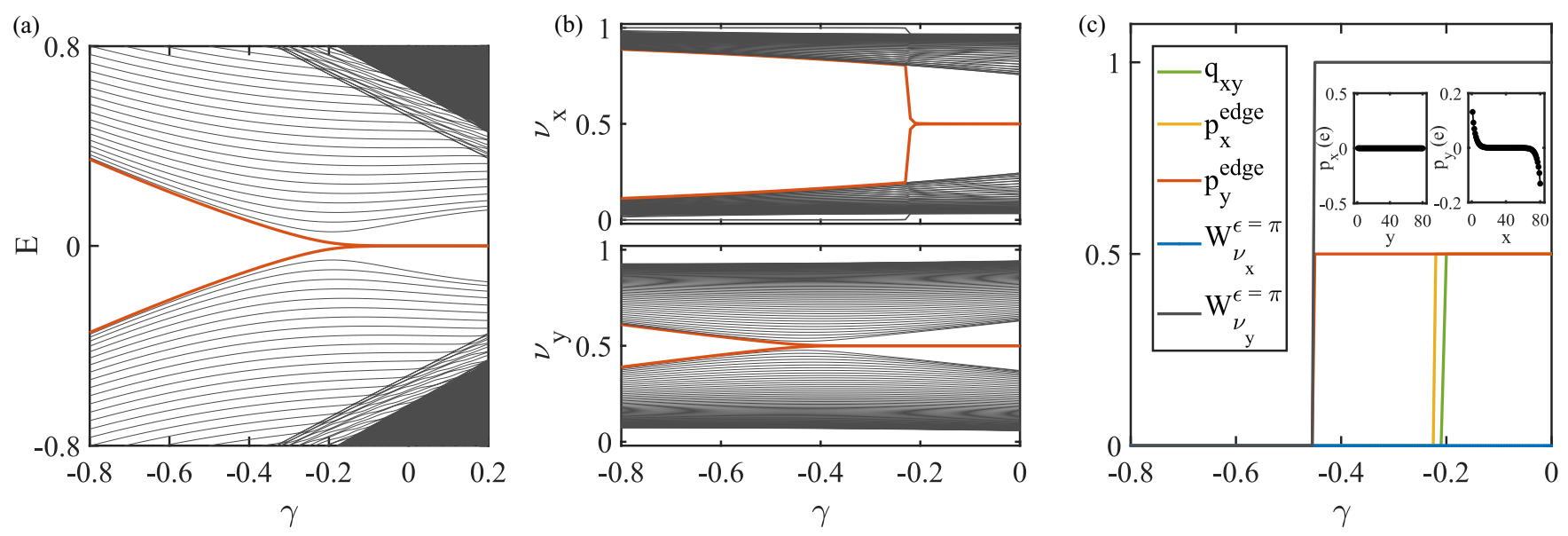

FIG. 6. (a) The energy spectrum versus $\gamma$ for the Hamiltonian $H_{I V}$ with open boundaries in all directions. (b) The Wannier band $v_{x}$ and $v_{y}$ versus $\gamma$ under open boundary conditions along $y$ and $x$, respectively. The red lines depict the edge states in the Wannier spectrum. (c) The quadrupole moment $q_{x y}$, the edge polarizations $p_{\mu}^{\text {edge }}(\mu=x, y)$ computed using the formula (15) and the winding numbers $W_{v_{\mu}}^{\epsilon=\pi}(\mu=x, y)$ computed using the formula (13) versus $\gamma$. When calculating the edge polarizations and the winding number, we choose a gauge such that $W_{v_{x}}^{\epsilon=\pi}\left(\gamma_{0}=-0.8\right)=W_{v_{y}}^{\epsilon=\pi}\left(\gamma_{0}=-0.8\right)=0$ because the phase is topologically trivial when $\gamma=-0.8$. The figure demonstrates the existence of a new topological phase with nonzero $p_{y}^{\text {edge }}$ but without quadrupole moments and zero-energy corner modes when $-0.45<\gamma<-0.22$

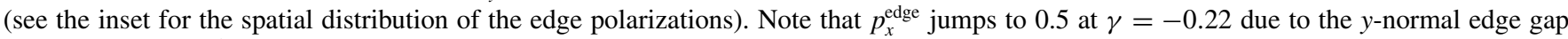
closing. $q_{x y}$ and $p_{x}^{\text {edge }}$ are hidden behind the blue line when $\gamma<-0.22$. The small discrepancy between the quadrupole moment transition point ( $N=80$ for evaluation of the quadrupole moment) and the edge gap closing point is due to the finite-size effects. Here $b_{2}=1.2$.

\section{An alternative approach to show the inequivalence}

Alternatively, we may consider the problem based on the method introduced in Ref. [28]. There, it has been shown that the edge spectrum can be continuously deformed into the Wannier band, implying that they share the same spectral flow, e.g., a chiral edge mode in a quantum Hall insulator corresponds to a winding Wannier band. Based on the method, Ref. [27] generalizes the correspondence between the Wannier and edge spectra in the quadrupole topological insulating phase by showing that the Wannier gap and boundary spectra gap have to close simultaneously. This also indicates that the type-II QTI cannot occur. However, Ref. [27] only studies a specific model with the nearest-neighbor intercell hopping.

In the following, we will show that these two gaps do not necessarily vanish at the same time in our model Hamiltonian (1) by following the method in Refs. [27,28]. Let us consider a gapped Hamiltonian $H(\mathbf{k})$ in momentum space. We define the projection operator to the occupied bands as $\tilde{P}(\mathbf{k})=\sum_{n=1}^{N_{\text {occ }}}\left|u_{\mathbf{k}}^{n}\right\rangle\left\langle u_{\mathbf{k}}^{n}\right|$ with $\left|u_{\mathbf{k}}^{n}\right\rangle$ being an occupied eigenstate of $H(\mathbf{k})$. The representation of this operator in the real space is obtained by Fourier transformation in the direction perpendicular to an edge,

$$
P_{r \alpha, s \beta}\left(\mathbf{k}_{\|}\right)=\int_{B Z} \frac{d k_{\perp}}{2 \pi} e^{i k_{\perp}(r-s)} \tilde{P}_{\alpha, \beta}(\mathbf{k}),
$$

where $r, s$ are coordinates of lattices in the edge-normal direction and $\alpha, \beta$ are orbital indices. $\mathbf{k}_{\|}$and $k_{\perp}$ denote the momentum parallel and perpendicular to the edge, respectively.

Following Refs. [27,28], we construct a Hamiltonian from the projection operator,

$$
H_{e}^{0}=P V_{0}(x) P+(I-P),
$$

where

$$
V_{0}(x)=\left\{\begin{array}{rl}
1 & \text { for } x<0 \\
-1 & \text { for } x \geqslant 0
\end{array} .\right.
$$

For a tight-binding model, $x$ is the coordinate of discrete lattice sites along the direction perpendicular to the edge and takes the value of integer numbers from $-N_{x} / 2$ to $\left(N_{x} / 2-1\right)$ with $N_{x}$ being the size along the $x$ direction. Here $V_{0}(x)$ introduces two boundaries at $x=0$ and $x=N_{x} / 2$ so that for $-N_{x} / 2 \leqslant x<0$, the system is topologically trivial and, otherwise, topologically equivalent to $H(\mathbf{k})$. Indeed, we have found that the eigenvalues $E_{V_{0}}\left(k_{y}\right)$ of $H_{e}^{0}\left(k_{y}\right)$ can be adiabatically mapped to the energy spectrum of the original Hamiltonian under open boundary conditions along $x$.

To see the connection between the edge energy spectrum and the Wannier band, we impose a linear edge so that the Hamiltonian reads

$$
H_{e}^{L}=P V_{L}(x) P+M(I-P),
$$

where the deformed linear edge potential (see Fig. 7) is given by

$$
V_{L}(x)= \begin{cases}x+N_{x} / 2 & \text { for }-N_{x} / 2 \leqslant x<-N_{x} / 2+M \\ M & \text { for }-N_{x} / 2+M \leqslant x<-M+1 \\ -x & \text { for }-M+1 \leqslant x<M \\ -M & \text { for } M \leqslant x<N_{x} / 2-M+1 \\ x-N_{x} / 2 & \text { for } N_{x} / 2-M+1 \leqslant x<N_{x} / 2\end{cases}
$$

Here $M$ is used to control the size of the linear potential region. When $M=1, V_{L}(x)$ is only slightly deformed from $V_{0}(x)$. Reference [28] argues that this slight change should 

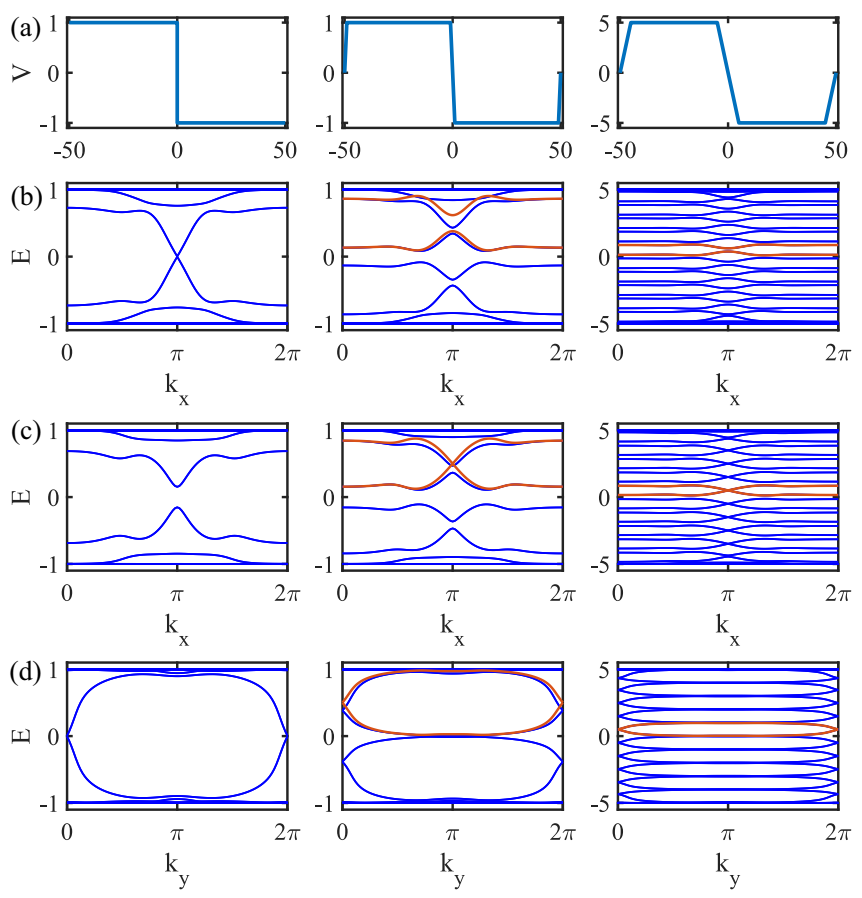

FIG. 7. The connection between the edge energy spectrum and Wannier spectrum. (a) Visualization of the edge potential for $V_{0}$ in the left column as well as $V_{L}$ with $M=1$ and $M=5$ in the middle and right columns, respectively. (b) The energy bands of $H_{e}^{0}\left(H_{e}^{L}\right)$ with $V_{0}(y)\left[V_{L}(y)\right]$ versus $k_{x}$ in the left column (in the middle and right columns) for $\gamma=0.34$. The edge energy spectrum encoded in $H_{e}^{0}$ has a vanishing gap, while the Wannier spectrum encoded in $H_{e}^{L}$ for a moderate or large $M$ (e.g., $M=5$ ) has a finite gap. (c) The same spectrum as in (b) for $\gamma=0.12$. The edge energy spectrum has a finite gap, which gradually vanishes as $M$ is increased for $V_{L}(y)$, leading to a gapless Wannier spectrum. (d) The energy spectrum of $H_{e}^{0}\left(H_{e}^{L}\right)$ with $V_{0}(x)\left(V_{L}(x)\right)$ versus $k_{y}$ in the left column (in the middle and right columns). In this scenario, both the edge energy spectrum and Wannier spectrum are gapless. In the middle and right columns for (b)-(d), the Wannier spectra are plotted as the red lines in comparison with the energy spectrum of $H_{e}^{L}$, showing that the former agrees very well with the latter for $M=5$. For (b)-(d), the middle and right columns correspond to the results for $M=1$ and $M=5$, respectively.

not significantly change the energy spectrum and thus as one continuously deform the boundary by increasing $M$ for $V_{L}(x)$, the energy spectrum should be smoothly deformed into the eigenvalues of $P x P$, the restriction of which to the interval $[0,1]$ gives exactly the Wannier spectrum. Reference [27] generalizes this argument to the higher-order topological case by assuming that this slight change should not open an energy gap if the boundary states using $V_{0}(x)$ are gapless, and concludes that the edge energy spectrum and the Wannier spectrum should close their gaps simultaneously.

However, we find that this generalized argument is not always true. For example, in our model, when $\gamma=0.34$, the energy spectrum is gapless under the potential $V_{0}(y)$, which is consistent with our results under open boundary condition along $y$. But, as we slightly deform the boundaries, e.g., taking $M=1$ for $V_{L}(y)$, we find that the energy gap opens, as shown in Fig. 7(b). When we further increase $M$ to 5, the gap remains open and the energy spectrum is exactly the same as the Wannier spectrum.

We also show that for $\gamma=0.12$, the energy gap vanishes for $M=5$ while there is a nonzero energy gap for the energy spectrum under the boundary geometry $V_{0}(y)$ [see Fig. 7(c)]. We indeed also find the case where the energy gap always remains gapless. For example, for $\gamma=1.03$, the gap remains zero as we continuously deform the boundaries, as shown in Fig. 7(d).

Therefore, we conclude that the edge energy bands and Wannier bands are not guaranteed to close their gaps simultaneously and thus can be topologically inequivalent.

\section{PUMPING PHENOMENA AND NOVEL THREE-DIMENSIONAL HIGHER-ORDER TOPOLOGICAL INSULATORS}

We now discuss the pumping phenomena as a system parameter is slowly tuned. We expect the existence of anisotropic edge currents during a pumping process. To induce the change of the edge polarization, we need to break the reflection symmetry by adding a $\delta \tau_{z} \sigma_{0}$ term so that the edge polarization is not locked to be quantized. However, to maintain the vanishing of the bulk polarization, we still preserve the inversion symmetry. We also maintain the bulk and edge energy gaps during the entire cycle for adiabaticity.

Specifically, we consider the pumping process across the topologically trivial phase and type-II AQTI in Fig. 2. To achieve the pumping, we choose $\delta=0.1 \sin (t)$ and $\gamma=$ $0.35+0.1 \cos (t)$ in the Hamiltonian (1). At $t=0,2 \pi$, the system is in the topologically trivial phase without any edge polarization, quadrupole moments and corner charges, while at $t=\pi$, it is in the type-II AQTI with $q_{x y}=\left|Q^{\text {corner }}\right|=$ $\left|p_{x}^{\text {edge }}\right|=1 / 2$ and $p_{y}^{\text {edge }}=0$. As time progresses with changes of $\delta$ and $\gamma$, the system evolves from the topologically trivial phase to the type-II quadrupole insulating phase and then return to the original trivial phase. At each time, we evaluate the edge polarization, corner charge and quadrupole moment. We find that during an entire cycle, the edge polarization at the top boundary increases by one and at the bottom boundary it decreases by one, as shown in Fig. 8(a). This also happens for corner charges and the quadrupole moment. However, the left and right edges do not exhibit a net transport for the polarization.

We also consider the pumping process across the type-I AQTI and type-II AQTI described by $\delta=0.1 \sin (t)$ and $\gamma=$ $0.1+0.1 \cos (t)$. At $t=0,2 \pi$, the system is in the type-II AQTI phase while at $t=\pi$, the system in the type-I AQTI phase. As time evolves over a full cycle, it turns out that only the left and right boundaries show a net change of the polarization but not for the other quantities such as the edge polarization at the top and bottom boundaries, corner charges and quadrupole moments, as shown in Fig. 8(b). Both of the pumping phenomena contrast with previous works where all boundaries, corner charges and quadrupole moments exhibit a net change during a cycle $[1,2,61,62]$. These pumping phenomena indicates the peculiar features of the type-II AQTI.

If we regard the adiabatic parameter $t$ as momentum $k_{z}$ in the third direction, then we obtain two three-dimensional 

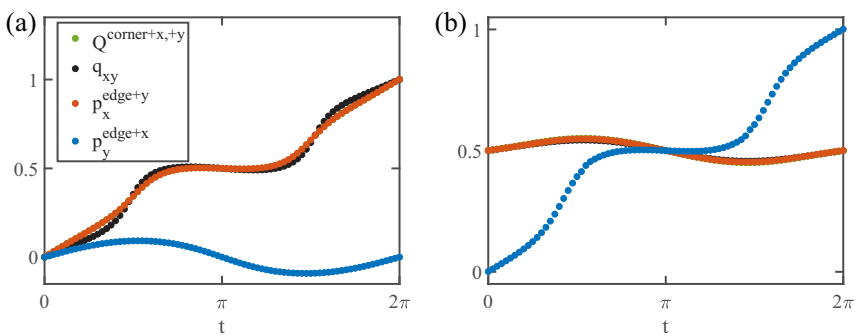

FIG. 8. The transport of the quadrupole moment $q_{x y}$, corner charge $Q^{\text {corner }+\mathrm{x},+\mathrm{y}}$ and edge polarization $p_{x, y}^{\text {edge } \alpha}$ over a full cycle. (a) The cycle refers to the evolution of a system from a topologically trivial phase to the type-II AQTI and finally return. Note that the green line is hidden behind the red one. (b) The cycle refers to the evolution of a system from the type-II phase to the type-I AQTI and then return. Note that the green and black lines are hidden behind the red one. The units of all the quantities are $e$.

higher-order topological insulators characterized by a set of topological invariants consisting of the winding of the quadrupole moment and edge polarizations along two directions: $\left(N_{q_{x y}}, N_{p_{x} \text { edge }}, N_{p_{y}^{\text {edge }}}\right)$, where $N_{O}=\int_{0}^{2 \pi} d k_{z} \frac{\partial O}{\partial k_{z}}$ with $O=q_{x y}, p_{x, y}^{\text {edge }}$. The new insulating phases correspond to $\left(N_{q_{x y}}, N_{p_{x} \text { edge }}, N_{p_{y} \text { edge }}\right)=(1,1,0)$ and $(0,0,1)$, respectively, which are fundamentally different from the previously found insulator with $(1,1,1)$. Although the phase with the winding number being $(0,0,1)$ has a winding for the edge polarization, it does not lead to the chiral hinge modes beyond the conventional wisdom that the presence of the winding of the polarization corresponds to a Chern insulator with chiral edge modes. In fact, in our case, it is associated with the presence of chiral edge modes in the Wannier bands, as presented in Appendix F.

\section{QUENCH DYNAMICS}

Since these new topological phase transitions are driven by the Wannier gap closure, they may arise from quench dynamics through unitary time evolution. In this section, we will study the dynamics of states as the Hamiltonian is suddenly changed from one phase to another. Specifically, we consider the type-I quadrupole Hamiltonian [1,2]

$$
\begin{aligned}
H_{\mathrm{BBH} 0}(\mathbf{k})= & \left(\gamma_{x}+\lambda \cos k_{x}\right) \Gamma_{4}+\lambda \sin k_{x} \Gamma_{3} \\
& +\left(\gamma_{y}+\lambda \cos k_{y}\right) \Gamma_{2}+\lambda \sin k_{y} \Gamma_{1},
\end{aligned}
$$

where $\Gamma_{j}=-\tau_{2} \sigma_{j}(j=1,2,3)$ and $\Gamma_{4}=\tau_{1} \sigma_{0}$. This Hamiltonian respects the reflection symmetries $\hat{m}_{x}$ and $\hat{m}_{y}$, the timereversal symmetry $\Theta$, the particle-hole symmetry $\Xi$ and the chiral symmetry $\Pi$. The phase diagram is shown in Fig. 9 with respect to $\gamma_{x} / \lambda$ and $\gamma_{y} / \lambda$ (see also Ref. [2]). We choose the ground state $\left|\psi_{i}\right\rangle$ of $H_{i} / \gamma_{x}=\tau_{1} \sigma_{0}-\tau_{2} \sigma_{2}$ (i.e., $\gamma_{x}=\gamma_{y}$ and $\lambda=0)$ as the initial state and then suddenly tune $\gamma_{x}, \gamma_{y}$ and $\lambda$ to the values as shown in Fig. 9(a) so that the Hamiltonian changes to $H_{f}(\mathbf{k})$. The state then evolves under the final Hamiltonian, i.e., $\left|\psi_{\mathbf{k}}(t)\right\rangle=e^{-i H_{f}(\mathbf{k}) t}\left|\psi_{i}\right\rangle$. Since the evolving state $\left|\psi_{\mathbf{k}}(t)\right\rangle$ is an eigenstate of a parent Hamiltonian defined as $H_{p}(\mathbf{k})=e^{-i H_{f}(\mathbf{k}) t} H_{i}(\mathbf{k}) e^{i H_{f}(\mathbf{k}) t}$, the topological properties of the evolving states are determined by the parent Hamiltonian.
During time evolution, one can easily find that the parent Hamiltonian still preserves the reflection symmetries, i.e., $\hat{m}_{\mu} H_{p} \hat{m}_{\mu}^{\dagger}=H_{p}\left(k_{\mu} \rightarrow-k_{\mu}\right)$ with $\mu=x, y$, and the particlehole symmetry, i.e., $\Xi H_{p} \Xi^{-1}=-H_{p}(-\mathbf{k})$, but usually breaks the time-reversal symmetry and the chiral symmetry. Without loss of generality, we study two scenarios: one corresponds to the final Hamiltonian in the topologically trivial region and the other in the quadrupole insulating region.

Figures 9(b1)-9(d1) illustrate the entanglement spectrum, Wannier spectrum and edge polarizations as a function of time, after the Hamiltonian is quenched into a topologically trivial phase. At $t=1.1$, the gap of the entanglement spectrum $\mathrm{ES}_{x}$ vanishes, revealing the vanishing of the energy gap for the parent Hamiltonian under open boundary conditions along $x$ [70]. This gap closure leads to the appearance of the quantized quadrupole moment $\left(q_{x y}=e / 2\right)$ and edge polarizations along $y\left(p_{y}^{\text {edge }}= \pm e / 2\right)$. Here, the edge polarizations are calculated by the formula (15), where $\Delta N_{q \mu}(\mu=x, y)$ are evaluated by the number of times that the gap of $\operatorname{ES}_{\bar{\mu}}(\bar{\mu}=y, x$ if $\mu=x, y)$ closes. Here we choose a gauge such that $W_{v_{x}}^{\epsilon=\pi}(t=$ $0)=W_{v_{y}}^{\epsilon=\pi}(t=0)=0$ for calculation in the sense that the initial states are topologically trivial. Since there is neither gap closure for $\mathrm{ES}_{y}$ nor Wannier gap closure for $v_{x}$, p pxe remains zero. In addition, the nested entanglement spectra $\mathrm{ES}_{x y}$ exhibit zero-energy modes (see the blue region) (the nested entanglement spectrum $\mathrm{ES}_{x y}=0.5$ corresponds to the entanglement zero mode, see Appendix G), reflecting the existence of fractional corner charges $\left(Q^{\text {corner }}= \pm e / 2\right)$ for the parent Hamiltonian in a geometry with open boundary conditions. This shows that the system enters into a typeII quadrupole topological insulating region with the basic relation $\left(p_{y}^{\text {edge }}+p_{x}^{\text {edge }}-q_{x y}-Q^{\text {corner }}\right) \bmod (1)=0$ being violated. At $t=2.5, \mathrm{ES}_{x}$ experiences a gap closure, leading to a topologically trivial phase with zero quadrupole moment $\left(q_{x y}=0\right)$ and edge polarizations $\left(p_{y}^{\text {edge }}=p_{x}^{\text {edge }}=0\right)$.

Remarkably, shortly afterwards, the gap of the Wannier bands $v_{x}$ vanishes at $t=2.55$ and $v_{x}=0.5$, resulting in nonzero quantized edge polarizations $p_{x}^{\text {edge }}=e / 2$. However, in this phase, the entanglement spectra do not exhibit any gap closure, reflecting the absence of the edge energy gap closure for the parent Hamiltonian under open boundary conditions. This accounts for the absence of the quadrupole moment and zero-energy modes in the nested entanglement spectrum. When the gap of these Wannier bands $v_{x}$ closes again at $t=3.54$, the edge polarization $p_{x}^{\text {edge }}$ vanishes so that the topological phase becomes trivial. The appearance and disappearance of this new topological phase are caused only by the gap closure of the Wannier bands at $v=0.5$ (in other words, topological properties of the Wannier bands change due to the Wannier gap closure). This shows that the quench dynamics can produce new topological phases, although the coherent dynamics do not involve any energy gap closure.

Figures 9(b2)-9(d2) present the results for the final Hamiltonian in the type-I quadrupole insulating phase. We find that the states evolve into the type-II AQTI phase at $t=1.04$ with quantized quadrupole moments $\left(q_{x y}=e / 2\right)$ and quantized edge polarizations $p_{x}^{\text {edge }}= \pm e / 2$ but $p_{y}^{\text {edge }}=0$ due to closure of the gap of $\mathrm{ES}_{y}$. The nested entanglement spectrum has zero-energy modes in the region, reflecting the existence 
(a)

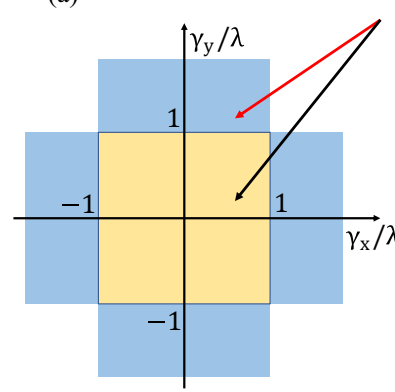

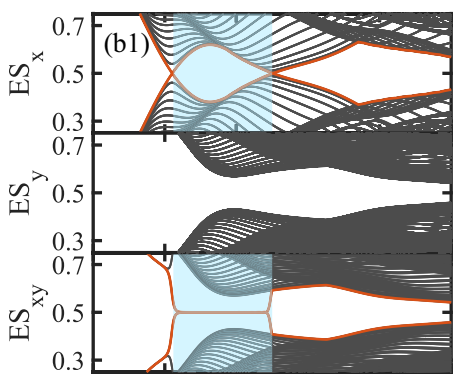
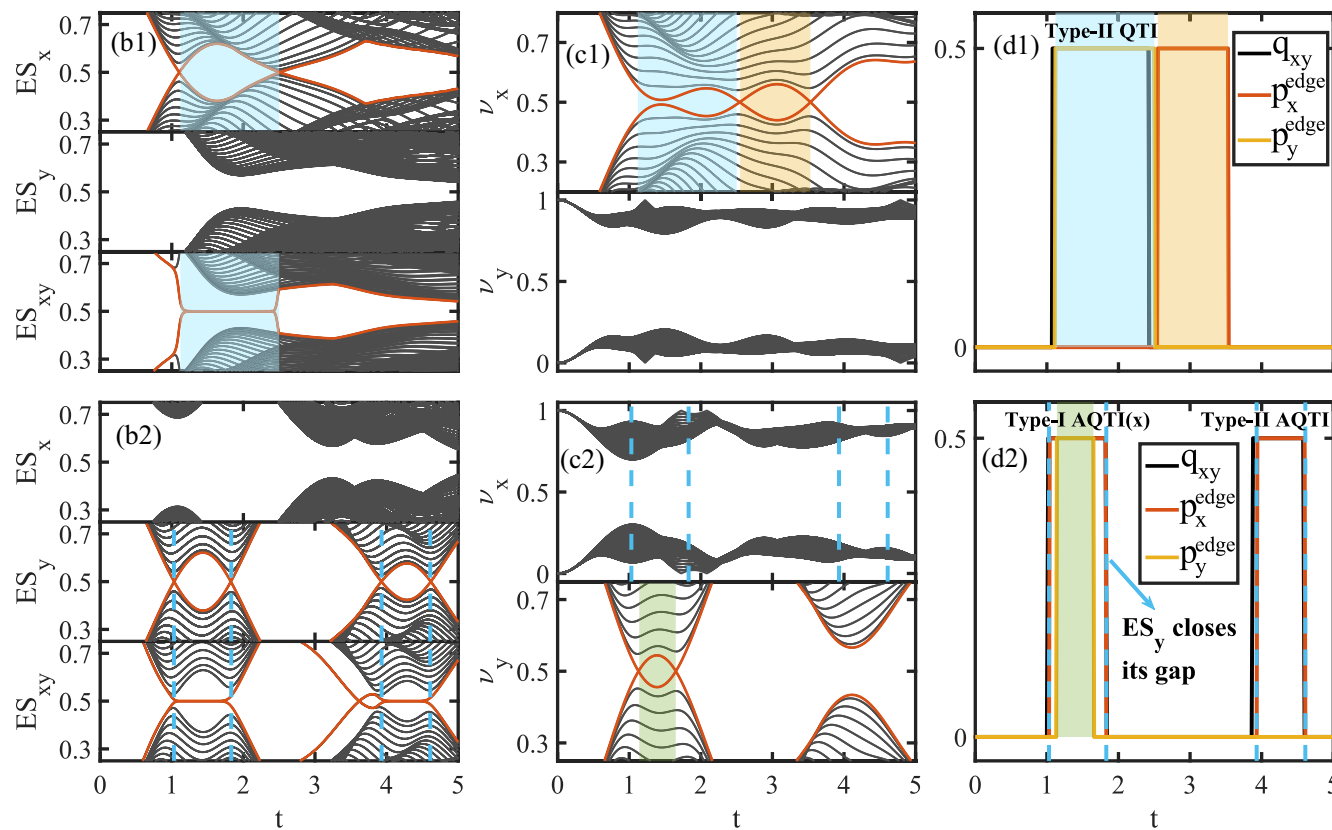
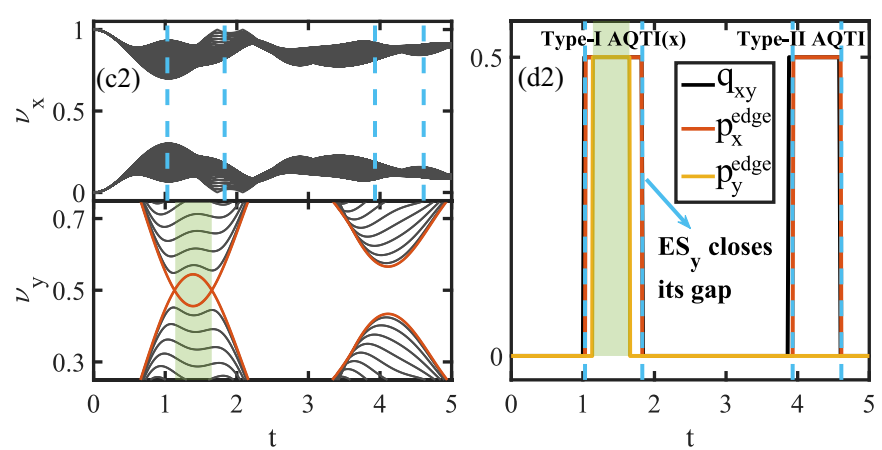

FIG. 9. (a) Phase diagram of the type-I Hamiltonian, where the yellow region corresponds to the type-I QTI and other regions to topologically trivial insulating phases. Note that while the Berry phase of the Wannier bands along one direction in the blue regions is nonzero, there are no edge polarizations since the Wannier bands close their gaps at either $v_{x}=0$ or $v_{y}=0$. The quench dynamics are performed by suddenly tuning system parameters from $\gamma_{x}=\gamma_{y}, \lambda=0$ to $\gamma_{x}=0.6, \gamma_{y}=1.2, \lambda=1$ and to $\gamma_{x}=0.6, \gamma_{y}=0.2, \lambda=1$, respectively (see the red and black arrows). (b1) Entanglement spectra, (c1) Wannier spectra, and (d1) quadrupole moments and edge polarizations as time evolves, for the quench dynamics denoted by the red arrow. [(b2)-(d2)] display the same physical quantities as (b1)-(d1), but for the quench dynamics denoted by the black arrow. In (b1) and (b2), $\mathrm{ES}_{x}\left(\mathrm{ES}_{y}\right)$ refers to the entanglement spectrum in the left (bottom) subsystem and $\mathrm{ES}_{x y}$ the nested entanglement spectrum (see Appendix G). The edge polarizations in (d1) and (d2) are calculated based on the formula (15) by choosing a gauge such that $W_{v_{x}}^{\epsilon=\pi}(t=0)=W_{v_{v}}^{\epsilon=\pi}(t=0)=0$ because the initial states are topologically trivial. In (b1)-(d1), the light blue region displays the type-II QTI (specifically, in this region, when $t<1.22$, it corresponds to the type-II AQTI, and when $t>1.22$, it corresponds to the type-II normal QTI) and the light red region displays the new topological phase with only quantized $p_{x}^{\text {edge }}$. In (b2)-(d2), the light green region shows the type-I AQTI(x) and the dashed blue lines label the time at which the gap of $\mathrm{ES}_{y}$ vanishes. The region between the two dashed blue lines around $t=4$ corresponds to the type-II AQTI phase. In (d1), $q_{x y}=0$ when $t<1.1$ or $t>2.43$, and in (d2), $q_{x y}=0$ when $t<1.04$ or $1.84<t<3.94$ or $t>4$.6. In (d1), the slight discrepancy between $q_{x y}$ and $p_{y}^{\text {edge }}$ around $t=2.5$ is caused by finite-size effects while calculating $q_{x y}$ in real space.

of fractional corner charges $\left(Q^{\text {corner }}= \pm e / 2\right)$ for the parent Hamiltonian under open boundary conditions. At $t=1.14$, the gap of the Wannier bands $v_{y}$ vanishes, yielding quantized edge polarizations $p_{y}^{\text {edge }}= \pm e / 2$, which signals the transition into the type-I AQTI where the basic relation $\left(p_{y}^{\text {edge }}+p_{x}^{\text {edge }}-\right.$ $\left.q_{x y}-Q^{\text {corner }}\right) \bmod (1)=0$ is satisfied. This phase remains until the Wannier bands $v_{y}$ close the gap at $t=1.66$, followed by the reemergence of the type-II QTI. We also observe that the type-II AQTI reappears at $t=3.94$ as a result of the vanishing of the gap of $\mathrm{ES}_{y}$.

\section{EXPERIMENTAL REALIZATION}

These new phases can be observed through quench dynamics in cold atom experiments. In fact, Ref. [1] has introduced an experimental scheme to realize the type-I quadrupole model. In the scheme, laser beams are used to engineer a superlattice with four sites in each unit cell [see Fig. 1(a)]. Tunnelling along $y$ is suppressed by a linear potential. Then, Raman laser beams are applied to restore the hopping with a phase of $\pi$ per plaquette $[71,72]$. Initially, we can tune the superlattice to realize large barriers between unit cells, which suppress the tunneling between unit cells despite the presence of Raman lasers, realizing our initial Hamiltonian with almost zero $\lambda$. The cold atoms are prepared in the ground state of this Hamiltonian. After that, we suddenly change the model to our final Hamiltonian by tuning the superlattice and Raman lasers and perform the tomography of the evolving states. We can achieve the tomography by first removing atoms at two sites in each unit cell and then performing the tomography of the remaining sites by time-of-flight measurements $[73,74]$. The atoms at these sites can be kicked out of the trap by shining resonant laser beams to the sites to excite them to the $P$ state, which rapidly decays through spontaneous emission and escapes the trap. This is feasible given that current experiments can realize laser beams with the diameter as small as $600 \mathrm{~nm}$ [75,76], comparable to the lattice constant. With measured states, the quadrupole moments, entanglement spectrum, and edge polarization can be obtained.

In addition, we propose a scheme to simulate the Hamiltonian (1) in electric circuits to realize these new phases, as illustrated in Fig. 10. In fact, the Hamiltonians (20), (23), and (24) can be experimentally implemented using simplified electric networks. We note that the type-I QTI has already been observed in electric circuits [26]. In the circuits, a 


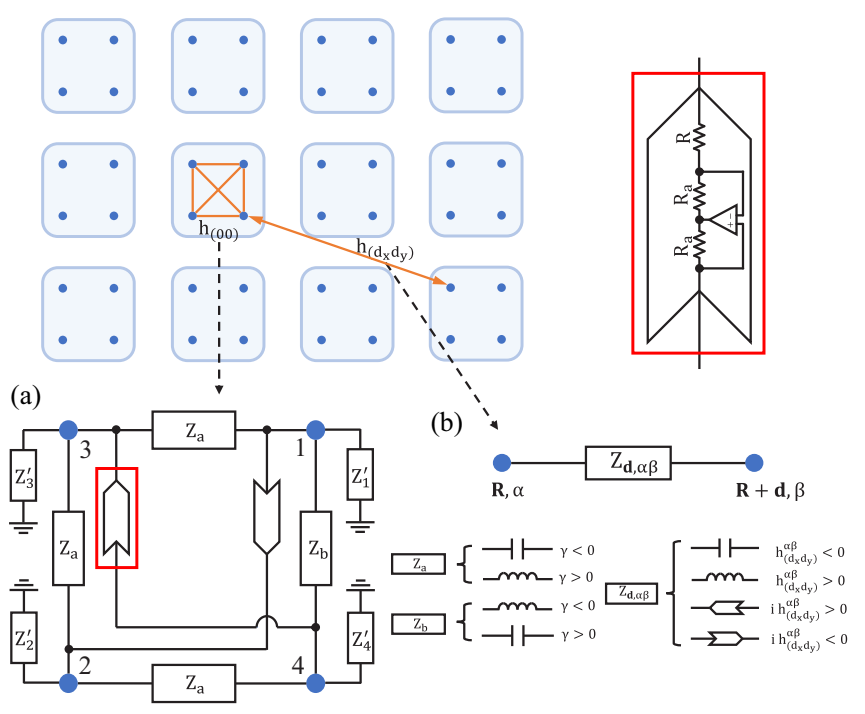

FIG. 10. A scheme to realize our Hamiltonian in electric circuits. (a) The electric device configuration to simulate the term $h_{(00)}$ within a unit cell. Here, the box labeled by $Z_{a}$ or $Z_{b}$ can be either a capacitor or a inductor depending on the sign of $\gamma$ with their impedances being $Z_{a}=-1 /(i \gamma)$ and $Z_{b}=1 /(i \gamma)$, respectively. The device in the red box denotes a negative impedance converter with current inversion (INIC), the impedance of which depends on the direction of the current. The resistance of the INIC should be taken as $R=1 / \Delta$. The boxes labeled by $Z_{a=1,2,3,4}^{\prime}$ represent device configurations to eliminate the unnecessary onsite terms (see Appendix H). (b) A suitable electric device is applied to connect two nodes: $(\mathbf{R}, \alpha)$ and $(\mathbf{R}+\mathbf{d}, \beta)$ for distinct unit cells. The device can be either a capacitor, or an inductor or an INIC with the impedance being $Z_{\mathbf{d}, \alpha \beta}=i / h_{\left(d_{x} d_{y}\right)}^{\alpha \beta}$, as shown in the lower right corner figure. Here, $\mathbf{d}=d_{x} \mathbf{e}_{x}+d_{y} \mathbf{e}_{y}$.

Hamiltonian is simulated by a Laplacian, a matrix connecting electric potentials with currents, i.e., $\mathbf{I}=J \mathbf{V}$, where $\mathbf{I}$ and $\mathbf{V}$ are column vectors, each entry of which denotes the corresponding current flowing into the corresponding node and the electric potential there, respectively [77]. Appropriate electric devices, such as capacitors, inductors and INICs [78,79], are applied to connect different nodes to mimic the hopping between different sites within or outside of a unit cell. The edge polarization and quadrupole moment can be obtained by measuring the single-point impedances of the circuit, and the existence of corner modes can be shown by measuring the resonance of two-point impedances near the corners, as detailed in Appendix H. Type-II QTIs may also be realized in other systems, such as solid-state materials, cold atoms and photonic crystals.

\section{CONCLUSION}

In summary, we discover a type of QTI violating an established classical relation. This relation is maintained in a quantum system as the Wannier band and edge energy spectrum close their gaps simultaneously. However, the appearance of the type-II QTI indicates that the two gaps do not necessarily vanish at the same time. We also find the anomalous quadrupole insulating phases that cannot be characterized by the previously introduced Wannier-sector polarizations. We introduce a new topological invariant for a Wilson line to characterize them for a system with reflection symmetries; such methods to characterize the topological property change for a Wannier band can also be applied to systems with other symmetries. In addition, we predict another topological phase with quantized edge polarizations but without zero-energy corner modes and quadrupole moments. Based on the type-II insulating phase, we find new pumping phenomena, leading to 3D higher-order topological insulators. We further show that these new topological phenomena in two dimensions can emerge from quench dynamics, which can be experimentally achieved in ultracold atomic gases. We also introduce an experimental proposal with electric circuits to simulate these new models. Our results demonstrate that new multipole topological insulators with exotic properties can exist beyond classical constraints, opening a new direction for exploring multipole topological insulators.

\section{ACKNOWLEDGMENTS}

We thank Q. Zeng, Y.-L. Tao, and D.-L. Deng for helpful discussions. We also thank B. J. Wieder for helpful discussions and bringing us Ref. [66], where the breakdown of the correspondence between Wannier and edge spectra was found in a system with neither particle-hole nor chiral symmetry. Y.-B.Y., K.L., and Y.X. are supported by the start-up fund from Tsinghua University, the National Thousand-YoungTalents Program and the National Natural Science Foundation of China (Grant No. 11974201). We acknowledge in addition support from the Frontier Science Center for Quantum Information of the Ministry of Education of China, Tsinghua University Initiative Scientific Research Program, and the National key Research and Development Program of China (Grant No. 2016YFA0301902).

\section{APPENDIX A: THE RELATIONSHIP BETWEEN TWO SIMPLEST INSULATORS AND THE TYPE-II AQTI}

In this Appendix, by relating two simplest insulators with zero and nonzero quadrupole moments to the type-II AQTI, we verify that the quadrupole moments obtained in the type-II AQTI are reasonable.

Let us first consider a topologically trivial model described by the Hamiltonian,

$$
H^{\text {trivial }}=\sum_{\mathbf{R}} \hat{c}_{\mathbf{R}}^{\dagger} h \hat{c}_{\mathbf{R}}
$$

where

$$
h=\left(\begin{array}{cc}
0 & -\sigma_{0}+i \sigma_{y} \\
\text { H.c. } & 0
\end{array}\right) .
$$

This model has four orbital degrees of freedom in each unit cell. There is no tunneling between distinct unit cells [see Fig. 11(a)], showing that it is a topologically trivial atomic insulator. Evidently, this model does not possess quadrupole moment at half filling since the positive charges and the electrons occupy the same position. We note that this trivial insulator corresponds to a trivial phase in the model in Ref. [1] with $\lambda_{x}=\lambda_{y}=0$ and $\gamma_{x}=-\gamma_{y}=-1$. 
(a)

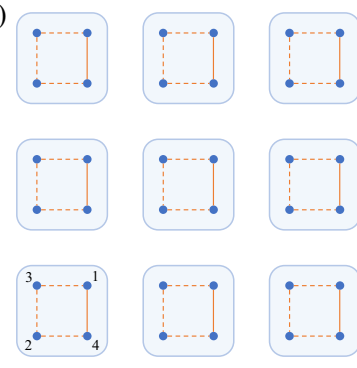

(b)

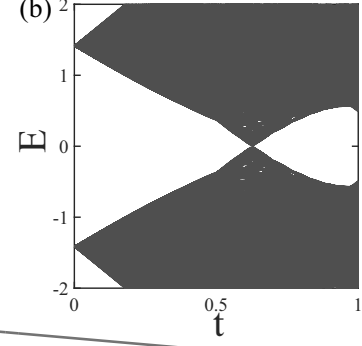

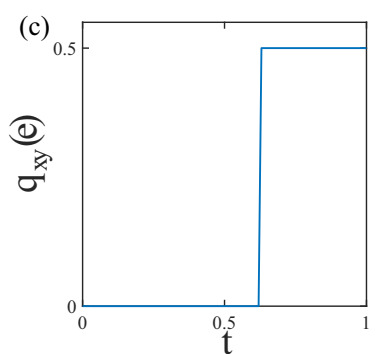

\section{Type-II AQTI}
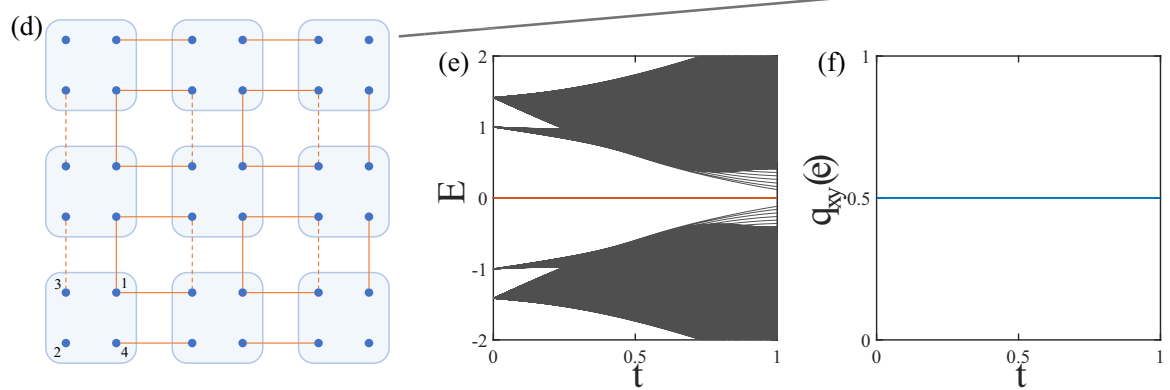

FIG. 11. The relationship between the type-II AQTI and two simplest models without and with quadruopole moments. (a) Schematics of a simplest model of a trivial insulator with zero quadrupole moments and (d) schematics of a simplest model of type-I QTI with quantized nonzero quadrupole moment. In (a) and (d), the solid and dashed red lines represent the hopping terms with positive and negative signs, respectively. The index $\alpha=1,2,3,4$ denotes the sites within a unit cell. (b) The energy spectrum (obtained under periodic boundary conditions along both $x$ and $y$ directions) and (c) the quadrupole moment as we change a system from the trivial insulating phase in a to a type-II anomalous quadrupole topological insulating phase. Here the quadrupole moment changes suddenly from zero to $e / 2$ when the bulk energy gap vanishes, implying that the type-II AQTI is topologically distinct from the trivial insulator. This also distinguishes the type-II AQTI from the trivial insulator attached with a pair of SSH models. (e) The energy spectrum (obtained under open boundary conditions along both $x$ and $y$ directions) and $\mathrm{f}$, the quadrupole moment as we change a system from the type-I QTI in (d) to a type-II AQTI. Here the quadrupole moment remains unchanged and both bulk and edge energy gaps remain open and the zero-energy corner states exist during the whole process. It shows that the type-II AQTI possesses the same quadrupole moment as the type-I QTI.

To see the connection between the trivial insulator and the type-II AQTI, we construct a model $H_{1}(t)$ parameterized by $t \in[0,1]$ as follows:

$$
H_{1}(t)= \begin{cases}(1-2 t) H^{\text {trivial }}+2 t H(\gamma=-1) & 0 \leqslant t<0.5, \\ H(\gamma=2.4 t-2.2) & 0.5 \leqslant t \leqslant 1,\end{cases}
$$

where $H$ denotes the Hamiltonian (1) in the main text. This Hamiltonian connects the trivial insulator for $t=0$ with the type-II AQTI for $t=1$ corresponding to the type-II AQTI marked by the green square in Fig. 2(c) in the main text.

In Figs. 11(b) and 11(c), we present the energy spectrum (obtained under periodic boundary conditions along both $x$ and $y$ directions) and the quadrupole moment as $t$ varies from 0 to 1 . We find that at an intermediate point, the bulk energy gap closes and simultaneously the quadrupole moment changes abruptly from 0 to $1 / 2$. This suggests that the typeII AQTI is topologically distinct from the trivial insulator with respect to the bulk property and this distinction can be characterized by the quadrupole moment. This also shows that the type-II AQTI is completely different from the trivial insulator attached with a pair of SSH models. Even though the edge polarizations are the same for these two models and zero-energy corner modes exist for both of them, their bulk topological properties are topologically different (the former has nonzero quadrupole moment while the latter has zero one).

To see the connection between the type-I QTI and the typeII AQTI, we construct another model $H_{2}(t)$ parameterized by $t \in[0,1]$ as follows:

$$
H_{2}(t)=(1-t) H^{\text {typeI }}+t H(\gamma=0.2) \text {. }
$$

Here

$$
H^{\text {typeI }}=\sum_{\mathbf{R}}\left[\hat{c}_{\mathbf{R}+\mathbf{e}_{x}}^{\dagger} h_{(10)}+\hat{c}_{\mathbf{R}+\mathbf{e}_{y}}^{\dagger} h_{(01)}\right] \hat{c}_{\mathbf{R}}+\text { H.c. },
$$

with

$$
h_{10}=\left(\begin{array}{llll}
0 & 0 & 0 & 0 \\
0 & 0 & 0 & 1 \\
1 & 0 & 0 & 0 \\
0 & 0 & 0 & 0
\end{array}\right) \text { and } h_{01}=\left(\begin{array}{rrrr}
0 & 0 & 0 & 0 \\
0 & 0 & -1 & 0 \\
0 & 0 & 0 & 0 \\
1 & 0 & 0 & 0
\end{array}\right)
$$

describes a typical QTI with only intercell hoppings [see Fig. 11(d)] corresponding to the model in Ref. [1] with $\gamma_{x}=$ $\gamma_{y}=0$ and $\lambda_{x}=\lambda_{y}=1$. This model has nonzero quadrupole moment, zero-energy corner modes leading to fractional corner charges at half filling and edge polarizations at all four 
boundaries [1] and thus belongs to the type-I QTI. $H_{2}(t)$ connects this type-I QTI with a type-II AQTI.

Figure 11(e) illustrates that both bulk and edge energy gaps (see the energy spectrum obtained under open boundary conditions along both $x$ and $y$ directions) remain open as we vary $t$ from 0 to 1 . During this process, the quadrupole moment also remains unchanged (equal to $e / 2$ ) [see Fig. 11(f)]. This suggests that the type-II AQTI shares the same bulk topological property as the type-I QTI characterized by the quadrupole moment. Their difference arises from the Wannier band gap closing, leading to different edge polarizations.

The above discussion suggests the validity of our calculation of the quadrupole moments in our model in the sense that the quadrupole moments change when the bulk energy gap closes when a system changes from a typical atomic insulator without quadrupole moments to a type-II AQTI. In addition, this moment remains unchanged when we change a system from a typical QTI with nonzero quadrupole moments to a type-II AQTI. This confirms that the type-II AQTI is a new type of qudrupole topological insulating phase.

\section{APPENDIX B: THE HAMILTONIAN IN MOMENTUM SPACE}

In this Appendix, we write down the explicit form of our Hamiltonian in momentum space as

$$
H(\mathbf{k})=\sum_{i, j=0}^{3} g_{i j}(\mathbf{k}) \tau_{i} \otimes \sigma_{j},
$$

where all the nonzero $g_{i j}$ s are given by

$$
\begin{gathered}
g_{01}=2 t_{2} \sin \left(2 k_{x}\right), \\
g_{03}=-4 t_{2} \cos \left(k_{x}\right) \sin \left(k_{y}\right), \\
g_{10}=\gamma+2 t_{1} \cos \left(k_{x}\right)+2 t_{1}^{\prime} \cos \left(k_{y}\right)+4 t_{2} \cos \left(k_{x}\right) \cos \left(k_{y}\right) \\
-4 t_{2}^{\prime} \cos \left(2 k_{x}\right) \cos \left(k_{y}\right), \\
g_{21}=-2 t_{1} \sin \left(k_{y}\right)-2 t_{2} \sin \left(2 k_{y}\right)-4 t_{2}^{\prime} \cos \left(k_{x}\right) \sin \left(k_{y}\right) \\
+4 t_{2}^{\prime} \cos \left(k_{x}\right) \sin \left(2 k_{y}\right), \\
g_{22}=\gamma-2 t_{1} \cos \left(k_{y}\right)-2 t_{2} \cos \left(2 k_{y}\right)-4 t_{2}^{\prime} \cos \left(k_{x}\right) \cos \left(k_{y}\right) \\
+4 t_{2}^{\prime} \cos \left(k_{x}\right) \cos \left(2 k_{y}\right), \\
g_{23}=-2 t_{1} \sin \left(k_{x}\right)-4 t_{2} \sin \left(k_{x}\right) \cos \left(k_{y}\right) \\
+4 t_{2}^{\prime} \sin \left(2 k_{x}\right) \cos \left(k_{y}\right), \\
g_{31}=-4 t_{2} \cos \left(k_{x}\right) \sin \left(k_{y}\right)-2 t_{2}^{\prime} \sin \left(2 k_{y}\right), \\
g_{32}=\Delta+2 t_{1}^{\prime} \cos \left(k_{x}\right)+2 t_{2} \cos \left(2 k_{x}\right)-2 t_{2}^{\prime} \cos \left(2 k_{y}\right) \\
-4 t_{2} \cos \left(k_{x}\right) \cos \left(k_{y}\right), \\
g_{33}=-2 t_{2} \sin \left(2 k_{x}\right) .
\end{gathered}
$$

One can easily check that this Hamiltonian respects the reflection symmetry: $\hat{m}_{x} H\left(k_{x}, k_{y}\right) \hat{m}_{x}^{-1}=H\left(-k_{x}, k_{y}\right)$ with $\hat{m}_{x}=\tau_{1} \otimes \sigma_{3}, \quad \hat{m}_{y} H\left(k_{x}, k_{y}\right) \hat{m}_{y}^{-1}=H\left(k_{x},-k_{y}\right) \quad$ with $\quad \hat{m}_{y}=$ $\tau_{1} \otimes \sigma_{1}$, and the particle-hole symmetry: $\Xi H\left(k_{x}, k_{y}\right) \Xi^{-1}=$ $-H\left(-k_{x},-k_{y}\right)$ with $\Xi=\tau_{3} \otimes \sigma_{0} \kappa$.

\section{APPENDIX C: TOPOLOGICALLY TRIVIAL PHASE}

In this Appendix, we display richer physics in the topologically trivial phase as shown in Fig. 12. We find four regions corresponding to distinct $N_{v_{\lambda}}^{0}(\lambda=x, y):\left(N_{v_{x}}^{0}, N_{v_{v}}^{0}\right)=$ $(0,0),(4,0),(2,0),(2,2)$, even though they all have zero quadrupole moments and edge polarizations. This is reasonable as the edge states of the Wannier spectrum with zero Wannier centers do not contribute to the dipole moments. Despite being topologically trivial, these phases can have nonzero Wannier-sector polarization when odd number of pairs of edge states of the Wannier spectrum exists with zero eigenvalues. In particular, in the region with $\left(N_{v_{x}}^{0}, N_{v_{v}}^{0}\right)=(2,2)$, although both Wannier-sector polarizations are nonzero, i.e., $\left(p_{y}^{v_{x}}, p_{x}^{v_{y}}\right)=(1 / 2,1 / 2)$, the quadrupole moment and edge polarizations do not exist, implying the breakdown of the Wannier-sector polarizations to characterize the edge polarization and the quadrupole moments.

\section{APPENDIX D: A TOPOLOGICAL INVARIANT FOR A WILSON LINE}

In this Appendix, we will derive a topological invariant for a Wilson line characterizing the change of the topological property of the Wannier band.

\section{Generic symmetry constraint of a Wilson line}

Let us consider a generic symmorphic lattice symmetry: $\mathbf{r} \rightarrow D_{g} \mathbf{r}$, described by

$$
g H(\mathbf{k}) g^{-1}=H\left(D_{g} \mathbf{k}\right),
$$

where $g$ is a representation of the symmetric operation in momentum space, which is unitary. If $\left|u_{\mathbf{k}}^{n}\right\rangle$ is an eigenstate of $H(\mathbf{k})$ corresponding to the eigenenergy $E_{\mathbf{k}}^{n}$, then $g\left|u_{\mathbf{k}}^{n}\right\rangle$ is an eigenstate of $H\left(D_{g} \mathbf{k}\right)$ corresponding to the same energy. As a result, we can write $g\left|u_{\mathbf{k}}^{n}\right\rangle$ in terms of eigenstates of $H\left(D_{g} \mathbf{k}\right)$,

$$
g\left|u_{\mathbf{k}}^{n}\right\rangle=\sum_{m=1}^{N_{\text {occ }}}\left|u_{D_{g} \mathbf{k}}^{m}\right|\left\langle u_{D_{g} \mathbf{k}}^{m}|g| u_{\mathbf{k}}^{n}\right\rangle=\sum_{m=1}^{N_{\text {occ }}}\left|u_{D_{g} \mathbf{k}}^{m}\right| B_{g, \mathbf{k}}^{m n},
$$

where $B_{g, \mathbf{k}}^{m n}=\left\langle u_{D_{g} \mathbf{k}}^{m}|g| u_{\mathbf{k}}^{n}\right\rangle$ is a unitary sewing matrix that connects states at $\mathbf{k}$ with states at $D_{g} \mathbf{k}$. Since we are interested in the occupied bands, the superscript only enumerates the occupied states from 1 to the total number of the occupied states $N_{\text {occ }}$.

We now define the Wilson line following a path $C$ in momentum space from $\mathbf{k}_{i}$ to $\mathbf{k}_{f}$ as

$$
\mathcal{W}_{C, \mathbf{k}_{f} \leftarrow \mathbf{k}_{i}}=F_{\mathbf{k}_{f}-\Delta \mathbf{k}_{N-1}} \cdots F_{\mathbf{k}_{i}+\Delta \mathbf{k}_{1}} F_{\mathbf{k}_{i}}
$$

where $\left[F_{\mathbf{k}_{j^{\prime}}}\right]^{m n}=\left\langle u_{\mathbf{k}_{j^{\prime}}+\Delta \mathbf{k}_{j^{\prime}+1}}^{m} \mid u_{\mathbf{k}_{j^{\prime}}}^{n}\right\rangle$ with $m$ and $n$ being the indices for the occupied bands and $\Delta \mathbf{k}_{j}$ dividing the trajectory into $N$ segments and $j^{\prime}=0,1, \ldots, N-1$ and $\mathbf{k}_{j^{\prime}}=\mathbf{k}_{i}+$ $\sum_{j=1}^{j^{\prime}} \Delta \mathbf{k}_{j}$. In the limit $N \rightarrow \infty$, we can write the Wilson line 


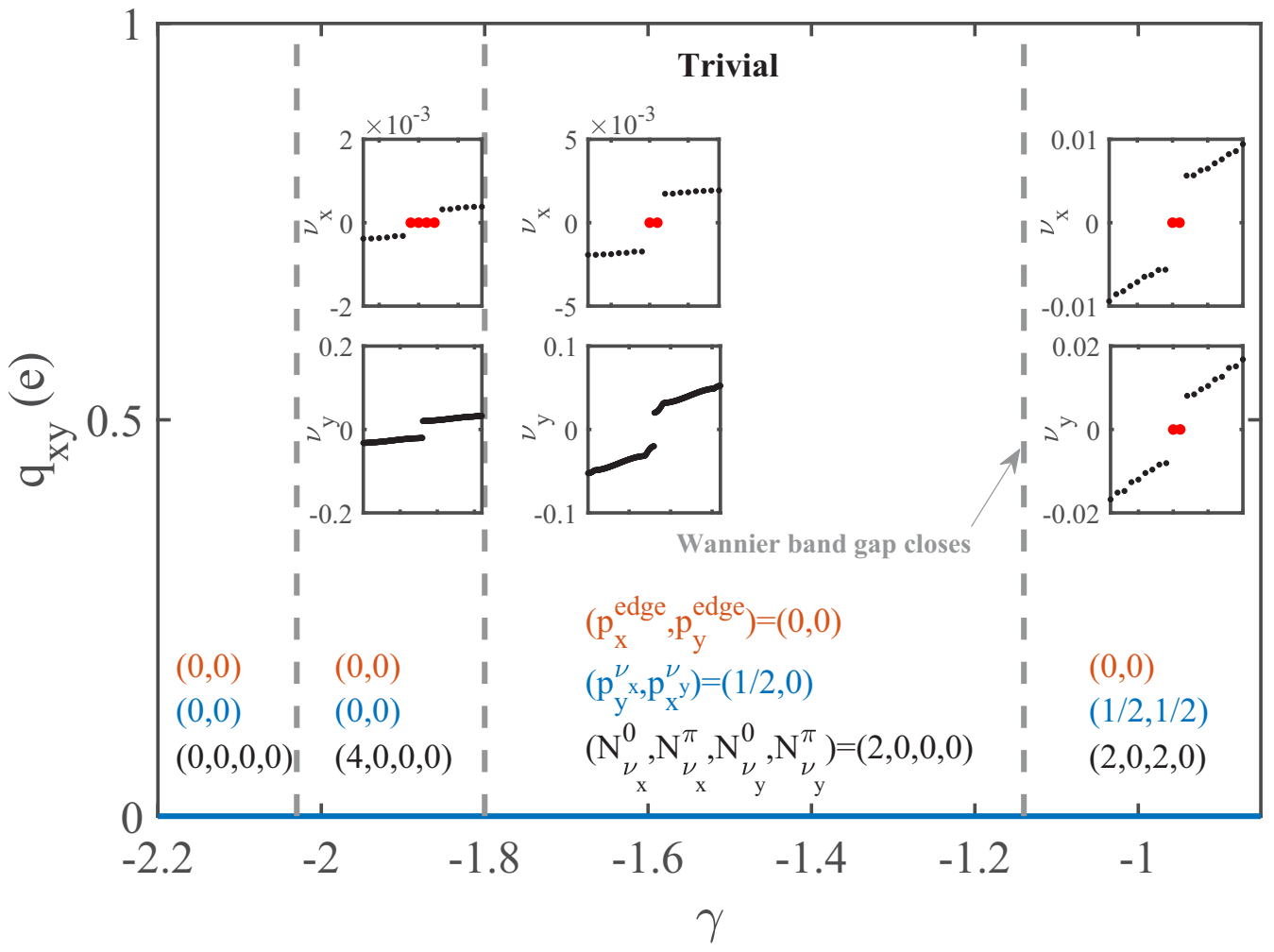

FIG. 12. Distinct regions in topologically trivial insulators. The subsets display the Wannier spectrum $v_{x}\left(v_{y}\right)$ for periodic boundary conditions along $x(y)$ and open ones along $y(x)$ with the isolated Wannier centers highlighted by red circles. The edge polarization $\left(p_{x}^{\text {edge }}, p_{y}^{\text {edge }}\right)$, the Wannier-sector polarization $\left(p_{y}^{v_{x}}, p_{x}^{v_{y}}\right)$, the number of edge states of the Wannier Hamiltonian $N_{v} \equiv\left(N_{v_{x}}^{0}, N_{v_{x}}^{\pi}, N_{v_{y}}^{0}, N_{v_{y}}^{\pi}\right)$ are also shown. The vertical dashed lines represent the critical points where the Wannier spectrum gap closes.

in the following compact form,

$$
\begin{aligned}
\mathcal{W}_{C, \mathbf{k}_{f} \leftarrow \mathbf{k}_{i}} & =\lim _{N \rightarrow \infty} \prod_{n=1}^{N-1}\left(I-i \Delta \mathbf{k}_{n} \cdot \mathcal{A}_{\mathbf{k}_{n}}\right) \\
& =\overline{\exp }\left(-i \int_{\mathbf{k}_{i}}^{\mathbf{k}_{f}} \mathcal{A}_{\mathbf{k}} \cdot d \mathbf{k}\right),
\end{aligned}
$$

where $\overline{\exp }(\cdots)$ denotes the path-ordered exponential, and $\left[\mathcal{A}_{\mathbf{k}}\right]^{m n}=-i\left\langle u_{\mathbf{k}}^{m} \mid \partial_{\mathbf{k}} u_{\mathbf{k}}^{n}\right\rangle$ is the non-Abelian Berry connection. Since $\mathcal{A}_{\mathbf{k}}$ is a Hermitian matrix, the Wilson loop is unitary, reminiscent of a time evolution operator.

Since $\left|u_{\mathbf{k}}^{n}\right\rangle=g^{\dagger} \sum_{m}\left|u_{D_{g} \mathbf{k}}^{m}\right\rangle B_{g, \mathbf{k}}^{m n}$, we have

$$
\left[F_{\mathbf{k}_{j}}\right]^{m n}=\sum_{m^{\prime}, n^{\prime}}\left(B_{g, \mathbf{k}_{j+1}}^{\dagger}\right)^{m m^{\prime}}\left\langle u_{D_{g} \mathbf{k}_{j+1}}^{m^{\prime}} \mid u_{D_{g} \mathbf{k}_{j}}^{n^{\prime}}\right\rangle B_{g, \mathbf{k}_{j}}^{n^{\prime} n},
$$

leading to

$$
\mathcal{W}_{C, \mathbf{k}_{f} \leftarrow \mathbf{k}_{i}}=B_{g, \mathbf{k}_{f}}^{\dagger} \mathcal{W}_{D_{g} C, D_{g} \mathbf{k}_{f} \leftarrow D_{g} \mathbf{k}_{i}} B_{g, \mathbf{k}_{i}},
$$

which can be equivalently expressed as

$$
B_{g, \mathbf{k}_{f}} \mathcal{W}_{C, \mathbf{k}_{f} \leftarrow \mathbf{k}_{i}} B_{g, \mathbf{k}_{i}}^{\dagger}=\mathcal{W}_{D_{g} C, D_{g} \mathbf{k}_{f} \leftarrow D_{g} \mathbf{k}_{i}}=\mathcal{W}_{D_{g} \mathbf{k}_{f} \leftarrow D_{g} \mathbf{k}_{i}},
$$

where $D_{g} C$ denotes a new path obtained by applying the symmetry operation on the original path $C$ (we will skip this notation for simplicity).

\section{Reflection symmetry constraint and topological invariants}

We now consider the reflection symmetry $M_{x}$, which, based on Eq. (D7), gives

$$
B_{m_{x}, \mathbf{k}_{f}} \mathcal{W}_{x, \mathbf{k}} B_{m_{x}, \mathbf{k}_{i}}^{\dagger}=\mathcal{W}_{x,\left(-k_{x}-2 \pi, k_{y}\right) \leftarrow\left(-k_{x}, k_{y}\right)}=\mathcal{W}_{-x, M_{x} \mathbf{k}},
$$

where $\mathcal{W}_{x, \mathbf{k}}$ and $\mathcal{W}_{-x, \mathbf{k}}$ are defined as

$$
\begin{gathered}
\mathcal{W}_{x, \mathbf{k}}=\mathcal{W}_{\left(k_{x}+2 \pi, k_{y}\right) \leftarrow\left(k_{x}, k_{y}\right)}, \\
\mathcal{W}_{-x, \mathbf{k}}=\mathcal{W}_{\left(k_{x}-2 \pi, k_{y}\right) \leftarrow\left(k_{x}, k_{y}\right)},
\end{gathered}
$$

with $x$ and $-x$ labeling the direction that a Wilson loop is obtained. Since the Wilson loop is unitary, we can write it as

$$
\mathcal{W}_{x, \mathbf{k}}^{(0)}=\mathcal{W}_{x,\left(0, k_{y}\right)}=e^{i H_{\mathcal{W}_{x}}^{(0)}\left(k_{y}\right)},
$$

where $H_{\mathcal{W}_{x}}^{(0)}\left(k_{y}\right)=-i \log \left(\mathcal{W}_{x, \mathbf{k}}^{(0)}\right)$ is the Wannier Hamiltonian for $k_{x}=0$. Given that the Wannier Hamiltonian is a multivalued function of the Wilson loop, we redefine it with respect to a logarithm branch cut $\epsilon$,

$$
H_{\mathcal{W}_{x}}^{\epsilon}\left(k_{y}\right)=-i \log _{\epsilon}\left(\mathcal{W}_{x, \mathbf{k}}^{(0)}\right),
$$

where we take $\log _{\epsilon} e^{i \phi}=i \phi$, for $\epsilon \leqslant \phi<\epsilon+2 \pi$.

Now we can obtain the symmetry constraint on the Wannier Hamiltonian,

$$
\begin{aligned}
B_{m_{x},\left(0, k_{y}\right)} H_{\mathcal{W}_{x}}^{\epsilon}\left(k_{y}\right) B_{m_{x},\left(0, k_{y}\right)}^{\dagger} & =-H_{\mathcal{W}_{x}}^{-\epsilon}\left(k_{y}\right)+2 \pi I_{N_{\mathrm{occ}}} \\
& =-H_{\mathcal{W}_{x}^{-\epsilon+2 \pi}}\left(k_{y}\right)+4 \pi I_{N_{\mathrm{occ}}} .
\end{aligned}
$$




$$
\begin{aligned}
& \text { Proof. } \\
& B_{m_{x},\left(0, k_{y}\right)} H_{\mathcal{W}_{x}}^{\epsilon}\left(k_{y}\right) B_{m_{x},\left(0, k_{y}\right)}^{\dagger} \\
& =-i B_{m_{x},\left(0, k_{y}\right)} \log _{\epsilon}\left(\mathcal{W}_{x, \mathbf{k}}^{(0)}\right) B_{m_{x},\left(0, k_{y}\right)}^{\dagger} \\
& =-i \log _{\epsilon}\left[B_{m_{x},\left(0, k_{y}\right)} \mathcal{W}_{x, \mathbf{k}}^{(0)} B_{m_{x},\left(0, k_{y}\right)}^{\dagger}\right] \\
& =-i \log _{\epsilon}\left[\mathcal{W}_{-x, \mathbf{k}}^{(0)}\right] \\
& =-i \log _{\epsilon}\left[\left(\mathcal{W}_{x, \mathbf{k}}^{(0)}\right)^{-1}\right] \\
& =-i \sum_{n} \log _{\epsilon}\left(e^{-i v_{x}^{(n)}}\right)\left|v_{x}^{(n)}\right\rangle\left\langle v_{x}^{(n)}\right| \\
& =-i \sum_{n}\left[-\log _{-\epsilon}\left(e^{i v_{x}^{(n)}}\right)+2 \pi i\right]\left|v_{x}^{(n)}\right\rangle\left\langle v_{x}^{(n)}\right| \\
& =-H_{\mathcal{W}_{x}}^{-\epsilon}\left(k_{y}\right)+2 \pi I_{N_{\text {occ }}} \\
& =-i \sum_{n}\left[-\log _{-\epsilon+2 \pi}\left(e^{i v_{x}^{(n)}}\right)+4 \pi i\right]\left|v_{x}^{(n)}\right\rangle\left\langle v_{x}^{(n)}\right| \\
& =-H_{\mathcal{W}_{x}}^{-\epsilon+2 \pi}\left(k_{y}\right)+4 \pi I_{N_{\text {occ }}},
\end{aligned}
$$

where $\left|v_{x}^{(n)}\right\rangle$ is the $n$th eigenvector of the Wilson loop $\mathcal{W}_{x, \mathbf{k}}^{(0)}$ corresponding to the eigenvalue $e^{i v_{x}^{(n)}}$. In the derivation, we have also used the following relations:

$$
\begin{aligned}
& \log _{\epsilon}\left(e^{-i \phi}\right)=-\log _{-\epsilon}\left(e^{i \phi}\right)+2 \pi i, \\
& \log _{-\epsilon}\left(e^{i \phi}\right)=\log _{-\epsilon+2 \pi}\left(e^{i \phi}\right)-2 \pi i .
\end{aligned}
$$

We now define the Wilson line with respect to $\epsilon$ by

$$
\mathcal{W}_{k_{x} \leftarrow 0}^{\epsilon}\left(k_{y}\right) \equiv \mathcal{W}_{k_{x} \leftarrow 0}\left(k_{y}\right) \exp \left[-i H_{W_{x}}^{\epsilon}\left(k_{y}\right) \frac{k_{x}}{2 \pi}\right]
$$

where $\mathcal{W}_{k_{x} \leftarrow 0}\left(k_{y}\right) \equiv \mathcal{W}_{\left(k_{x}, k_{y}\right) \leftarrow\left(0, k_{y}\right)}$. It can be easily checked that $\mathcal{W}_{2 \pi \leftarrow 0}^{\epsilon}\left(k_{y}\right)=I_{N_{\text {occ }}}$, implying that $\mathcal{W}_{k_{x} \leftarrow 0}^{\epsilon}\left(k_{y}\right)$ is periodic with respect to $k_{x}$.

In the following, we will prove the symmetry constraints on the Wilson line with respect to $\epsilon$,

$$
\begin{aligned}
B_{m_{x},\left(k_{x}, k_{y}\right)} \mathcal{W}_{k_{x} \leftarrow 0}^{\epsilon}\left(k_{y}\right) B_{m_{x},\left(0, k_{y}\right)}^{\dagger} & =\mathcal{W}_{-k_{x}+2 \pi \leftarrow 0}^{-\epsilon}\left(k_{y}\right) \exp \left(-i k_{x}\right) \\
& =\mathcal{W}_{-k_{x}+2 \pi \leftarrow 0}^{-\epsilon+2 \pi}\left(k_{y}\right) \exp \left(-2 i k_{x}\right) .
\end{aligned}
$$

Proof. We can directly obtain

$$
\begin{aligned}
B_{m_{x},\left(k_{x}, k_{y}\right)} \mathcal{W}_{k_{x} \leftarrow 0}^{\epsilon}\left(k_{y}\right) B_{m_{x},\left(0, k_{y}\right)}^{\dagger} \\
=B_{m_{x},\left(k_{x}, k_{y}\right)} \mathcal{W}_{k_{x} \leftarrow 0}\left(k_{y}\right) B_{m_{x},\left(0, k_{y}\right)}^{\dagger} B_{m_{x},\left(0, k_{y}\right)} \\
\quad \times e^{-i H_{W_{x}}^{\epsilon}\left(k_{y}\right) \frac{k_{x}}{2 \pi}} B_{m_{x},\left(0, k_{y}\right)}^{\dagger}, \\
=\mathcal{W}_{-k_{x} \leftarrow 0}\left(k_{y}\right) e^{i H_{W_{x}}^{-\epsilon}\left(k_{y}\right) \frac{k_{x}}{2 \pi}} e^{-i k_{x}}=\mathcal{W}_{-k_{x} \leftarrow 0}^{-\epsilon} e^{-i k_{x}}, \quad(\mathrm{D} 21) \\
=\mathcal{W}_{-k_{x} \leftarrow 0}\left(k_{y}\right) e^{i H_{W_{x}}^{-\epsilon+2 \pi}\left(k_{y}\right) \frac{k_{x}}{2 \pi}} e^{-2 i k_{x}}=\mathcal{W}_{-k_{x} \leftarrow 0}^{-\epsilon+2 \pi} e^{-2 i k_{x}} .
\end{aligned}
$$

With the aid of the following equation:

$$
\begin{aligned}
\mathcal{W}_{k_{x}+2 \pi \leftarrow 0}^{\epsilon}\left(k_{y}\right)= & \mathcal{W}_{k_{x}+2 \pi \leftarrow 2 \pi}\left[\mathcal{W}_{2 \pi \leftarrow 0}\left(k_{y}\right) \exp \left(-i H_{W_{x}}^{\epsilon}\right)\right] \\
& \times \exp \left(-i H_{W_{x}}^{\epsilon} \frac{k_{x}}{2 \pi}\right), \\
= & \mathcal{W}_{k_{x} \leftarrow 0}\left(k_{y}\right) \exp \left(-i H_{W_{x}}^{\epsilon} \frac{k_{x}}{2 \pi}\right), \\
= & \mathcal{W}_{k_{x} \leftarrow 0}^{\epsilon}\left(k_{y}\right),
\end{aligned}
$$

we obtain Eq. (D19).

At $k_{x}=\pi, \epsilon=0$ or $\epsilon=\pi$, Eq. (D19) leads to

$$
\begin{gathered}
B_{m_{x},\left(\pi, k_{y}\right)} \mathcal{W}_{\pi \leftarrow 0}^{\epsilon=0}\left(k_{y}\right) B_{m_{x},\left(0, k_{y}\right)}^{\dagger}=-\mathcal{W}_{\pi \leftarrow 0}^{\epsilon=0}\left(k_{y}\right), \\
B_{m_{x},\left(\pi, k_{y}\right)} \mathcal{W}_{\pi \leftarrow 0}^{\epsilon=\pi}\left(k_{y}\right) B_{m_{x},\left(0, k_{y}\right)}^{\dagger}=\mathcal{W}_{\pi \leftarrow 0}^{\epsilon=\pi}\left(k_{y}\right) .
\end{gathered}
$$

Let us present a theorem, showing that $B_{m_{x},\left(\pi, k_{y}\right)}=$ $B_{m_{x},\left(0, k_{y}\right)}$ in a specific basis.

Theorem D.1. Consider a Hamiltonian $H(\mathbf{k})$ describing an insulator at half filling. Suppose it respects reflection symmetry such that $\hat{m}_{x} H(\mathbf{k}) \hat{m}_{x}^{\dagger}=H\left(-k_{x}, k_{y}\right)$ and $\hat{m}_{y} H(\mathbf{k}) \hat{m}_{y}^{\dagger}=$ $H\left(k_{x},-k_{y}\right)$ with $\hat{m}_{x}$ and $\hat{m}_{y}$ anticommuting with each other. There exists a basis in which the sewing matrix takes the form

$$
B_{m_{x},\left(\pi, k_{y}\right)}=B_{m_{x},\left(0, k_{y}\right)}=\left(\begin{array}{cc}
I_{N_{\mathrm{occ}} / 2} & 0 \\
0 & -I_{N_{\mathrm{occ}} / 2}
\end{array}\right) .
$$

Proof. From definition of the sewing matrix, we have

$$
\begin{aligned}
& B_{m_{x},\left(0, k_{y}\right)}^{m n}=\left\langle u_{\left(0, k_{y}\right)}^{m}\left|\hat{m}_{x}\right| u_{\left(0, k_{y}\right)}^{n}\right\rangle, \\
& B_{m_{x},\left(\pi, k_{y}\right)}^{m n}=\left\langle u_{\left(\pi, k_{y}\right)}^{m}\left|\hat{m}_{x}\right| u_{\left(\pi, k_{y}\right)}^{n}\right\rangle .
\end{aligned}
$$

Because $\hat{m}_{x}^{2}=1$, there are two eigenvalues: $\lambda= \pm 1$ for $\hat{m}_{x}$. If $\left|m_{\lambda}\right\rangle$ is an eigenvector of $\hat{m}_{x}$ corresponding to an eigenvalue $\lambda$, then $\hat{m}_{y}\left|m_{\lambda}\right\rangle$ is another eigenvector of $\hat{m}_{x}$ with an eigenvalue being $-\lambda$, since $\hat{m}_{x} \hat{m}_{y}\left|m_{\lambda}\right\rangle=-\hat{m}_{y} \hat{m}_{x}\left|m_{\lambda}\right\rangle=-\lambda \hat{m}_{y}\left|m_{\lambda}\right\rangle$ arising from the anticommutation relation of $\hat{m}_{x}$ and $\hat{m}_{y}$, i.e., $\left\{\hat{m}_{x}, \hat{m}_{y}\right\}=0$. In addition, if two eigenvectors $\left|m_{\lambda}^{1}\right\rangle$ and $\left|m_{\lambda}^{2}\right\rangle$ are orthogonal, i.e., $\left\langle m_{\lambda}^{2} \mid m_{\lambda}^{1}\right\rangle=0$, then $\left\langle m_{\lambda}^{2}\left|\hat{m}_{y}^{\dagger} \hat{m}_{y}\right| m_{\lambda}^{1}\right\rangle=0$. This tells us that the eigenvectors of $\hat{m}_{x}$ come in pairs with eigenvalues \pm 1 .

Let us choose a basis $\beta=\beta_{1} \cup \beta_{-1}$ consisting of eigenvectors of $\hat{m}_{x}, \beta_{1}=\left\{\left|m_{1}^{1}\right\rangle, \ldots,\left|m_{1}^{N_{\text {occ }}}\right\rangle\right\}$ and $\beta_{-1}=$ $\left\{\hat{m}_{y}\left|m_{1}^{1}\right\rangle, \ldots, \hat{m}_{y}\left|m_{1}^{N_{\text {occ }}}\right\rangle\right\}$, where $N_{\text {occ }}$ is the number of occupied states at half filling for a fixed momentum. Because of the reflection symmetry along $x$ respected by the Hamiltonian, at $k_{x}=0$ or $\pi$, the Hamiltonian commutes with $\hat{m}_{x}$, i.e., $\left[H\left(k_{x}=\right.\right.$ $\left.\left.0, k_{y}\right), \hat{m}_{x}\right]=0$. As a result, in the basis $\beta, H\left(k_{x}=0, k_{y}\right)$ takes the following block form:

$$
H\left(k_{x}=0, k_{y}\right)=\left[\begin{array}{cc}
H_{1}\left(k_{y}\right) & 0 \\
0 & H_{-1}\left(k_{y}\right)
\end{array}\right],
$$

where $H_{\lambda}$ with $\lambda= \pm 1$ are $N_{\text {occ }} \times N_{\text {occ }}$ matrices.

If $\left|u_{1}\left(k_{y}=0\right)\right\rangle$ is an eigenstate of $H_{1}\left(k_{y}=0\right)$ corresponding to an eigenenergy $E_{1}\left(k_{y}=0\right)$, then $\hat{m}_{y} \mid u_{1}\left(k_{y}=\right.$ $0)\rangle$ is an eigenstate of $H_{-1}\left(k_{y}=0\right)$ with the same energy because $H \hat{m}_{y}\left|u_{1}\left(k_{y}=0\right)\right\rangle=E_{1}\left(k_{y}=0\right) \hat{m}_{y}\left|u_{1}\left(k_{y}=0\right)\right\rangle$ and $\hat{m}_{y}\left|u_{1}\left(k_{y}=0\right)\right\rangle \in \operatorname{span}\left\{\beta_{-1}\right\}$. This tells us that, at half filling, only half of eigenstates of $H_{1}$ and $H_{-1}$ are occupied. Consider an occupied basis $\beta_{\text {occ }}=\beta_{\text {occ }, 1} \cup \beta_{\text {occ, }-1}$ 
with $\beta_{\mathrm{occ}, \lambda}=\left\{\left|u_{\lambda,\left(k_{x}=0, k_{y}=0\right)}^{1}\right\rangle, \ldots,\left|u_{\lambda,\left(k_{x}=0, k_{y}=0\right)}^{N_{\mathrm{occ}} / 2}\right\rangle\right\}$ with $\lambda=$ \pm 1 , where $\left|u_{\lambda,\left(k_{x}=0, k_{y}=0\right)}^{j}\right\rangle$ with $j=1, \cdots, N_{\text {occ }} / 2$ being eigenstates of $H_{\lambda}\left(k_{x}=0, k_{y}=0\right)$. In this basis,

$$
B_{m_{x},(0,0)}=\left(\begin{array}{cc}
I_{N_{\mathrm{occ}} / 2} & 0 \\
0 & -I_{N_{\mathrm{occ}} / 2}
\end{array}\right),
$$

where $I_{N_{\text {occ }} / 2}$ is a $N_{\text {occ }} / 2 \times N_{\text {occ }} / 2$ identity matrix. For nonzero $k_{y}$, the number of occupied states in $H_{1}$ or $H_{-1}$ should remain unchanged; otherwise, the Hamiltonian would not be an insulator. For example, if $\left|u_{\lambda,\left(k_{x}=0, k_{y}=0\right)}^{1}\right\rangle$ becomes unoccupied as we continuously vary $k_{y}$, then its energy must intersect with the Fermi level, leading to a metallic phase instead of an insulating phase. This tells us that $B_{m_{x},\left(k_{x}=0, \pi, k_{y}\right)}$ takes the same form as in Eq. (D32) in the ba$\operatorname{sis} \beta_{\mathrm{occ}, \lambda}=\left\{\left|u_{\lambda,\left(k_{x}=0, \pi, k_{y}\right)}^{1}\right\rangle, \ldots,\left|u_{\lambda,\left(k_{x}=0, \pi, k_{y}\right)}^{N_{\mathrm{occ}} / 2}\right\rangle\right\}$ with $\lambda= \pm 1$, where $\left|u_{\lambda,\left(k_{x}=0, \pi, k_{y}\right)}^{j}\right\rangle$ with $j=1, \ldots, N_{\text {occ }} / 2$ being eigenstates of $H_{\lambda}\left(k_{x}=0, \pi, k_{y}\right)$, respectively.

Based on the above theorem, we can write Eq. (D26) and Eq. (D27) into

$$
\begin{gathered}
S \mathcal{W}_{\pi \leftarrow 0}^{\epsilon=0}\left(k_{y}\right) S^{\dagger}=-\mathcal{W}_{\pi \leftarrow 0}^{\epsilon=0}\left(k_{y}\right), \\
S \mathcal{W}_{\pi \leftarrow 0}^{\epsilon=\pi}\left(k_{y}\right) S^{\dagger}=\mathcal{W}_{\pi \leftarrow 0}^{\epsilon=\pi}\left(k_{y}\right),
\end{gathered}
$$

where

$$
S=\left(\begin{array}{cc}
I_{N_{\mathrm{occ}} / 2} & 0 \\
0 & -I_{N_{\mathrm{occ}} / 2}
\end{array}\right)
$$

As a result,

$$
\begin{aligned}
\mathcal{W}_{\pi \leftarrow 0}^{\epsilon=0}\left(k_{y}\right)= & {\left[\begin{array}{cc}
0 & U_{+}^{\epsilon=0}\left(k_{y}\right) \\
U_{-}^{\epsilon=0}\left(k_{y}\right) & 0
\end{array}\right] \quad \begin{array}{cc}
\text { This gives us the transformation for } \\
0, \pi,
\end{array} } \\
& \mathcal{W}_{\pi \leftarrow 0}^{\epsilon=0}\left(k_{y}\right) \rightarrow\left\{\begin{array}{cc}
0 & U_{+}^{\epsilon=0}\left(k_{y}\right) e^{i\left[\theta_{-1}\left(0, k_{y}\right)-\theta_{+1}\left(\pi, k_{y}\right)\right]} \\
U_{-}^{\epsilon=0}\left(k_{y}\right) e^{i\left[\theta_{+1}\left(0, k_{y}\right)-\theta_{-1}\left(\pi, k_{y}\right)\right]} & 0
\end{array}\right\} \\
& \mathcal{W}_{\pi \leftarrow 0}^{\epsilon=\pi}\left(k_{y}\right) \rightarrow\left\{\begin{array}{cc}
U_{+}^{\epsilon=\pi}\left(k_{y}\right) e^{\left[\theta_{+1}\left(0, k_{y}\right)-\theta_{+1}\left(\pi, k_{y}\right)\right]} & 0 \\
0 & U_{-}^{\epsilon=\pi}\left(k_{y}\right) e^{i\left[\theta_{-1}\left(0, k_{y}\right)-\theta_{-1}\left(\pi, k_{y}\right)\right]}
\end{array}\right\} .
\end{aligned}
$$

$$
\mathcal{W}_{\pi \leftarrow 0}^{\epsilon=\pi}\left(k_{y}\right)=\left[\begin{array}{cc}
U_{+}^{\epsilon=\pi}\left(k_{y}\right) & 0 \\
0 & U_{-}^{\epsilon=\pi}\left(k_{y}\right)
\end{array}\right] .
$$

In our system, $N_{\text {occ }}=2$ and thus $U_{+}^{\epsilon=0, \pi}\left(k_{y}\right)$ are complex numbers, we can define a winding number at $\epsilon=0, \pi$ as

$$
W_{v_{x}}^{\epsilon}=\frac{1}{2 \pi i} \int_{0}^{2 \pi} d k_{y} \partial_{k_{y}} \log \left[U_{+}^{\epsilon}\left(k_{y}\right)\right],
$$

\section{Gauge transformation}

Since the Wilson line is determined by the occupied eigenstates of a Hamiltonian, the winding number of the Wilson line introduced in the preceding section may be dependent of the gauge transformation of the occupied eigenstates. Specifically, if we multiply a global phase to an occupied eigenstate, i.e., $\left|u_{k_{x}^{*}, k_{y}}^{m_{x}}\right\rangle \rightarrow e^{i \theta_{m_{x}}\left(k_{x}^{*}, k_{y}\right)}\left|u_{k_{x}^{*}, k_{y}}^{m_{x}}\right\rangle$ with $k_{x}^{*}=0, \pi$ and a reflection eigenvalue $m_{x}= \pm 1$, then

$$
\mathcal{W}_{\pi \leftarrow 0}^{\epsilon}\left(k_{y}\right) \rightarrow L^{\dagger}\left(k_{x}=\pi, k_{y}\right) \mathcal{W}_{\pi \leftarrow 0}^{\epsilon}\left(k_{y}\right) L\left(k_{x}=0, k_{y}\right),
$$

where

$$
L\left(k_{x}, k_{y}\right)=\left[\begin{array}{cc}
e^{i \theta_{+1}\left(k_{x}, k_{y}\right)} & 0 \\
0 & e^{i \theta_{-1}\left(k_{x}, k_{y}\right)}
\end{array}\right] .
$$

This gives us the transformation for the Wilson line for $\epsilon=$
Thus, the winding number changes to

$$
\begin{aligned}
W_{v_{x}}^{\epsilon} & \rightarrow \frac{1}{2 \pi i} \int_{0}^{2 \pi} d k_{y} \partial_{k_{y}} \log \left[U_{+}^{\epsilon}\left(k_{y}\right) e^{i \Theta^{\epsilon}}\left(k_{y}\right)\right], \\
& =N_{v_{x}}^{\epsilon}+\frac{1}{2 \pi}\left[\Theta^{\epsilon}\left(k_{y}=2 \pi\right)-\Theta^{\epsilon}\left(k_{y}=0\right)\right],
\end{aligned}
$$

where $\Theta^{\epsilon=0} \equiv \theta_{-1}\left(0, k_{y}\right)-\theta_{+1}\left(\pi, k_{y}\right), \Theta^{\epsilon=\pi} \equiv \theta_{+1}\left(0, k_{y}\right)-$ $\theta_{+1}\left(\pi, k_{y}\right)$. This shows that the global phase at $k_{x}=0, \pi$ can produce an unphysical change of the winding number once the global phase exhibits a winding, implying that an isolated value of the winding number is not physical. However, if we maintain this global phase unchanged as we vary a system parameter and observe the change of the winding number, then this change is physical and tells us that the edge polarization appears or disappears. This works as the polarization is a $Z_{2}$ quantity.

Numerically, we need to maintain the continuity of the global phase of the occupied energy states with respect to $k_{y}$ along the reflection symmetric lines $k_{x}=0$ and $k_{x}=\pi$ for a fixed system parameter $\gamma$ and to maintain the continuity of the Berry phase of these states about $k_{y}$ with respect to $\gamma$.

To maintain the continuity of the global phase with respect to $k_{y}$, we first make the wave function $\left|u_{k_{x}^{*}, k_{y}}^{m_{x}}\right\rangle$ continuous between two neighboring points $\left(k_{x}^{*}, k_{y}\right)$ and $\left(k_{x}^{*}, k_{y}+\Delta k_{y}\right)$, where $k_{y}=n \Delta k_{y}$ with $\Delta k_{y}=\frac{2 \pi}{N_{y}}$ and $n=0,1, \ldots,\left(N_{y}-1\right)$. To achieve this, we calculate the overlap between the two wave functions of neighboring momenta $\left\langle u_{k_{x}^{*}, k_{y}+\Delta k_{y}}^{m_{x}} \mid u_{k_{x}^{*}, k_{y}}^{m_{x}}\right\rangle$, and eliminate the numerical phase difference by the transformation,

$$
\left|u_{k_{x}^{*}, k_{y}+\Delta k_{y}}^{m_{x}}\right\rangle \rightarrow e^{i \phi_{1}}\left|u_{k_{x}^{*}, k_{y}+\Delta k_{y}}^{m_{x}}\right\rangle,
$$

where $\phi_{1}=\operatorname{Im} \log \left\langle u_{k_{x}^{*}, k_{y}+\Delta k_{y}}^{m_{x}} \mid u_{k_{x}^{*}, k_{y}}^{m_{x}}\right\rangle$. We repeat this process from $k_{y}=0$ to $k_{y}=2 \pi-\Delta k_{y}$. After that, we make the wave functions continuous between $k_{y}=2 \pi-\Delta k_{y}$ and $k_{y}=0$ by performing the following phase transformations

$$
\left|u_{k_{x}^{*}, n \Delta k_{y}}^{m_{x}}\right\rangle \rightarrow e^{-i \phi_{2} n / N_{y}}\left|u_{k_{x}^{*}, n \Delta k_{y}}^{m_{x}}\right\rangle,
$$

where $\phi_{2}=\operatorname{Im} \log \left\langle u_{k_{x}^{*}, 0}^{m_{x}} \mid u_{k_{x}^{*}, 2 \pi-\Delta k_{y}}^{m_{x}}\right\rangle$. 

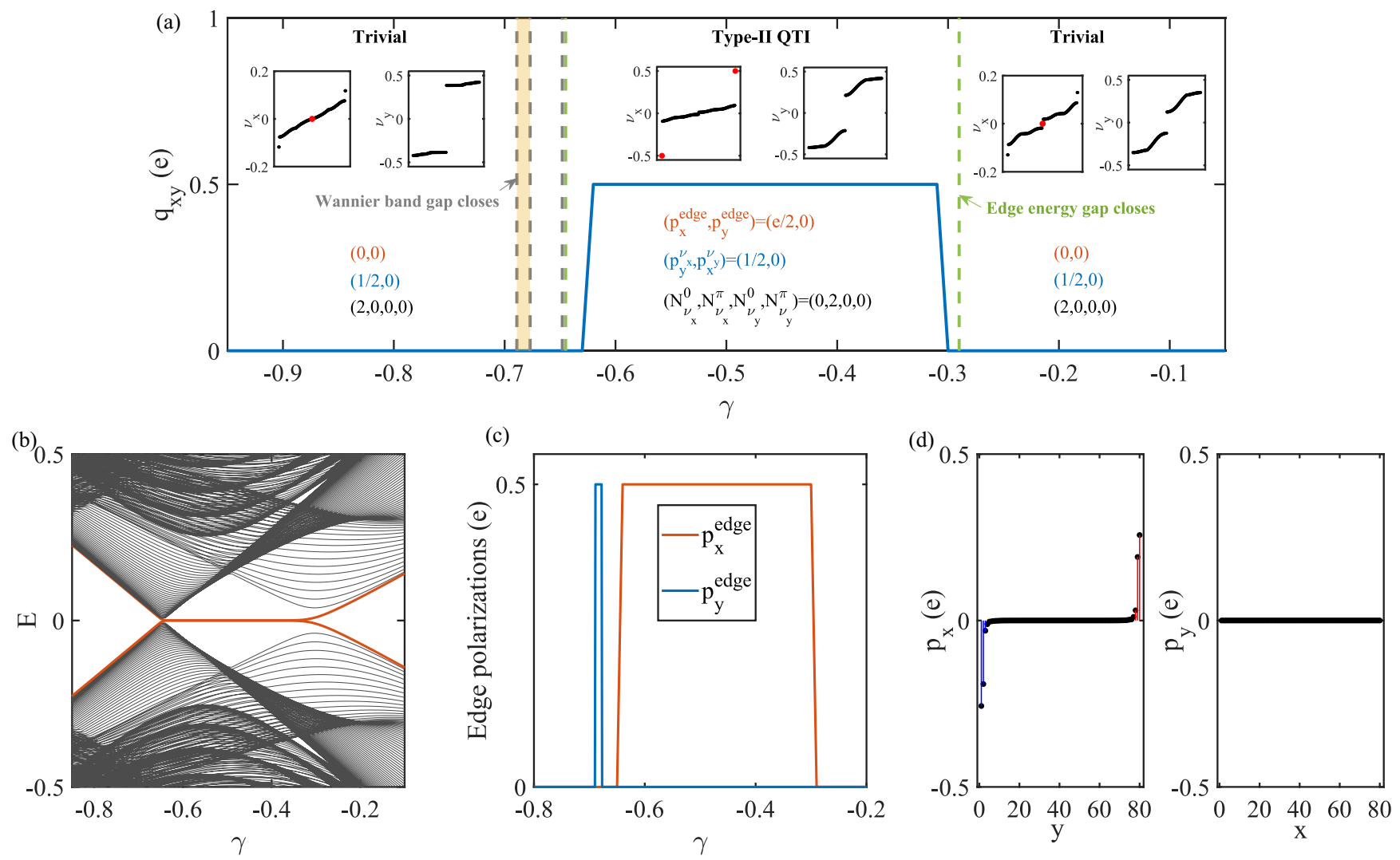

FIG. 13. (a) Phase diagram for the Hamiltonian $H_{I I}$ (with $b_{2}=1.2$ and $g_{0}=-0.29$ ) versus $\gamma$, where the quadrupole moment is plotted as a blue line. It displays the topologically trivial insulator, the type-II QTI and the new phase with nonzero edge polarizations but without quadrupole moments and zero-energy corner modes (displayed in the light red region). The subsets display the same quantities as in Fig. 2. The small discrepancy between the edge gap closing points and the quadrupole moment transition points is caused by the finite-size effects for calculating the quadrupole moment in a finite-size system with $N=240$. (b) The energy spectrum with respect to $\gamma$ for open boundary conditions along all directions with zero-energy corner modes being highlighted by a red line. (c) The edge polarizations calculated based on the formula (15) by choosing a gauge such that $W_{v_{x}}^{\epsilon=\pi}\left(\gamma_{0}=-0.8\right)=W_{v_{y}}^{\epsilon=\pi}\left(\gamma_{0}=-0.8\right)=0$ because it is topologically trivial when $\gamma=-0.8$. (d) The spatial distribution of the edge polarization in a type-II QTI with $\gamma=-0.4$.

To make sure that the Berry phase of each occupied band about $k_{y}$ along the reflection symmetric lines $k_{x}^{*}=0, \pi$ is continuous as $\gamma$ varies, we numerically compute the Berry phase based on the following formula:

$$
v_{y}^{ \pm 1}\left(k_{x}^{*}=0, \pi\right)=\operatorname{Im} \lim _{N_{y} \rightarrow \infty} \sum_{n=0}^{N_{y}-1} \log \left\langle u_{k_{x}^{*},(n+1) \Delta k_{y}}^{ \pm 1} \mid u_{k_{x}^{*}, n \Delta k_{y}}^{ \pm 1}\right\rangle
$$

and perform a suitable transformation $\left|u_{k_{x}^{*}, k_{y}=n \Delta k_{y}}^{m_{x}}\right\rangle \rightarrow$ $\exp \left(-i 2 \pi p \frac{n}{N_{y}}\right)\left|u_{k_{x}^{*}, k_{y}=n \Delta k_{y}}^{m_{x}}\right\rangle$ with $p$ being an integer provided that the Berry phases between two neighboring $\gamma$ change by $-2 p \pi$. The procedure is similar for calculation of the winding number $W_{v_{y}}^{\epsilon}$.

\section{APPENDIX E: MORE PHASE DIAGRAMS FOR SIMPLIFIED MODELS}

In the main text, we have demonstrated the existence of these new topological phenomena by studying systems with the particle-hole symmetry. In this Appendix, we will present phase diagrams for more models, in particular, the models with the chiral symmetry.
We first show the phase diagram and topological properties for the Hamiltonian $H_{I I}$ in Eq. (20) with $b_{2}=1.2$ and $g_{0}=$ -0.29 in Fig. 13. Similarly to Fig. 5, we can clearly see the existence of the type-II QTI in the phase diagram. This phase is also a normal type-II QTI given that the Wannier spectrum $v_{x}$ has edge states only at $v_{x}= \pm 1 / 2$. Besides this type-II phase, we also observe the new topological phase with only nonzero $p_{y}^{\text {edge }}$ in the light red region, which arises due to the Wannier gap closing at $v_{y}= \pm 1 / 2$. We note that across $\gamma=-0.645$, the edge polarization $p_{x}^{\text {edge }}$ appears due to the the $y$-normal edge energy gap closing rather than the Wannier gap closing. The Wannier gap closing happens at $v_{x}=0$ and thus does not contribute to the edge polarizations.

Similarly, we map out the phase diagram for the Hamiltonian $H_{I I I}$ in Eq. (23) with $b_{2}=1.2$ and $g_{0}=0.65$ [see Fig. 14]. This Hamiltonian preserves both the particle-hole and chiral symmetry. Evidently, the phase diagram exhibits the type-II QTI as well as the new topological phase with only quantized edge polarizations. By adding a term $\alpha \sin k_{x} \sigma_{1} \otimes$ $\sigma_{1}$ that breaks the particle-hole symmetry but preserves the chiral symmetry, we can still observe these topological phenomena, indicating that these phases can arise for a system with the chiral symmetry. 

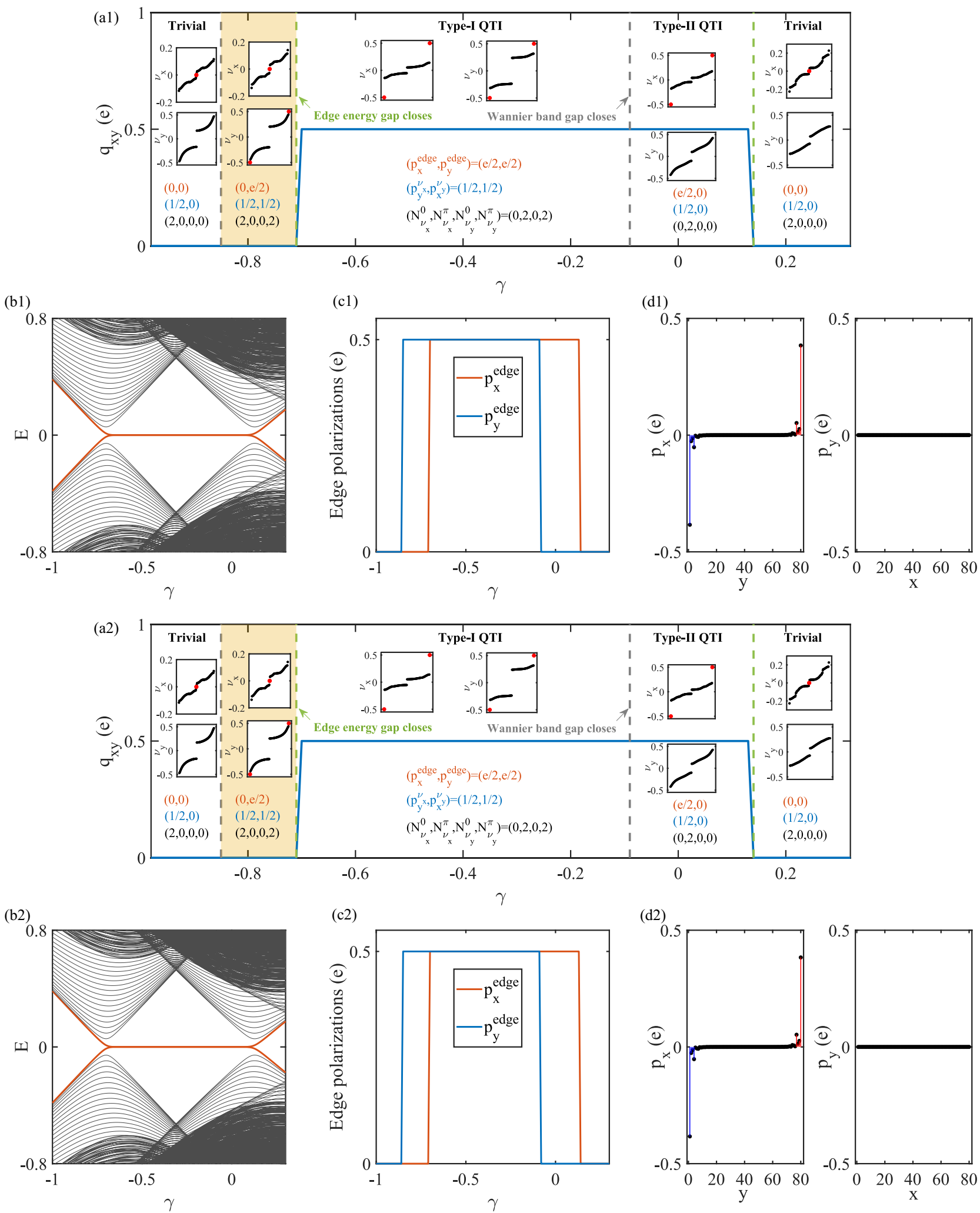

FIG. 14. (a1) Phase diagram for the Hamiltonian $H_{I I I}$ (with $b_{2}=1.2$ and $g_{0}=0.65$ ) versus $\gamma$, where the quadrupole moment is plotted as a blue line. The light red region shows the new topological phase with nonzero edge polarizations but without quadrupole moments and zero-energy corner modes. The subsets display the same quantities as in Fig. 2. (b1) The energy spectrum with respect to $\gamma$ for open boundary conditions along all directions with zero-energy corner modes being denoted by a red line. (c1) The edge polarizations calculated based on the formula (15) by choosing a gauge such that $W_{v_{x}}^{\epsilon=\pi}\left(\gamma_{0}=-1\right)=W_{v_{y}}^{\epsilon=\pi}\left(\gamma_{0}=-1\right)=0$ because it is topologically trivial when $\gamma=-1$. (d1) The spatial profiles of the edge polarization in a type-II QTI. Panels (a2)-(d2) display the same quantities as (a1)-(d1) but for the Hamiltonian $H_{I I I}+\alpha \sin k_{x} \sigma_{1} \otimes \sigma_{1}$ (with $b_{2}=1.2, g_{0}=0.65$, and $\alpha=0.2$ ). We use $N=80$ for evaluation of the quadrupole moment. 

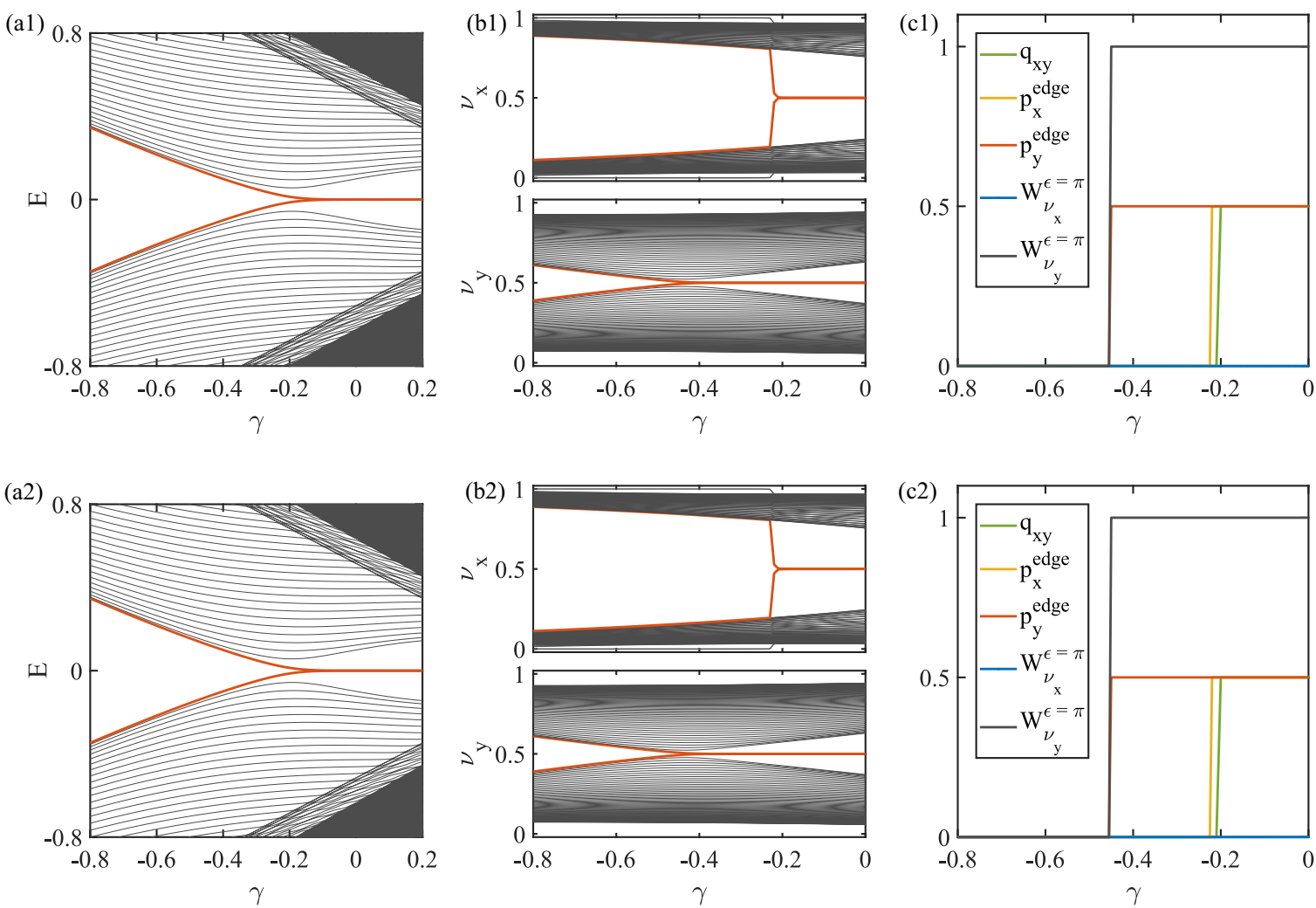

FIG. 15. [(a1) and (a2)] The energy spectrum versus $\gamma$ in a geometry with open boundaries along all directions. [(b1) and (b2)] The Wannier band $v_{x}$ and $v_{y}$ versus $\gamma$. [(c1) and (c2)] The quadrupole moment $q_{x y}$, the edge polarizations $p_{\mu}^{\text {edge }}(\mu=x, y)$ computed using the formula (15) and the winding numbers $W_{v_{\mu}}^{\epsilon=\pi}(\mu=x, y)$ computed using the formula (13) versus $\gamma$. When calculating the edge polarizations and the winding number, we choose a gauge such that $W_{v_{x}}^{\epsilon=\pi}\left(\gamma_{0}=-0.8\right)=W_{v_{y}}^{\epsilon=\pi}\left(\gamma_{0}=-0.8\right)=0$ because the phase is topologically trivial when $\gamma=-0.8$. $q_{x y}$ and $p_{x}^{\text {edge }}$ are hidden behind the blue line when $\gamma<-0.22$. The small discrepancy between the quadrupole moment transition point and the edge gap closing point is caused by the finite-size effects. Here we use $N=80$ for evaluation of the quadrupole moment. In (a1)-(c1) and (a2)-(c2), we consider the Hamiltonian $H_{I V}+\alpha \sin k_{x} \sigma_{1} \otimes \sigma_{1}$ with $b_{2}=1.2$ and $\alpha=0.2$, and the Hamiltonian $H_{I V}+\alpha \sin k_{x} \sigma_{3} \otimes \sigma_{3}$ with $b_{2}=1.2$ and $\alpha=0.2$, respectively.

Finally, in Fig. 15, we provide the energy spectra, the Wannier spectrum, the quadrupole moment, the edge polarizations and the winding number for the Hamiltonian $\mathrm{H}_{I V}+$ $\alpha \sin k_{x} \sigma_{1} \otimes \sigma_{1}$ with $b_{2}=1.2$ and $\alpha=0.2$ that preserves the chiral symmetry and $H_{I V}+\alpha \sin k_{x} \sigma_{3} \otimes \sigma_{3}$ with $b_{2}=1.2$ and $\alpha=0.2$ that preserves the particle-hole symmetry. We can see that all these figures are very similar to Fig. 6 in the main text, further reflecting that the new topological phase can appear in a system with either the particle-hole symmetry or the chiral symmetry.

\section{APPENDIX F: ENERGY AND WANNIER SPECTRA DURING A PUMPING PROCESS}

In the main text, we have shown the transport of the corner charge, the quadrupole moment and the edge polarizations as system parameters vary over an entire cycle. In this Appendix, we present the energy spectrum in an open boundary geometry (see the first column in Fig. 16) and the Wannier spectrum in a cylinder geometry (see the last two columns in Fig. 16) during this full process. The first row in the figure corresponds to the pump from a topologically trivial phase to a type-II AQTI and then back to the trivial phase, while the second row to the pump from a type-II AQTI to a type-I AQTI and then back to the original type-II phase. It is clear that we can divide the energy spectrum and Wannier spectrum into two bands with gaps between them. In the former scenario, we see that the corner states connect the lower energy band to the higher one as time evolves, which shows the chiral property of the corner states if time is regarded as a third momentum; this agrees well with the quantized transport of corner charges shown in the main text. For the Wannier spectrum $v_{x}$, the edge states exist inside both the gaps around 0 and $1 / 2$ and these states connect two bands of the Wannier spectrum as time progresses, similarly to the energy spectrum. However, for the Wannier spectrum $v_{y}$, we do not see the presence of the edge states connecting two Wannier bands, consistent with the zero net transport for the edge polarization $p_{y}^{\text {edge }}$. In the second pumping scenario, while the corner states exist during the full cycle, they do not connect the two energy bands, and thus are not "chiral," which is consistent with the zero transport of the corner charges. Similar band patterns occur in the Wannier spectrum $v_{x}$. However, for the Wannier spectrum $v_{y}$, we find the "chiral"-like edge states, which explains the presence of the net transport of the edge polarization $p_{y}^{\text {edge }}$ shown in the main text. 
(a)

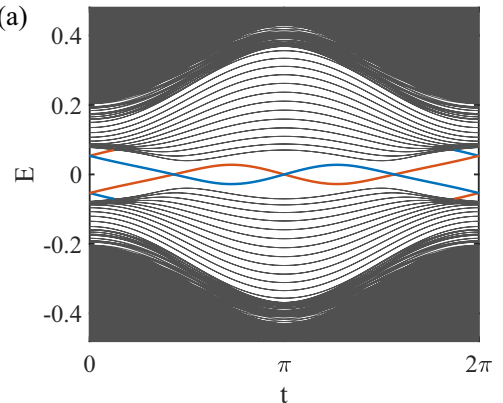

(b)

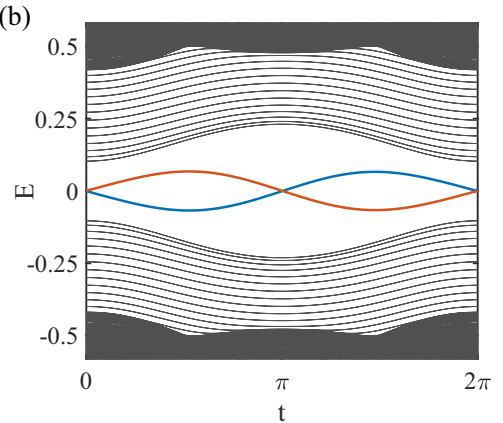

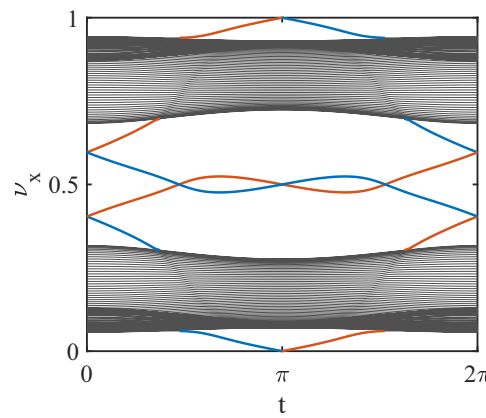
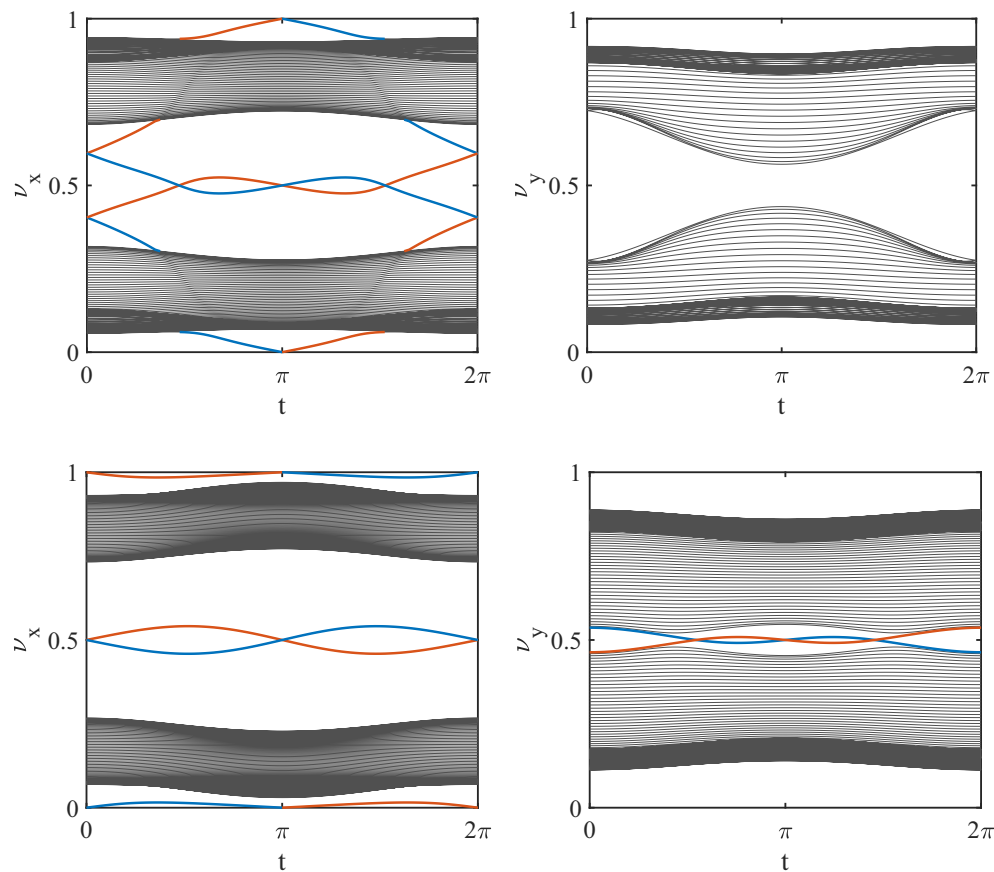

FIG. 16. The energy spectrum and Wannier spectrum during the pumping process. (a) The cycle from a topologically trivial phase to a type-II AQTI and back to the trivial phase. (b) The cycle from a type-II AQTI to a type-I AQTI and back to the type-II AQTI. For both (a) and (b) the first column corresponds to the energy spectrum with open boundary along $x$ and $y$ and the second (third) column correspond to the Wannier spectrum $v_{x}\left(v_{y}\right)$ with open boundary along $y(x)$ and periodic boundary along $x(y)$.

\section{APPENDIX G: ENTANGLEMENT SPECTRA}

In this Appendix, we will define the entanglement spectra and show how to calculate them. Let us first partition a system into two subsystems labeled by $A$ and $B$, respectively. The reduced density matrix for the subsystem $A$ can be obtained by performing partial trace over the subsystem $B$, that is,

$$
\rho_{A}=\operatorname{Tr}_{B}|\Psi\rangle\langle\Psi|=\frac{e^{-H_{A}}}{Z_{A}},
$$

where $|\Psi\rangle$ is a many-body ground state, $H_{A}$ is defined as a Hamiltonian corresponding to the reduced density matrix $\rho_{A}$ and $Z_{A}=\operatorname{Tr} e^{-H_{A}}$. The entanglement spectrum refers to the eigenvalues of $\rho_{A}[70,80,81]$.

In the single-particle case, the entanglement spectrum can be determined by diagonalizing the correlation matrix [82]

$$
\left[C_{A}\right]_{i j}=\left\langle\hat{c}_{i}^{\dagger} \hat{c}_{j}\right\rangle,
$$

where $i, j \in A$. If we diagonalize $H_{A}$ as $H_{A}=\sum_{n} \varepsilon_{n} \hat{a}_{n}^{\dagger} \hat{a}_{n}$, then the single-particle entanglement spectrum $\xi_{n}$ and $\varepsilon_{n}$ are related by

$$
\xi_{n}=\frac{1}{e^{\varepsilon_{n}}+1} .
$$

Clearly, the entanglement spectrum $\xi_{n}=0.5$ corresponds to an entanglement zero mode $\varepsilon_{n}=0$. In the main text, we show the entanglement spectrum $\mathrm{ES}_{x}$ and $\mathrm{ES}_{y}$ obtained by tracing out the right part and top part of a system as shown in Figs. 17(a) and 17(b), respectively.

To characterize the edge modes of quadrupole topological insulators, which are localized at the corners, nested entanglement spectra are introduced [8], as detailed in the following.
For a 2D quadrupole insulator, the Hamiltonian can be diagonalized as

$$
H=\sum_{k_{x}, k_{y}, n} E_{k_{x}, k_{y}}^{n} \hat{f}_{k_{x}, k_{y}, n}^{\dagger} \hat{f}_{k_{x}, k_{y}, n}
$$

where $\hat{f}_{k_{x}, k_{y}, n}^{\dagger}=\sum_{\alpha}\left[u_{k_{x}, k_{y}}^{n}\right]^{\alpha} \quad \hat{c}_{k_{x}, k_{y}, \alpha}^{\dagger}$ and $\left|u_{k_{x}, k_{y}}^{n}\right\rangle$ is the $n$th eigenstate of $H(\mathbf{k})$ corresponding to the eigenenergy $E_{k_{x}}^{n}, k_{y}$. Suppose that the system has $L_{x} \times L_{y}$ unit cells with $L_{x}=$ $2 N_{x}$ and $L_{y}=2 N_{y}$. We first partition the system into two subsystems $A$ and $B$ along the $x$ direction: $A=\{(x, y) \mid 1 \leqslant$ $\left.x \leqslant N_{x}, 1 \leqslant y \leqslant L_{y}\right\}$ and $B=\left\{(x, y) \mid N_{x}<x \leqslant L_{x}, 1 \leqslant y \leqslant\right.$ $\left.L_{y}\right\}$. The correlation matrix in the subsystem $A$ is given by

$$
\begin{aligned}
{\left[C_{A, k_{y}}\right]_{x \alpha, x^{\prime} \alpha^{\prime}} } & =\left\langle\Psi_{G}\left|\hat{c}_{x \alpha, k_{y}}^{\dagger} \hat{c}_{x^{\prime} \alpha^{\prime}, k_{y}}\right| \Psi_{G}\right\rangle \\
& =\frac{1}{L_{x}} \sum_{k_{x}} e^{i k_{x}\left(x-x^{\prime}\right)} \sum_{n \in o c c}\left[U_{k_{x}, k_{y}}\right]_{\alpha n}\left[U_{k_{x}, k_{y}}^{\dagger}\right]_{n \alpha^{\prime}},
\end{aligned}
$$

(a)

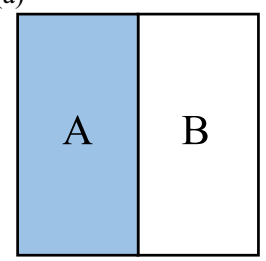

(b)

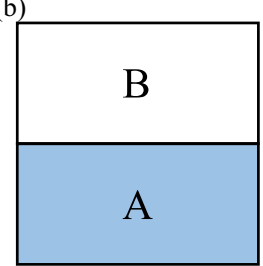

(c)

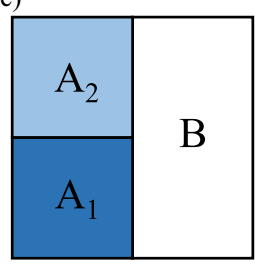

FIG. 17. The entanglement spectrum $\mathrm{ES}_{x}$ in (a) and $\mathrm{ES}_{y}$ in (b) are evaluated by partitioning a system into two subsystems $A$ and $B$ and tracing out the subsystem $B$. (c) The nested entanglement spectrum $\mathrm{ES}_{x y}$ is obtained by further tracing out the subsystem $A_{2}$. 
where $\left|\Psi_{G}\right\rangle$ is the many-body ground state of $H$ and $U_{k_{x}, k_{y}}$ consists of all occupied eigenstates $\left|u_{k_{x}, k_{y}}^{n}\right\rangle$ as column vectors. Diagonalizing $C_{A, k_{y}}$ yields the eigenvalues $\xi_{k_{y}}^{m}$ and eigenvectors $\left|v_{k_{y}}^{m}\right\rangle$ of $C_{A, k_{y}}$. The Hamiltonian $H_{A}$ of the reduced density matrix $\rho_{A}$ can be diagonalized as

$$
H_{A}=\sum_{k_{y}, m} \log \left(\frac{1}{\xi_{k_{y}}^{m}}-1\right) \hat{g}_{k_{y}, m}^{\dagger} \hat{g}_{k_{y}, m},
$$

where $g_{k_{y}, m}^{\dagger}=\sum_{\alpha x}\left[v_{k_{y}}^{m}\right]_{x \alpha} c_{x \alpha, k_{y}}^{\dagger}$. The subsystem $A$ is further partitioned into two parts along the $y$ direction [as shown in Fig. 17(c)]: $A_{1}=\left\{(x, y) \mid 1 \leqslant x \leqslant N_{x}, 1 \leqslant y \leqslant N_{y}\right\}$ and $A_{2}=$ $\left\{(x, y) \mid 1 \leqslant x \leqslant N_{x}, N_{y}<y \leqslant L_{y}\right\}$. The correlation matrix in the region $A_{1}$ is given by

$$
\begin{aligned}
{\left[C_{A_{1}}\right]_{x y \alpha, x^{\prime} y^{\prime} \alpha^{\prime}} } & =\left\langle\Psi_{A}\left|c_{x y \alpha}^{\dagger} c_{x^{\prime} y^{\prime} \alpha^{\prime}}\right| \Psi_{A}\right\rangle \\
& =\frac{1}{L_{y}} \sum_{k_{y}} e^{i k_{y}\left(y-y^{\prime}\right)} \sum_{m \in o c c}\left[V_{k_{y}}\right]_{x \alpha, m}\left[V_{k_{y}}^{\dagger}\right]_{m, x^{\prime} \alpha^{\prime}},
\end{aligned}
$$

where $\left|\Psi_{A}\right\rangle$ is the many-body ground state of $H_{A}$ and the average is over all occupied states of $H_{A} . V_{k_{y}}$ is made up of all occupied eigenvectors $\left|v_{k_{y}}^{m}\right\rangle$ as column vectors. We can determine the nested entanglement spectrum by calculating the eigenvalues of $C_{A_{1}}$.

\section{APPENDIX H: EXPERIMENTAL REALIZATION}

In this Appendix, we discuss how to realize our Hamiltonian in electric circuits, in which the SSH model [77], Weyl semimetal [83], and type-I quadrupole topological insulator [26] have been experimentally achieved. Let us consider an electrical network consisting of many nodes simulating sites in a tight-binding model. For each node $m$ in the circuit, suppose that $I_{m}$ is the external current flowing into this node and $V_{m}$ is the voltage at this node with respect to the ground, according to Kirchhoff's law, we have

$$
I_{m}=\sum_{n} I_{m n}+I_{m 0}=\sum_{n} X_{m n}\left(V_{m}-V_{n}\right)+X_{m} V_{m},
$$

where $I_{m n}$ and $I_{m 0}$ are the current flowing from node $m$ to $n$ and from node $m$ to the ground, respectively. $X_{m n}=1 / Z_{m n}$ is the admittance between node $m$ and $n$ with $Z_{m n}$ the corresponding impedance, and $X_{m}=1 / Z_{m}$ is the admittance between node $m$ and the ground. Writing $I_{m}$ and $V_{m}$ in the form of column vectors, we have

$$
\mathbf{I}=J(\omega) \mathbf{V},
$$

where $J(\omega)$ denotes the circuit Laplacian with $\omega$ being the AC frequency of the input current.

By connecting appropriate capacitors, inductors and negative impedance converters with current inversion (INIC) [78,79] as shown in Fig. 10 in the main text, we can achieve a Laplacian simulating our Hamiltonian, i.e., $J=i H$. The sign of the resistance of a INIC depends on how it is connected. For example, for the configuration shown in Fig. 10 in the main text, the current from node 1 to node 2 within a unit cell is determined by $I_{12}=\left(V_{1}-V_{2}\right) /(-R)$ corresponding to a negative resistance while the current from node 2 to node 1 determined by $I_{21}=\left(V_{2}-V_{1}\right) / R$ corresponding to a positive resistance. To eliminate unnecessary onsite terms, we also need to add onsite impedances $Z_{m}^{\prime}$ as shown in Fig. 10 in the main text. For the system with periodic boundary conditions, the values of $Z_{a=1,2,3,4}^{\prime}$ are as follows:

$$
\begin{aligned}
\frac{1}{Z_{1}^{\prime}}= & \frac{1}{Z_{4}^{\prime}}=\left(2 t_{1}^{\prime}-2 t_{2}-2 t_{2}^{\prime}+\Delta\right) \\
& +i\left(4 t_{1}+2 t_{1}^{\prime}+6 t_{2}-2 t_{2}^{\prime}\right), \\
\frac{1}{Z_{2}^{\prime}}= & \frac{1}{Z_{3}^{\prime}}=\left(-2 t_{1}^{\prime}+2 t_{2}+2 t_{2}^{\prime}-\Delta\right) \\
& +i\left(2 t_{1}^{\prime}+2 t_{2}-6 t_{2}^{\prime}+2 \gamma\right) .
\end{aligned}
$$

We follow the experimental approach to directly measure the Green's function [84]. Specifically, we apply an input current $I_{n}$ at one node $n$ of the circuit and measure the voltage $V_{m}^{(n)}$ at node $m$, giving us the single-point impedance,

$$
G_{m n}=V_{m}^{(n)} / I_{n}
$$

which is a Green's function of the system Hamiltonian, i.e.,

$$
G_{m n}=\left(J^{-1}\right)_{m n} \text {. }
$$

All the information, such as the energy spectrum and eigenstates, can be extracted from the Green's function.

To achieve our model, we consider an electric circuit composed of $N_{x} N_{y}$ unit cells; each unit cell contains four nodes and each node is labeled by $(\mathbf{R}, \alpha)$. If we consider a torus geometry with periodic boundaries along both $x$ and $y$, we only need to apply a current in one node $(\mathbf{0}, \beta)$ and measure the impedance $G_{\alpha \beta}(\mathbf{R})$ between the node $(\mathbf{R}, \alpha)$ and node $(\mathbf{0}, \beta)$ for all the different nodes $(\mathbf{R}, \alpha)$, leading to the Green's function in momentum space,

$$
G_{\alpha \beta}(\mathbf{k})=\sum_{\mathbf{R}} G_{\alpha \beta}(\mathbf{R}) \exp (-i \mathbf{k} \cdot \mathbf{R}),
$$

where $\mathbf{k}$ is the momentum and $\sum_{\mathbf{R}}$ is the sum over all unit cells. Similarly, in a cylinder geometry, e.g., with open boundaries along $y$ and periodic boundaries along $x$, only the impedance $Z_{\alpha \beta}\left(\left(R_{x}, R_{y}\right),\left(0, R_{y}^{\prime}\right)\right)=$ $G_{\alpha \beta}\left(\left(R_{x}, R_{y}\right),\left(0, R_{y}^{\prime}\right)\right)$ between the node $\left(\left(R_{x}, R_{y}\right), \alpha\right)$ and node $\left(\left(0, R_{y}^{\prime}\right), \beta\right)$ is required to be probed, yielding the Green's function

$$
G_{\left(R_{y}, \alpha\right)\left(R_{y}^{\prime}, \beta\right)}(\mathbf{k})=\sum_{R_{x}} G_{\alpha \beta}\left(\left(R_{x}, R_{y}\right),\left(0, R_{y}^{\prime}\right)\right) \exp \left(-i k_{x} R_{x}\right) .
$$

Once the Green's functions in the torus and cylinder geometry are measured, the quadrupole moment and edge polarizations can be obtained.

For the system with open boundary conditions, the presence of zero-energy corner states of the Hamiltonian will give rise to the divergence of the two-point impedance near the corners. Thus we can measure the corner modes through the measurement of the resonance of the impedance between two neighboring nodes near the corners at the resonance frequency [26]. 
[1] W. A. Benalcazar, B. A. Bernevig, and T. L. Hughes, Quantized electric multipole insulators, Science 357, 61 (2017).

[2] W. A. Benalcazar, B. A. Bernevig, and T. L. Hughes, Electric multipole moments, topological multipole moment pumping, and chiral hinge states in crystalline insulators, Phys. Rev. B 96, 245115 (2017).

[3] M. Sitte, A. Rosch, E. Altman, and L. Fritz, Topological Insulators in Magnetic Fields: Quantum Hall Effect and Edge Channels with a Nonquantized $\theta$ Term, Phys. Rev. Lett. 108, 126807 (2012).

[4] F. Zhang, C. L. Kane, and E. J. Mele, Surface State Magnetization and Chiral Edge States on Topological Insulators, Phys. Rev. Lett. 110, 046404 (2013).

[5] R.-J. Slager, L. Rademaker, J. Zaanen, and L. Balents, Impurity-bound states and Green's function zeros as local signatures of topology, Phys. Rev. B 92, 085126 (2015).

[6] Z. Song, Z. Fang, and C. Fang, $(d-2)$-Dimensional Edge States of Rotation Symmetry Protected Topological States, Phys. Rev. Lett. 119, 246402 (2017).

[7] J. Langbehn, Y. Peng, L. Trifunovic, F. von Oppen, and P. W. Brouwer, Reflection-Symmetric Second-Order Topological Insulators and Superconductors, Phys. Rev. Lett. 119, 246401 (2017).

[8] F. Schindler, A. M. Cook, M. G. Vergniory, Z. Wang, S. S. P. Parkin, B. A. Bernevig, and T. Neupert, Higher-order topological insulators, Sci. Adv. 4, eaat0346 (2018).

[9] F. Schindler, Z. Wang, M. G. Vergniory, A. M. Cook, A. Murani, S. Sengupta, A. Y. Kasumov, R. Deblock, S. Jeon, I. Drozdov, H. Bouchiat, S. Guéron, A. Yazdani, B. A. Bernevig, and T. Neupert, Higher-order topology in bismuth, Nat. Phys. 14, 918 (2018).

[10] Y. Xu, R. Xue, and S. Wan, Topological corner states on kagome lattice based chiral higher-order topological insulator, arXiv:1711.09202 (2017).

[11] H. Shapourian, Y. Wang, and S. Ryu, Topological crystalline superconductivity and second-order topological superconductivity in nodal-loop materials, Phys. Rev. B 97, 094508 (2018).

[12] M. Lin and T. L. Hughes, Topological quadrupolar semimetals, Phys. Rev. B 98, 241103(R) (2018).

[13] M. Ezawa, Higher-Order Topological Insulators and Semimetals on the Breathing Kagome and Pyrochlore Lattices, Phys. Rev. Lett. 120, 026801 (2018).

[14] E. Khalaf, Higher-order topological insulators and superconductors protected by inversion symmetry, Phys. Rev. B 97, 205136 (2018).

[15] M. Geier, L. Trifunovic, M. Hoskam, and P. W. Brouwer, Second-order topological insulators and superconductors with an order-two crystalline symmetry, Phys. Rev. B, 97, 205135 (2018).

[16] S. Franca, J. van den Brink, and I. C. Fulga, An anomalous higher-order topological insulator, Phys. Rev. B 98, 201114(R) (2018).

[17] Z. Yan, F. Song, and Z. Wang, Majorana Corner Modes in a High-Temperature Platform, Phys. Rev. Lett. 121, 096803 (2018).

[18] Q. Wang, C.-C. Liu, Y.-M. Lu, and F. Zhang, High-Temperature Majorana Corner States, Phys. Rev. Lett. 121, 186801 (2018).
[19] D. Călugăru, V. Juričić, and B. Roy, Higher-order topological phases: A general principle of construction, Phys. Rev. B 99. 041301(R) (2019).

[20] L. Trifunovic and P. W. Brouwer, Higher-Order Bulk-Boundary Correspondence for Topological Crystalline Phases, Phys. Rev. X 9, 011012 (2019).

[21] F. Liu, H.-Y. Deng, and K. Wakabayashi, Helical Topological Edge States in a Quadrupole Phase, Phys. Rev. Lett. 122, 086804 (2019).

[22] X.-L. Sheng, C. Chen, H. Liu, Z. Chen, Z.-M. Yu, Y. X. Zhao and S. A. Yang, Two-Dimensional Second-Order Topological Insulator in Graphdiyne, Phys. Rev. Lett. 123, 256402 (2019).

[23] H. Li and K. Sun, Pfaffian Formalism for Higher-Order Topological Insulators, Phys. Rev. Lett. 124, 036401 (2020).

[24] M. Serra-Garcia, V. Peri, R. Süsstrunk, O. R. Bilal, T. Larsen, L. G. Villanueva, and S. D. Huber, Observation of a phononic quadrupole topological insulator, Nature 555, 342 (2018).

[25] C. W. Peterson, W. A. Benalcazar, T. L. Hughes, and G. Bahl, A quantized microwave quadrupole insulator with topologically protected corner states, Nature 555, 346 (2018).

[26] S. Imhof, C. Berger, F. Bayer, J. Brehm, L. W. Molenkamp, T. Kiessling, F. Schindler, C. H. Lee, M. Greiter, T. Neupert, and R. Thomale, Topolectrical-circuit realization of topological corner modes, Nat. Phys. 14, 925 (2018).

[27] E. Khalaf, W. A. Benalcazar, T. L. Hughes, and R. Queiroz, Boundary-obstructed topological phases, arXiv:1908.00011 (2019).

[28] L. Fidkowski, T. S. Jackson, and I. Klich, Model Characterization of Gapless Edge Modes of Topological Insulators using Intermediate Brillouin-Zone Functions, Phys. Rev. Lett. 107, 036601 (2011).

[29] M. S. Rudner, N. H. Lindner, E. Berg, and M. Levin, Anomalous Edge States and the Bulk-Edge Correspondence for Periodically Driven Two-Dimensional Systems, Phys. Rev. X 3, 031005 (2013).

[30] D. Leykam, S. Mittal, M. Hafezi, and Y. D. Chong, Reconfigurable Topological Phases in Next-Nearest-Neighbor Coupled Resonator Lattices, Phys. Rev. Lett. 121, 023901 (2018).

[31] M. Li, D. Zhirihin, M. Gorlach, et al. Higher-order topological states in photonic kagome crystals with long-range interactions, Nat. Photon. 14, 89 (2020).

[32] S. V. Syzranov, M. L. Wall, V. Gurarie and A. M. Rey, Spin Corbital dynamics in a system of polar molecules, Nat. Commun. 5, 5391 (2014).

[33] D. Peter, N. Y. Yao, N. Lang, S. D. Huber, M. D. Lukin, and H. P. Büchler, Topological bands with a Chern number $\mathrm{C}=2$ by dipolar exchange interactions, Phys. Rev. A 91, 053617 (2015).

[34] A. Browaeys, D. Barredo, and T. Lahaye, Experimental investigations of dipole Cdipole interactions between a few Rydberg atoms, J. Phys. B 49, 152001 (2016).

[35] S. Weber, S. de Léséleuc, V. Lienhard, D. Barredo, T. Lahaye, A. Browaeys, and H. P. Büchler, Topologically protected edge states in small Rydberg systems, Quant. Sci. Technol. 3, 044001 (2018).

[36] S. de Léséleuc, V. Lienhard, P. Scholl, D. Barredo, S. Weber, N. Lang, H. P. Büchler, T. Lahaye, and A. Browaeys, Observation of a symmetry-protected topological phase of interacting bosons with Rydberg atoms, Science 365, 775 (2019). 
[37] G. C. Ménard et al., Coherent long-range magnetic bound states in a superconductor, Nat. Phys. 11, 1013 (2015).

[38] G. C. Ménard et al., Two-dimensional topological superconductivity in $\mathrm{Pb} / \mathrm{Co} / \mathrm{Si}(111)$, Nat. Commun. 8, 2040 (2017).

[39] J. Röntynen and T. Ojanen, Topological Superconductivity and High Chern Numbers in 2D Ferromagnetic Shiba Lattices, Phys. Rev. Lett. 114, 236803 (2015).

[40] B. W. Heinrich, J. I. Pascual, and K. J. Franke, Single magnetic adsorbates on s-wave superconductors, Prog. Surf. Sci. 93, 1 (2018).

[41] A. Eckardt, C. Weiss, and M. Holthaus, Superfluid-Insulator Transition in a Periodically Driven Optical Lattice, Phys. Rev. Lett. 95, 260404 (2005).

[42] M. DiLiberto, O. Tieleman, V. Branchina, and C. M. Smith, Finite-momentum Bose-Einstein condensates in shaken twodimensional square optical lattices, Phys. Rev. A 84, 013607 (2011).

[43] J. Struck, C. Ölschläger, M. Weinberg, P. Hauke, J. Simonet, A. Eckardt, M. Lewenstein, K. Sengstock, and P. Windpassinger, Tunable Gauge Potential for Neutral and Spinless Particles in Driven Optical Lattices, Phys. Rev. Lett. 108, 225304 (2012).

[44] L. D'Alessio and M. Rigol, Dynamical preparation of Floquet Chern insulators, Nat. Commun. 6, 8336 (2015).

[45] M. D. Caio, N. R. Cooper, and M. J. Bhaseen, Quantum Quenches in Chern Insulators, Phys. Rev. Lett. 115, 236403 (2015).

[46] S. Vajna and B. Dóra, Topological classification of dynamical phase transitions, Phys. Rev. B 91, 155127 (2015).

[47] J. C. Budich and M. Heyl, Dynamical topological order parameters far from equilibrium, Phys. Rev. B 93, 085416 (2016).

[48] Z. Huang and A. V. Balatsky, Dynamical Quantum Phase Transitions: Role of Topological Nodes in Wave Function Overlaps, Phys. Rev. Lett. 117, 086802 (2016).

[49] Y. Hu, P. Zoller, and J. C. Budich, Dynamical Buildup of a Quantized Hall Response from Nontopological States, Phys. Rev. Lett. 117, 126803 (2016).

[50] J. H. Wilson, J. C. W. Song, and G. Refael, Remnant Geometric Hall Response in a Quantum Quench, Phys. Rev. Lett. 117, 235302 (2016).

[51] C. Wang, P. Zhang, X. Chen, J. Yu, and H. Zhai, Scheme to Measure the Yopological Number of a Chern Insulator from Quench Dynamics, Phys. Rev. Lett. 118, 185701 (2017).

[52] N. Fläschner, D. Vogel, M. Tarnowski, B. S. Rem, D.-S. Lühmann, M. Heyl, J. C. Budich, L. Mathey, K. Sengstock, and C. Weitenberg, Observation of a dynamical topological phase transition, Nat. Phys. 14, 265 (2018).

[53] W. Sun, C.-R. Yi, B.-Z. Wang, W.-W. Zhang, B. C. Sanders, X.-T. Xu, Z.-Y. Wang, J. Schmiedmayer, Y. Deng, X.-J. Liu, S. Chen, and J.-W. Pan, Uncover Topology by Quantum Quench Dynamics, Phys. Rev. Lett. 121, 250403 (2018).

[54] C. Yang, L. Li, and S. Chen, Dynamical topological invariant after a quantum quench, Phys. Rev. B 97, 060304(R) (2018).

[55] L. Zhang, L. Zhang, S. Niu, and X.-J. Liu, Dynamical classification of topological quantum phases, Sci. Bull. 63, 1385 (2018).

[56] Z. Gong and M. Ueda, Topological Entanglement-Spectrum Crossing in Quench Dynamics, Phys. Rev. Lett. 121, 250601 (2018).

[57] M. McGinley and N. R. Cooper, Topology of One-Dimensional Quantum Systems Out of Equilibrium, Phys. Rev. Lett. 121, 090401 (2018).
[58] M. McGinley and N. R. Cooper, Classification of topological insulators and superconductors out of equilibrium, Phys. Rev. B 99, 075148 (2019).

[59] Y. Xu and Y. Hu, Scheme to equilibrate the Hall response of topological systems from coherent dynamics, Phys. Rev. B 99, 174309 (2019).

[60] M. Tarnowski, F. N. Ünal, N. Fläschner, B. S. Rem, A. Eckardt, K. Sengstock, and C. Weitenberg, Measuring topology by dynamics: Chern number from linking number, Nat. Commun. 10, 1728 (2019).

[61] W. A. Wheeler, L. K. Wagner, and T. L. Hughes, Many-body electric multipole operators in extended systems, Phys. Rev. B 100, 245135 (2019).

[62] B. Kang, K. Shiozaki, and G. Y. Cho, Many-body order parameters for multipoles in solids, Phys. Rev. B 100, 245134 (2019).

[63] S. Ono, L. Trifunovic, and H. Watanabe, Difficulties in operator-based formulation of the bulk quadrupole moment, Phys. Rev. B 100, 245133 (2019).

[64] M. Fruchart, Complex classes of periodically driven topological lattice systems, Phys. Rev. B 93, 115429 (2016).

[65] S. Yao, Z. Yan, and Z. Wang, Topological invariants of floquet systems: General formulation, special properties, and floquet topological defects, Phys. Rev. B 96, 195303 (2017).

[66] B. J. Wieder, Z. Wang, J. Cano, X. Dai, L. M. Schoop, B Bradlyn, and B. A. Bernevig, Strong and "fragile" topological Dirac semimetals with higher-order Fermi arcs, Nat. Commun. 11, 627 (2020).

[67] A. Alexandradinata, X. Dai, and B. A. Bernevig, Wilson-Loop characterization of inversion-symmetric topological insulators, Phys. Rev. B 89, 155114 (2014).

[68] Y. Xu, F. Zhang, and C. Zhang, Structured Weyl Points in Spin-Orbit Coupled Fermionic Superfluids, Phys. Rev. Lett. 115, 265304 (2015).

[69] Y. Xu, Topological gapless matters in three-dimensional ultracold atomic gases, Front. Phys. 14, 43402 (2019).

[70] L. Fidkowski, Entanglement Spectrum of Topological Insulators and Superconductors, Phys. Rev. Lett. 104, 130502 (2010).

[71] M. Aidelsburger, M. Atala, M. Lohse, J. T. Barreiro, B. Paredes, and I. Bloch, Realization of the Hofstadter Hamiltonian with Ultracold Atoms in Optical Lattices, Phys. Rev. Lett. 111, 185301 (2013).

[72] H. Miyake, G. A. Siviloglou, C. J. Kennedy, W. C. Burton, and W. Ketterle, Realizing the Harper Hamiltonian with LaserAssisted Tunneling in Optical Lattices, Phys. Rev. Lett. 111, 185302 (2013).

[73] P. Hauke, M. Lewenstein, and A. Eckardt, Tomography of Band Insulators from Quench Dynamics, Phys. Rev. Lett. 113, 045303 (2014).

[74] N. Fläschner, B. Rem, M. Tarnowski, D. Vogel, D.-S. Lühmann, K. Sengstock, and C. Weitenberg, Exploring the many-body localization transition in two dimensions, Science 352, 1091 (2016).

[75] C. Weitenberg, M. Endres, J. F. Sherson, M. Cheneau, P. Schauß, T. Fukuhara, I. Bloch, and S. Kuhr, Single-spin addressing in an atomic Mott insulator, Nature 471, 319 (2011).

[76] W. S. Bakr, J. Gillen, A. Peng, S. Fölling, and M. Greiner, A quantum gas microscope-Detecting single atoms in a Hubbard regime optical lattice, Nature 462, 74 (2009). 
[77] C. H. Lee, S. Imhof, C. Berger, F. Bayer, J. Brehm, L. W. Molenkamp, T. Kiessling, and R. Thomale, Topolectrical circuits, Commun. Phys. 1, 39 (2018).

[78] T. Hofmann, T. Helbig, C. H. Lee, M. Greiter, and R. Thomale, Chiral Voltage Propagation and Calibration in a Topolectrical Chern Circuit, Phys. Rev. Lett. 122, 247702 (2019).

[79] W.-K. Chen, The Circuits and Filters Handbook, 3rd ed. (CRC Press, Boca Raton, FL, 2009).

[80] H. Li and F. D. M. Haldane, Entanglement Spectrum as a Generalization of Entanglement Entropy: Identification of Topological Order in Non-Abelian Fractional Quantum Hall Effect States, Phys. Rev. Lett. 101, 010504 (2008).
[81] F. Pollmann, A. M. Turner, E. Berg, and M. Oshikawa, Entanglement spectrum of a topological phase in one dimension, Phys. Rev. B 81, 064439 (2010).

[82] I. Peschel, Calculation of reduced density matrices from correlation functions, J. Phys. A 36, L205 (2003).

[83] Y. Lu, N. Jia, L. Su, C. Owens, G. Juzeliūnas, D. I. Schuster, and J. Simon, Probing the Berry curvature and Fermi arcs of a Weyl circuit, Phys. Rev. B 99, 020302(R) (2019).

[84] T. Helbig, T. Hofmann, C. H. Lee, R. Thomale, S. Imhof, L. W. Molenkamp, and T. Kiessling, Band structure engineering and reconstruction in electric circuit networks, Phys. Rev. B 99, 161114(R) (2019). 